\title{
SETS OF PERIODS FOR PIECEWISE MONOTONE TREE MAPS
}

\author{
LL. ALSEDÀ \\ Departament de Matemàtiques, Edifici Cc, Universitat Autònoma de \\ Barcelona, 08913 Cerdanyola del Vallès, Barcelona, Spain \\ On leave at: Departament de Matemàtica Aplicada I, Universitat \\ Politècnica de Catalunya, Diagonal 647, 08028 Barcelona, Spain \\ E-mail: alseda@mat.uab.es \\ D. JUHER \\ Departament d'Informàtica i Matemàtica Aplicada, Universitat de \\ Girona, Lluís Santaló s/n, 17071 Girona, Spain \\ E-mail: david.juher@udg.es \\ P. MUMBRÚ \\ Departament de Matemàtica Aplicada i Anàlisi, Universitat de Barcelona, \\ Gran Via 585, 08071 Barcelona, Spain \\ E-mail: mumbru@mat.ub.es
}

May 29, 2007

\begin{abstract}
We study the set of periods of tree maps $f: T \longrightarrow T$ which are monotone between any two consecutive points of a fixed periodic orbit $P$. This set is characterized in terms of some integers which depend only on the combinatorics of $\left.f\right|_{P}$ and the topological structure of $T$. In particular, a type $p \geq 1$ of $P$ is defined as a generalization of the notion introduced by Baldwin in his characterization of the set of periods of star maps. It follows that there exists a divisor $k$ of the period of $P$ such that if the set of periods of $f$ is not finite then it contains either all the multiples of $k p$ or an initial segment of the $k p \geq$ Baldwin's ordering, except for a finite set which is explicitly bounded. Conversely, examples are given where $f$ has precisely these sets of periods.
\end{abstract}

\section{Introduction}

In this paper we deal with the problem of determining which are the possible sizes of the periodic orbits that appear by iterating a continuous map defined on a tree. For some particular cases (interval and star), several well known results establish that if a continuous map exhibits a periodic orbit which verifies some combinatorial properties then we can determine a set which is a lower bound of the set of periods of the map.

The widely known Sharkovskii's Theorem (see [Sharkovskii, 1964]) studying the set of periods of any continuous map from an interval of the real line into itself was the first remarkable result in this setting. In order to state it, we introduce the Sharkovskii ordering $\unrhd$ (the symbols $\unlhd, \triangleleft$ and $\triangleright$ will be understood in the natural way) in the set $\mathbb{N} \cup\left\{2^{\infty}\right\}$ : 
$3 \unrhd 5 \unrhd 7 \unrhd \ldots \unrhd 2 \cdot 3 \unrhd 2 \cdot 5 \unrhd 2 \cdot 7 \unrhd \ldots \unrhd 4 \cdot 3 \unrhd$ $4 \cdot 5 \unrhd 4 \cdot 7 \unrhd \ldots \unrhd \ldots \unrhd 2^{n} \cdot 3 \unrhd 2^{n} \cdot 5 \unrhd 2^{n} \cdot 7 \unrhd \ldots \unrhd$ $2^{\infty} \unrhd \ldots \unrhd 2^{n} \unrhd \ldots \unrhd 16 \unrhd 8 \unrhd 4 \unrhd 2 \unrhd 1$.

The Sharkovskii's theorem states that if an interval map $f$ has a periodic orbit of period $m$ then $f$ has periodic orbits of period $k$ for each $m \unrhd k$. As a consequence, it can be shown that for each interval map $f$ there exists some $n \in \mathbb{N} \cup\left\{2^{\infty}\right\}$ verifying that the set of periods of $f$ is exactly the set of integers $k$ such that $n \unrhd k$. Conversely, given any $n \in \mathbb{N} \cup\left\{2^{\infty}\right\}$ there exists an interval map $g$ whose set of periods is the set of all integers $k$ such that $n \unrhd k$

During the last three decades there have been several attempts to find results similar to that of Sharkovskii for 1-dimensional spaces other than the interval (see for instance [Alsedà et al., 1989] about maps on $Y$ or [Efremova, 1978], [Block et al., 1980], [Block, 1981] and [Misiurewicz, 1982] about circle maps). More recently, the case of maps defined on trees has been specially treated.

In [Baldwin, 1991] the characterization of the set of periods of any continuous map defined on an $r$-star (a tree with $r$ edges and $r$ endpoints) is given in terms of finitely many partial orderings. Let us define the Baldwin partial orderings ${ }_{p} \geq$ for all $p \in \mathbb{N}$ (the symbols $<_{p}$, $\leq_{p}$ and ${ }_{p}>$ will be understood in the natural way). If $p=1$ then $p \geq$ is the Sharkovskii ordering. For $p>1$ and $k, m \in \mathbb{N} \cup\left\{p 2^{\infty}\right\}$, we write $m_{p} \geq k$ if one of the following cases holds:

(i) $k=1$ or $k=m$

(ii) $k, m \in p \mathbb{N} \cup\left\{p 2^{\infty}\right\}$ and $m / p \triangleright k / p$

(iii) $k \in p \mathbb{N} \cup\left\{p 2^{\infty}\right\}$ and $m \notin\{1\} \cup p \mathbb{N} \cup\left\{p 2^{\infty}\right\}$

(iv) $k, m \notin\{1\} \cup p \mathbb{N} \cup\left\{p 2^{\infty}\right\}$ and $k=i m+j p$ with $i, j \in \mathbb{N}$

where the arithmetic rule $p 2^{\infty} / p=2^{\infty}$ is assumed and $p \mathbb{N}$ stands for $\{p n: n \in \mathbb{N}\}$. It is not difficult to see that $2 \geq$ also coincides with the Sharkovskii ordering.

In Baldwin's paper, a positive integer is associated to each periodic orbit $P$ of an $r$-star map $f$. This integer is called the type of $P$ and depends only on the combinatorics of $\left.f\right|_{P}$ (in Sec. 4 a precise definition is given for a general tree map). Baldwin proves that if $f$ has a periodic orbit of period $m$ and type $p$ then $f$ has periodic orbits of period $k$ for each $m_{p} \geq k$.
An initial segment of the ordering $p \geq$ is defined to be any set $\mathcal{S}$ such that if $m \in \mathcal{S}$ and $m_{p}>k$ then $k \in \mathcal{S}$. Baldwin proves that the set of periods of any $r$-star map is a union of finitely many initial segments of the orderings $p \geq$ for $1 \leq p \leq r$. Conversely, given such a union $\mathcal{A}$ there exists an $r$-star map whose set of periods is $\mathcal{A}$.

In what follows, any continuous map from a tree into itself will be called a tree map.

The characterization of the set of periods for any tree map $f: T \longrightarrow T$ in terms of some constants which depend on the topological structure of $T$ (such as the amount of vertices or endpoints of $T$ ) is yet an open problem. However, there are some partial results in this direction (see, for instance, [Imrich \& Kalinowski, 1985a,b], [Alsedà \& Ye, 1995], [Llibre \& Misiurewicz, 1993] and [Blokh, 1992]).

A natural strategy to obtain this kind of characterization for interval and star maps, that already has been used in the proofs of Sharkovskii and Baldwin theorems, is the following one. Assume that $f$ is an interval map or an $r$-star map and let $P$ be a periodic orbit of $f$. The first stage of the strategy consists of studying the subset $\Lambda_{P}$ of periods of $f$ which are forced by the pattern of $P$. That is, one wants to know which other orbits the map $f$ will necessarily have, depending only on the combinatorics of $\left.f\right|_{P}$. To solve this problem one replaces $f$ by another map $g$ such that $\left.g\right|_{P}=\left.f\right|_{P}$ and $g$ is monotone between any two consecutive points of $P$. It can be seen that such a map is the dynamically simplest model which exhibits an orbit having the pattern of $P$. This means that each pattern exhibited by $g$ is also exhibited by $f$ and that the set $\Lambda_{P}$ coincides with the set of periods of $g$. Therefore, the set $\Lambda_{P}$ can be computed just by studying the loops of the Markov graph of $g$. The last step of the proof consists in considering each orbit $P$ of $f$ and its associated $\Lambda_{P}$. Then one gets the structure of the set of periods of $f$ by obtaining the structure of the (uncountable) union of all sets $\Lambda_{P}$. This is done by purely number-theoretical arguments.

As it has been said before, an important intermediate step in getting the periodic structure of interval and star maps is the study of the set of periods of these (piecewise monotone) "dynamically simplest models". Since, in addition, piecewise monotone maps provide all the necessary ex- 
amples in the "converse part" of the theorems of Sharkovskii and Baldwin, the proofs of these results are strongly based on the study of this class of maps.

To study the set of periods of tree maps we have chosen to follow a strategy similar to the one described above (as we shall see, this is a natural strategy also in the case of tree maps). However, it turns out that the straightforward implementation of this strategy to tree maps does not work. Indeed, let $f: T \longrightarrow T$ be a tree map, let $P$ be a periodic orbit of $f$ and let $V$ denote the set of vertices of $T$. Then we want to consider a $P$-weakly monotone map $g$ which is defined to coincide with $f$ on $V \cup P$ and is monotone (injective) on the closure of each connected component of $T \backslash(V \cup P)$. The problem is that a $P$-weakly monotone map can have (even infinitely many) periods which are not periods of $f$, and thus it cannot be our desired "minimal model". To illustrate this phenomenon consider the following simple example in the case of interval maps.

Example 1.1. Let $g:[0,1] \longrightarrow[0,1]$ denote the tent map such that the point $1 / 2$ is a periodic point of period 3. That is:

$$
g(x)= \begin{cases}\mu x & \text { when } x \in[0,1 / 2], \\ \mu(1-x) & \text { when } x \in[1 / 2,1],\end{cases}
$$

with $\mu=\frac{1+\sqrt{5}}{2}$. This map has periodic points of all periods. Set $p=g(1 / 2)=\frac{1+\sqrt{5}}{4}$ and let $f:[0,1] \longrightarrow[0,1]$ be the continuous map such that $f(0)=f(1)=0, f(x)=p$ for each $x \in[1-p, p]$ and $f$ is affine on $[0,1-p]$ and $[p, 1]$. Clearly, $p$ is a fixed point of $f$ and 1 is the only period of $f$. Now consider $T=[0,1]$ as a 2 -star with vertices $V=\{0,1 / 2,1\}$ and suppose that we are given the map $f$ with $P=\{0\}$. The map $g$ coincides with $f$ on $V \cup P$ and is monotone (injective) on the closure of each connected component of $T \backslash(V \cup P)$ (so, $g$ is $P$-weakly monotone). However the map $g$ has periodic points of all periods whereas the map $f$ only has fixed points.

The above example tells us that it is not straightforward to extend the notion of "minimal model" (or "P-minimal map") to the setting of tree maps. However, in [Alsedà et al., 1997] the authors give a definition of pattern of $P$ and prove that there always exists a tree $S_{P}$ and a map $g_{P}: S_{P} \longrightarrow S_{P}$ exhibiting a periodic orbit $Q$ with the same pattern as $P$ and displaying dynamic minimality properties similar to the known ones for the interval case. The crucial point is that the map $g_{P}$ is $Q$-monotone which means that it is monotone between any two consecutive points of $Q$ (two points $a, b$ of $Q$ are said to be consecutive if there are no other points of $Q$ in the convex hull of $\{a, b\})$. We also remark that the tree $S_{P}$, which may be different from $T$, is unique up to homeomorphisms and collapse of invariant forests. The map $g_{P}$, which is the crucial tool in our strategy, is called a P-minimal model. As an example consider the maps $f$ and $g$ defined in Fig. 1: It turns out that the orbits $P$ and $Q$ have the same pattern (even living in two different trees) and that the map $g$ is the minimal model corresponding to this pattern. Observe also that the notion of $Q$ monotonicity is stronger than the notion of $Q$-weak monotonicity. To see it, consider the map $f$ defined in Fig. 1 and observe that there does not exist any $P$-monotone map $\varphi: T \longrightarrow T$ which coincides with $f$ on the set $P$. Such a map $\varphi$ would have to satisfy $\varphi\left(\left[x_{1}, x_{2}\right]\right)=\left[x_{2}, x_{3}\right]$ and $\varphi\left(\left[x_{3}, x_{5}\right]\right)=\left[x_{4}, x_{6}\right]$. Thus $\varphi(z) \in\left[x_{2}, x_{3}\right] \cap\left[x_{4}, x_{6}\right]$; a contradiction.

Now we are ready to describe the implementation of the strategy we use to study the set of periods of tree maps:

(1) For each periodic orbit $P$ of $f$ calculate $\Lambda_{P}$, the set of periods of the corresponding $P$ minimal model $g_{P}: S_{P} \longrightarrow S_{P}$ or, if this is not possible, estimate the largest possible subset of $\Lambda_{P}$.

(2) Prove that the set of periods of the $P$-minimal model $g_{P}$ is contained in the set of periods of each tree map which exhibits an orbit with the pattern of $P$. In particular, $\Lambda_{P}$ is a subset of the set of periods of $f$.

(3) Consider each orbit $P$ of $f$ and its associated $\Lambda_{P}$. Then one can obtain the structure of the set of periods of $f$ by describing the structure of the (uncountable) union of all sets $\Lambda_{P}$.

The main result of this paper performs step (1) of the above program by means of the study of the Markov graph of $g_{P}$. Indeed, given any tree map $g: S \longrightarrow S$ having a periodic orbit $Q$ and such that $g$ is $Q$-monotone, we use information from the combinatorics of $\left.g\right|_{Q}$ and the topological structure of $S$ in order to study the Markov graph of $g$ and compute as large as possible subsets of the set of 

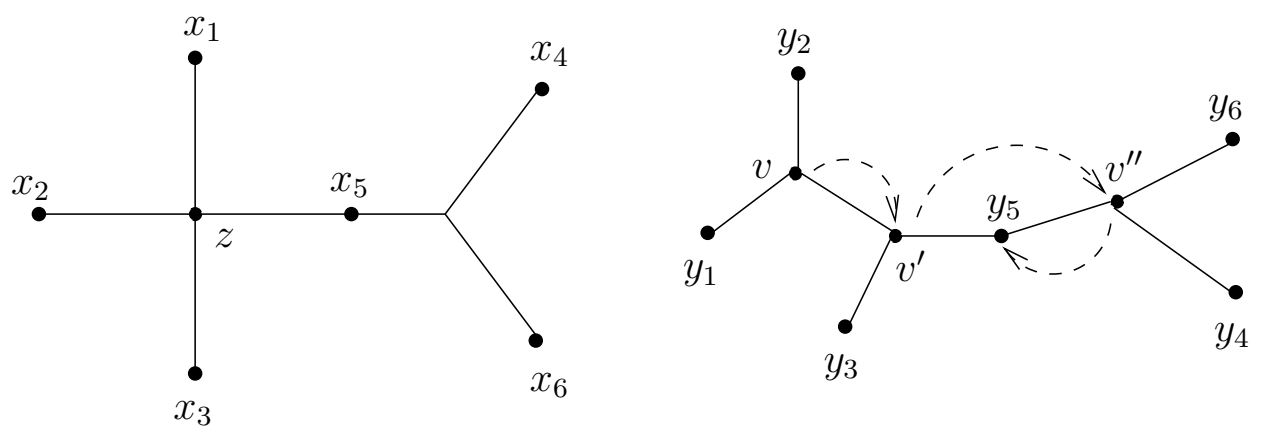

Fig. 1. Left figure: A tree $T$ and a map $f: T \longrightarrow T$ which exhibits an orbit $P=\left\{x_{1}, x_{2}, \ldots, x_{6}\right\}$ with $f\left(x_{i}\right)=x_{i+1}$ for $1 \leq i \leq 5$ and $f\left(x_{6}\right)=x_{1}$. This map can be made $P$-weakly monotone by setting $f(z) \in P \cup\{z\}$ but it cannot be made $P$-monotone.

Right figure: A tree $S$ and a map $g: S \longrightarrow S$ having an orbit $Q=\left\{y_{1}, y_{2}, \ldots, y_{6}\right\}$ with $g\left(y_{i}\right)=y_{i+1}$ for $1 \leq i \leq 5$ and $g\left(y_{6}\right)=y_{1}$. If in addition we take $g(v)=v^{\prime}, g\left(v^{\prime}\right)=v^{\prime \prime}$ and $g\left(v^{\prime \prime}\right)=y_{5}$ then $g$ can be made $Q$-monotone (and thus $Q$-weakly monotone).

periods of $g$. Moreover, examples are given where the difference between the whole set of periods and these subsets is finite and explicitly bounded.

Since in general $T$ and $S_{P}$ differ (unless $T$ is an interval or a star) it is not easy to carry out steps (2) and (3) of the above program. These two steps will be matter of a forthcoming paper by the same authors.

\section{Basic Definitions and Statement of the Main Results}

Let $X$ be a topological space and let $f: X \longrightarrow X$ be a map. As usual, $f^{0}=\mathrm{Id}$ and $f^{k}=f \circ f \circ \cdots \circ f$ ( $k$ times) for $k \in \mathbb{N}$. For a finite set $A$ we will denote its cardinality by $|A|$. Given a point $x \in X$ we define its orbit, denoted by $\operatorname{Orb}_{f}(x)$ (or simply by $\operatorname{Orb}(x))$, to be the set $\left\{f^{k}(x): k=0,1,2, \ldots\right\}$. If $|\operatorname{Orb}(x)|=n$, then $f^{k}(x) \neq x$ for $0<k<n$ and $f^{n}(x)=x$. In this case we say that $x$ is a periodic point of $f$ of period $n$ (or an $n$-periodic point of $f$ ) and that $\operatorname{Orb}_{f}(x)$ is a periodic orbit of $f$ of period $n$ (or an n-periodic orbit of $f$ ). A point of period 1 is called a fixed point, and the set of fixed points of $f$ will be denoted by $\operatorname{Fix}(f)$. The set of periods of $f$, denoted by $\operatorname{Per}(f)$, is the set of periods of all periodic orbits of $f$. Given a point $x \in X$, we say that $x$ is eventually periodic if it is not periodic but $f^{n}(x)$ is periodic for some $n>0$. If $A \subset \mathbb{N}$ and $m, n \in \mathbb{N}, n A$ stands for $\{n k: k \in A\}$ and $m+n A$ stands for $\{m+n k: k \in A\}$.

A tree is a compact uniquely arcwise connected space which is a point or a union of a finite number of intervals (from now on, by an interval we mean any space homeomorphic to $[0,1])$. Any continuous map from a tree into itself will be called a tree map. If $T$ is a tree and $x \in T$, we define the valence of $x$ to be the number of connected components of $T \backslash\{x\}$. Each point of valence 1 will be called an endpoint of $T$ and the set of such points will be denoted by $\operatorname{En}(T)$. Each point of valence different from 2 will be called a vertex of $T$ and the set of vertices of $T$ will be denoted by $V(T)$. As usual, the closure of each connected component of $T \backslash V(T)$ will be called an edge of $T$. Any tree which is a union of $r>1$ intervals whose intersection is a unique point $x$ of valence $r$ will be called an $r$-star, and $x$ will be called the central point.

If $X$ is a topological space and $f: X \longrightarrow X$ is a map, we will say that a set $A \subset X$ is $f$-invariant if $f(A) \subset A$. For a set $B \subset X$ we will denote by $\operatorname{Int}(B)$ and $\mathrm{Cl}(B)$ the interior and the closure of $B$ respectively. Let $S$ be a tree. Given $P \subset S$ we will define the convex hull of $P$, denoted by $\langle P\rangle_{S}$ or simply by $\langle P\rangle$, as the smallest closed connected subset of $S$ containing $P$. When $P=\{x, y\}$ we will write $\langle x, y\rangle$ or $[x, y]$ to denote $\langle P\rangle$. The notations $(a, b)$, $(a, b]$ and $[a, b)$ will be understood in the natural way.

Let $g: S \longrightarrow S$ be a tree map. Given $a, b \in S$ we say that $\left.g\right|_{[a, b]}$ is monotone if either $g([a, b])$ is a point or it is an interval and, given two homeomorphisms $\phi:[0,1] \longrightarrow[a, b]$ and $\varphi: g([a, b]) \longrightarrow[0,1]$, then $\varphi \circ g \circ \phi:[0,1] \longrightarrow[0,1]$ is monotone (as a real 
function). If $P \subset S$ is a finite $g$-invariant set which contains $\operatorname{En}(S)$, we say that $g$ is $P$-monotone if $g([a, b])=[g(a), g(b)]$ and $\left.g\right|_{[a, b]}$ is monotone whenever $[a, b] \cap P=\{a, b\}$. In this case we will say that the triplet $(S, P, g)$ is a monotone model. If in addition $P$ contains a unique periodic orbit and this orbit consists of a fixed point, then we will say that $(S, P, g)$ is a trivial model. Observe that if $(S, P, g)$ is a trivial monotone model and $P$ consists of a fixed point then $S$ reduces to the unique point of $P$ since $\operatorname{En}(S) \subset P$.

Remark 2.1. If $(S, P, g)$ is a monotone model, it is shown in Proposition 4.2 of [Alsedà et al., 1997] that the image of each vertex $z$ is uniquely determined and is either a vertex or belongs to $P$. In fact, if $a, b, c \in P$ in such a way that $z \in[a, b] \cap[a, c] \cap[b, c]$ and $\langle\{a, b, c\}\rangle_{S} \backslash P$ is connected, then it can be easily seen that $g(z)$ is the only point contained in $g([a, b]) \cap g([a, c]) \cap g([b, c])$.

Let $(S, P, g)$ be a monotone model and let $Q=$ $P \cup V(T)$. Observe that each connected component of $T \backslash Q$ is an interval. By Remark 2.1, $Q$ is $g$-invariant. It is not difficult to see that $g$ is monotone on each connected component of $T \backslash Q$. In this situation, we can consider the usual notion of the Markov graph of $g$, whose vertices are closures of connected components of $T \backslash Q$ and there is an arrow from $K$ to $L$ if and only if $g(K) \supset L$. It is folk knowledge that there is a certain correspondence between periodic orbits of $g$ and loops of its Markov graph (see Sec. 3).

Now we informally sketch the strategy that we use in this paper in order to calculate the set of periods of a monotone model. Let $(S, P, g)$ be a non-trivial monotone model such that $P$ is a periodic orbit of $g$. The basic tool we use to obtain periodic points of $g$ is the existence of a special kind of loops in the Markov graph of $g$, which we call $e x$ ternal loops (see Sec. 4). The set of external loops in the Markov graph of $g$ which in addition verify certain technical properties will be denoted by $\tilde{\mathcal{E}}(S, P, g)$. If $\tilde{\mathcal{E}}(S, P, g) \neq \emptyset$ then $\operatorname{Per}(g)$ is directly calculable (see Lemma 4.6 and Theorem 4.7).

If $\tilde{\mathcal{E}}(S, P, g)=\emptyset$ then we proceed as follows. Set $\left(S_{1}, P_{1}, g_{1}\right)=(S, P, g)$. We prove that there exist $p_{1} \in \mathbb{N}$ and a monotone model $\left(S_{2}, P_{2}, g_{2}\right)$ such that $S_{2} \subset S_{1}, g_{2}=\left.g_{1}^{p_{1}}\right|_{S_{2}}$ and $\operatorname{Per}\left(g_{1}\right) \supset p_{1} \operatorname{Per}\left(g_{2}\right)$.
Such a monotone model is called a partial $p_{1}$ reduction of $\left(S_{1}, P_{1}, g_{1}\right)$. If we are able to compute $\operatorname{Per}\left(g_{2}\right)$, then the estimation $p_{1} \operatorname{Per}\left(g_{2}\right)$ for the set of periods of $g_{1}$ is optimal, since we know examples verifying $\operatorname{Per}\left(g_{1}\right)=p_{1} \operatorname{Per}\left(g_{2}\right)$. So the problem of estimating $\operatorname{Per}\left(g_{1}\right)$ is reduced to compute $\operatorname{Per}\left(g_{2}\right)$. If $\tilde{\mathcal{E}}\left(S_{2}, P_{2}, g_{2}\right)=\emptyset$, we can iterate this procedure. In Sec. 6 it is shown that we can proceed in this way as many times as necessary in order to obtain a finite sequence of monotone models $\left\{\left(S_{i}, P_{i}, g_{i}\right)\right\}_{i=1}^{m}$ such that:

(i) $\left(S_{1}, P_{1}, g_{1}\right)=(S, P, g)$.

(ii) $\left(S_{i+1}, P_{i+1}, g_{i+1}\right)$ is a $p_{i}$-partial reduction of $\left(S_{i}, P_{i}, g_{i}\right)$ for $1 \leq i<m$.

(iii) $P_{i}$ contains a unique periodic orbit $P_{i}^{\circ}$ and $\left|P_{i}^{\circ}\right|=p_{i}\left|P_{i+1}^{\circ}\right|$ for $1 \leq i<m$. Moreover, $P_{i}^{\circ} \subset P_{i+1} \varsubsetneqq P_{i}$ when $p_{i}=1$.

(iv) $\tilde{\mathcal{E}}\left(S_{i}, P_{i}, g_{i}\right)=\emptyset$ for $1 \leq i<m$.

(v) Either $\left(S_{m}, P_{m}, g_{m}\right)$ is a trivial model or it verifies $\tilde{\mathcal{E}}\left(S_{m}, P_{m}, g_{m}\right) \neq \emptyset$.

Since $\operatorname{Per}\left(g_{i}\right) \supset\{1\} \cup p_{i} \operatorname{Per}\left(g_{i+1}\right)$, we can easily get that $\operatorname{Per}(g) \supset\left\{1, p_{1}, p_{1} p_{2}, \ldots, p_{1} p_{2} \cdots p_{m-1}\right\} \cup$ $p_{1} p_{2} \cdots p_{m-1} \operatorname{Per}\left(g_{m}\right)$. Furthermore, since $P=$ $P_{1}=P_{1}^{\circ}$, we have that $|P|=p_{1} p_{2} \cdots p_{m-1}\left|P_{m}^{\circ}\right|$. We remark that such a sequence of partial reductions of $(S, P, g)$ is not unique.

By means of the above construction, a complete reduction of $(S, P, g)$ is defined to be the pair $\{\mathcal{R}, K\}$ where $K=\left\{1, p_{1}, p_{1} p_{2}, \ldots, p_{1} p_{2} \cdots p_{m-1}\right\}$ and $\mathcal{R}=\left(S_{m}, P_{m}, g_{m}\right)$. Note that if $\tilde{\mathcal{E}}(S, P, g) \neq \emptyset$ then $m=1$ and thus $K$ reduces to $\{1\}$. The model $\mathcal{R}$ will be called a completely reduced model of $(S, P, g)$. It satisfies:

(i) $g_{m}=\left.g^{\max K}\right|_{S_{m}}$.

(ii) $P_{m}$ contains a unique periodic orbit $P_{m}{ }^{\circ}$ and $|P|=\left|P_{m}{ }^{\circ}\right| \cdot \max K$.

(iii) $\operatorname{Per}(g) \supset K \cup(\max K) \cdot \operatorname{Per}\left(g_{m}\right)$.

Since there exist many sequences of partial reductions, a complete reduction of $(S, P, g)$ is not uniquely determined.

By (iii), the study of the set of periods of a monotone model can be reduced to the study of the set of periods of its completely reduced models. This is the strategy we use in this paper and it gives rise to our main result. In order to state it, we need to introduce some more notation.

Let $\mathcal{R}=(\bar{S}, \bar{P}, \bar{g})$ be a non-trivial completely reduced model of a given monotone model $(S, P, g)$. 
We will prove that $\operatorname{Per}(\bar{g})$ depends on three nonnegative constants (besides $\left|\bar{P}^{\circ}\right|$, of course). These constants can be directly calculated from the combinatorics induced by $\bar{g}$ on the $\bar{g}$-invariant set $\bar{P} \cup$ $V(\bar{S})$. Since these numbers strongly depend on the topological structure of the tree $\bar{S}$ and the behavior of $\bar{g}$ on $\bar{P}$, we denote them by $n(\mathcal{R}), p(\mathcal{R})$ and $q(\mathcal{R})$ in order to stress their dependence from the model. The constant $n(\mathcal{R})$ is the minimum integer $n$ such that $\bar{g}^{n}(\bar{P})=\bar{P}^{\circ}$. On the other hand, $p(\mathcal{R})$ is called a type of the model, and essentially is a generalization of the notion of type of a periodic orbit introduced in [Baldwin, 1991] for star maps. Finally $q(\mathcal{R})$ will be called the rotation index of the model. The precise definition of these constants is given in Sec. 4.

Next we introduce a notation to deal with a special type of initial segments of the $p \geq$ orderings. If $p \in \mathbb{N}$ and $r \in \mathbb{N} \cup\left\{p 2^{\infty}\right\}$, we define $\mathcal{S}_{p}(r)=$ $\left\{k \in \mathbb{N}: r_{p} \geq k\right\}$. Note that if $r \in p \mathbb{N}$ then $\mathcal{S}_{p}(r)=\{1\} \cup p\{k \in \mathbb{N}: r / p \unrhd k\}$ and if $r \notin p \mathbb{N}$ then $\mathcal{S}_{p}(r)=\{1, r\} \cup\{r i+p j: i \geq 0, j \geq 1\}$. Given $p, r \in \mathbb{N}$, we define

$$
\mathcal{S}_{p}^{*}(r)= \begin{cases}\mathcal{S}_{p}(r) & \text { if } r \notin p \mathbb{N} \\ \mathcal{S}_{p}(3 p) & \text { if } r \in p \mathbb{N}\end{cases}
$$

Observe that if $r \in p \mathbb{N}$ then $\mathcal{S}_{p}^{*}(r)=p \mathbb{N} \cup\{1\} \supset$ $\mathcal{S}_{p}(r)$.

Remark 2.2. Let $k, p, r$ be natural numbers. Then we have that $\{1\} \cup k \mathcal{S}_{p}^{*}(r)=\{k\} \cup \mathcal{S}_{k p}^{*}(k r)$. Indeed, if $r \notin p \mathbb{N}$ then $\{1\} \cup k \mathcal{S}_{p}^{*}(r)=\{1\} \cup k(\{1, r\} \cup\{r i+$ $p j: i \geq 0, j \geq 1\})=\{1, k, k r\} \cup\{k r i+k p j: i \geq$ $0, j \geq 1\}=\{k\} \cup \mathcal{S}_{k p}^{*}(k r)$. On the other hand, when $r \in p \mathbb{N}$ we get $\{1\} \cup k \mathcal{S}_{p}^{*}(r)=\{1\} \cup k(p \mathbb{N} \cup\{1\})=$ $\{1, k\} \cup k p \mathbb{N}=\{k\} \cup \mathcal{S}_{k p}^{*}(k r)$.

From now on, we take $\{1,2, \ldots, n\}$ as the representatives of the classes of $\mathbb{Z} / n \mathbb{Z}$.

Now we are ready to state the main results of this paper.

Theorem A. Let $(S, P, g)$ be a monotone model such that $P$ is a periodic orbit of $g$. If $P$ consists of a fixed point of $g$ then $\operatorname{Per}(g)=\{1\}$. Otherwise, there exist complete reductions of $(S, P, g)$. For any complete reduction $\{\mathcal{R}, K\}$ of $(S, P, g)$, we have that $\operatorname{Per}(g) \supset K$. If, in addition, $\mathcal{R}$ is non-trivial and we denote $p(\mathcal{R}), q(\mathcal{R}), n(\mathcal{R})$ and $\max K$ by $p, q, n$ and $k$ respectively, then

$$
\operatorname{Per}(g) \supset K \cup \mathcal{S}_{k p}^{*}(|P|+l k p) \backslash\{2 k p, 3 k p, \ldots, \lambda k p\}
$$

for some $0 \leq \lambda p \leq \frac{|P|}{k}+p+q+n+1$ and some $0 \leq l \leq \frac{|P|}{k}+q+1$. Furthermore, if $n=0$ then $l p \leq p+q-(q \bmod p)$.

The periods computed in the proof of Theorem A correspond to periodic orbits which do not intersect the set $V(S)$ of vertices of $S$. We additionally prove (see Corollary 6.8 ) that $\operatorname{Per}(g)$ contains a finite set $\mathcal{V}$ whose elements divide the least common multiple of the periods of all periodic orbits contained in $V(S)$.

Remark 2.3. When $|P| \in k p \mathbb{N}$ the upper bound for $l$ in Theorem A is irrelevant, since $\mathcal{S}_{k p}^{*}(|P|+l k p)=$ $k p \mathbb{N}$ for any $l$. On the other hand, when $|P| \notin k p \mathbb{N}$ the upper bound for $l$ controls how far $\mathcal{S}_{k p}^{*}(|P|+l k p)$ is from $\mathcal{S}_{k p}^{*}(|P|)$. Indeed, it is easy to check that $\mathcal{S}_{k p}^{*}(|P|) \backslash \mathcal{S}_{k p}^{*}(|P|+l k p)=\{i|P|+j k p: 1 \leq i<$ $k p, 1 \leq j \leq i l\}$.

Sometimes a continuous self-map of a compact space is called chaotic if it has positive topological entropy (see [Denker et al., 1976] for a definition). Then it can be derived from Theorem $\mathrm{E}$ of [Llibre \& Misiurewicz, 1993] and Theorem A that if $\mathcal{R}$ is a non-trivial model then $g$ is chaotic. And conversely, it is not difficult to see that if $g$ is not chaotic then $\operatorname{Per}(g)$ must be finite (this is true only for monotone models). Thus the monotone models with a trivial (respectively non-trivial) completely reduced model correspond to zero entropy (resp. chaotic) maps.

We must stress the fact that there are some known results which describe the set of periods of some kinds of tree maps except for a finite set of periods (see for instance [Blokh, 1991] and [Alsedà \& Ye, 1995]). Nevertheless, nothing is said usually about this finite set. Theorem A states that the set of periods of a (chaotic) monotone model contains a set $\mathcal{C}$ which is $\mathcal{S}_{k p}^{*}(|P|)$ except for an explicitly bounded finite set of periods. In fact, from Remark 2.3 it follows that $\mathcal{S}_{k p}^{*}(|P|) \backslash \mathcal{C}$ is exactly $\{2 k p, 3 k p, \ldots, \lambda k p\}$ if $|P| \in k p \mathbb{N}$ and $\{2 k p, 3 k p, \ldots, \lambda k p\} \cup\{i|P|+j k p: 1 \leq i<k p, 1 \leq$ $j \leq i l\}$ otherwise. Thus the difference between $\mathcal{C}$ and $\mathcal{S}_{k p}^{*}(|P|)$ depends on the constants $\lambda$ and 
$l$, which depend on combinatorial data extracted from the model by means of the constants $q$ and $n$. The smaller $q$ and $n$ are, the bigger (and closer to $\left.\mathcal{S}_{k p}^{*}(|P|)\right) \mathcal{C}$ is.

A natural question arises: how accurate is the estimation of $\operatorname{Per}(g)$ given by Theorem A in relation to Sharkovskii and Baldwin theorems when $S$ is an interval or a star? Given $r \in \mathbb{N}$, let us write $\mathrm{Sh}(r)$ for the initial segment of Sharkovskii's ordering starting at $r$. That is, $\operatorname{Sh}(r)=\{s \in \mathbb{N}: r \unrhd s\}$.

Suppose that $S$ is an interval and $|P|=t \cdot 2^{s}$ with $t$ odd and $s>1$. Assume in addition that $P$ has no division (see for instance [Li et al., 1982]). Then from the proof of Theorem A one gets that $(S, P, g)$ admits a complete reduction $\{\mathcal{R}, K\}$ with $\mathcal{R}=(S, P, g), K=\{1\}, q=n=l=0$ and $p \in\{1,2\}$. If $p=1$ then Theorem A states that $\operatorname{Per}(g) \supset \mathbb{N} \backslash\{2,3, \ldots, \lambda\}$. When $p=2$, we get $\operatorname{Per}(g) \supset 2 \mathbb{N} \backslash\{4,6, \ldots, 2 \lambda\}$. In both cases, these sets contain infinitely many periods which are not in $\operatorname{Sh}\left(t \cdot 2^{s}\right)$. Theorem A can provide more information than Sharkovskii's theorem, since in our result other combinatorial features of the orbit $P$, besides its period, are taken into account. This goes in the direction of the main result of [Li et al., 1982], Baldwin's theorem and other several results in the same spirit (see [Misiurewicz \& Nitecki, 1991] or [Alsedà \& Ye, 1995]).

Assume that $S$ is an interval and $P$ is a primary orbit (see [Baldwin, 1987] or [Alsedà et al., 1989]) of period $t \cdot 2^{s}$ with $t$ odd and $s \geq 0$. Then $\operatorname{Per}(g)=$ $\operatorname{Sh}\left(t \cdot 2^{s}\right)$, and it is not difficult to see that $(S, P, g)$ admits a complete reduction $\{\mathcal{R}, K\}$ such that $K=$ $\left\{1,2,2^{2}, \ldots, 2^{s}\right\}$ and $\mathcal{R}$ is a trivial model if and only if $t=1$. If $\mathcal{R}$ is trivial then Theorem A states that $\operatorname{Per}(g) \supset K=\left\{1,2,2^{2}, \ldots, 2^{s}\right\}=\operatorname{Sh}\left(2^{s}\right)$. On the other hand, when $\mathcal{R}$ is not trivial from the proof of Theorem A one gets that $q=n=l=0, k=2^{s}$, $p=2$ and $\lambda=\frac{t-1}{2}$. Hence Theorem A states that

$$
\begin{aligned}
\operatorname{Per}(g) \supset & K \cup \mathcal{S}_{2^{s+1}}^{*}\left(t \cdot 2^{s}\right) \backslash \\
& \left\{2 \cdot 2^{s+1}, 3 \cdot 2^{s+1}, \ldots, \frac{t-1}{2} \cdot 2^{s+1}\right\} \\
= & \left\{1,2,2^{2}, \ldots, 2^{s}\right\} \cup\left\{t \cdot 2^{s}\right\} \cup \\
& 2^{s}\{t i+2 j, i \geq 0, j \geq 1\} \backslash \\
& \left\{2 \cdot 2^{s+1}, 3 \cdot 2^{s+1}, \ldots, \frac{t-1}{2} \cdot 2^{s+1}\right\} .
\end{aligned}
$$

It is not difficult to show that this set is exactly $\operatorname{Sh}\left(t \cdot 2^{s}\right) \backslash\left\{2 \cdot 2^{s+1}, 3 \cdot 2^{s+1}, \ldots, \frac{t-1}{2} \cdot 2^{s+1}\right\}$.
A similar calculus can be done when $S$ is an $r$ star (with $r \geq 3$ ) and $P$ is a primary orbit. Some of these computations are shown in Table 1. When $|P| \notin r \mathbb{N}$ then $g$ is the $(|P|, r)$-spiral map (see [Baldwin, 1991]).

Thus, when $S$ is an interval or a star, in some cases Theorem A misses out the subset of periods $\{2 k p, 3 k p, \ldots, \lambda k p\}$. Nevertheless, in these cases it can be shown that $g^{k p}$ exhibits a horseshoe. Then it easily follows that $\operatorname{Per}(g) \supset k p \mathbb{N}$. In particular, $\operatorname{Per}(g) \supset\{2 k p, 3 k p, \ldots, \lambda k p\}$. The existence of this horseshoe is due to the (geometric) fact that there are no vertices of $S$ between consecutive points of $P$. For a general tree map it is not true that $g^{k p}$ has a horseshoe, and thus $\operatorname{Per}(g)$ does not necessarily contain $k p \mathbb{N}$.

Also the following natural question arises: do there exist monotone models whose set of periods contains exactly the periods of Theorem A and no other? Before answering this question, we must give the range of possible values of the constants $p, q, n$ and $k$ in Theorem A. We have that $p \geq 1, q \geq 0$ and $n \in\{0,1,2\}$. Set $r=|P| / k$. In Corollary 8.2 we show that the values of $p$ and $q$ are bounded in terms of $r$. In particular, when $n=0$ we have that

$$
\begin{gathered}
p \leq r-1, \\
q+4 \leq r \text { when } p=1 \text { and } \\
2 p+q+1 \leq r \text { when } q>0 .
\end{gathered}
$$

The answer to the above question is given by the following converse of Theorem A:

Theorem B. Let $K \subset \mathbb{N}$ be a set of the form $\left\{1, k_{1}, k_{2}, \ldots, k_{m}\right\}$ such that $k_{1}>1$ and $k_{i}$ strictly divides $k_{i+1}$ for $1 \leq i<m$. Set $k=k_{m}$. Then:

(a) There exists a monotone model $(R, B, h)$ such that $|B|=k$ and $\operatorname{Per}(h)=K$.

(b) Given any $r>1, p \geq 1$ and $q \geq 0$ verifying (1), there exists a monotone model $(S, P, g)$ and a complete reduction $\{(\bar{S}, \bar{P}, \bar{g}), K\}$ of $(S, P, g)$ such that $\left|\bar{P}^{\circ}\right|=r, p(\bar{S}, \bar{P}, \bar{g})=p$, $q(\bar{S}, \bar{P}, \bar{g})=q, n(\bar{S}, \bar{P}, \bar{g})=0$ and $\operatorname{Per}(g)=$ $K \cup \mathcal{C}$, where $\mathcal{C}$ is a set such that

$$
\begin{aligned}
\mathcal{S}_{k p}^{*}(|P|+l k p) \backslash\{2 k p, 3 k p, \ldots, \lambda k p\} \\
\subset \mathcal{C} \subset \mathcal{S}_{k p}^{*}(|P|)
\end{aligned}
$$

with $l p=p+q-(q \bmod p)$ and $\lambda p$ being the largest multiple of $p$ smaller than $r+p+q+1$. 
Table 1. Some examples of sets of periods given by Theorem A and Theorems of Sharkovskii and Baldwin.

\begin{tabular}{|c|c|c|c|}
\hline Model & Complete reduction & $\begin{array}{l}\text { Sharkovskii's or } \\
\text { Baldwin's Theorem }\end{array}$ & Theorem A \\
\hline $\begin{array}{l}S \text { interval, } \\
|P|=t \cdot 2^{s} \\
t \text { odd, } s>1 \\
\text { no division }\end{array}$ & $\begin{array}{l}\mathcal{R}=(S, P, g) \\
K=\{1\} \\
q=n=l=0, p \in\{1,2\}\end{array}$ & $\operatorname{Sh}\left(t \cdot 2^{s}\right)$ & $p \mathbb{N} \backslash\{2 p, 3 p, \ldots, \lambda p\}$ \\
\hline $\begin{array}{l}S r \text {-star, } \\
|P|=r \cdot 2^{s} \\
P \text { primary }\end{array}$ & $\begin{array}{l}\mathcal{R} \text { trivial, } \\
K=\left\{1, r, 2 r, 2^{2} r, \ldots, 2^{s} r\right\}\end{array}$ & $\mathcal{S}_{r}\left(r \cdot 2^{s}\right)$ & $\mathcal{S}_{r}\left(r \cdot 2^{s}\right)$ \\
\hline $\begin{array}{l}S r \text {-star, } \\
|P|=r t \cdot 2^{s} \\
t>1 \text { odd } \\
P \text { primary }\end{array}$ & $\begin{array}{l}\mathcal{R} \text { non-trivial, } \\
K=\left\{1, r, 2 r, 2^{2} r, \ldots, 2^{s} r\right\} \\
q=n=l=0, p=2 \\
k=2^{s} r, \lambda=\frac{t-1}{2}\end{array}$ & $\mathcal{S}_{r}\left(r t \cdot 2^{s}\right)$ & $\begin{array}{c}\mathcal{S}_{r}\left(r t \cdot 2^{s}\right) \backslash r \cdot\left\{2 \cdot 2^{s+1},\right. \\
\left.3 \cdot 2^{s+1}, \ldots, \frac{t-1}{2} \cdot 2^{s+1}\right\}\end{array}$ \\
\hline $\begin{array}{l}S r \text {-star, } \\
|P|=s \notin r \mathbb{N} \\
(s, r) \text {-spiral map }\end{array}$ & $\begin{array}{l}\mathcal{R} \text { non-trivial, } \\
K=\{1\}, q=n=l=0, \\
p=r, \lambda=\frac{s-(s \bmod r)}{r}\end{array}$ & $\mathcal{S}_{r}(s)$ & $\begin{array}{c}\mathcal{S}_{r}(s) \backslash \\
\{2 r, 3 r, \ldots, s-(s \bmod r)\}\end{array}$ \\
\hline
\end{tabular}

In order to simplify the proof of Theorem B, we have considered only models for which $n=0$. In fact, according to Theorem $\mathrm{A}$, if one looks for a characterization of $\operatorname{Per}(g)$ up to a finite set then the values of $q$ and $n$ are irrelevant.

This paper is organized as follows. In Sec. 3 we introduce the usual $f$-covering tools which relate the periodic orbits of a map and the loops of its associated Markov graph. In Sec. 4 we define a particular class of monotone models, which we call $y$-expansive, and we compute periodic orbits associated to the loops of the Markov graph of $y$ expansive models. In Sec. 5 we use the notion of a canonical model introduced in [Alsedà et al., 1997]. From each monotone model $(S, P, g)$ we construct a canonical model $\left(S^{\prime}, P^{\prime}, g^{\prime}\right)$ and find a relation between $\operatorname{Per}(g)$ and $\operatorname{Per}\left(g^{\prime}\right)$. Moreover, we prove that every canonical model is, in particular, $y$-expansive. This allows us to use the results of Sec. 4 for canonical models. In Sec. 7 we prove Theorem A for a monotone model $(S, P, g)$. The complexity of the arguments of the proof depends strongly on the combinatorics of the $g$-invariant set $P \cup V(S)$ around a fixed point $y$ of $g$. This combinatorics is studied in Sec. 6, where we define the notion of a twist model around a fixed point and we remark that if $(S, P, g)$ is not a twist model around $y$ then the theorems of Sec. 4 can be directly used. The sets of periods of the twist models are studied in Sec. 6. In Sec. 8 we prove the inequalities (1). Finally Sec. 9 is devoted to prove Theorem B.

\section{Markov Graphs and Periodic Orbits}

Let $T$ be a tree and let $Q \subset T$ be a finite set containing $V(T)$. An interval of $T$ will be called $Q$-basic if it is the closure of a connected component of $T \backslash Q$. Given $f: T \longrightarrow T$ and $K, L \subset T$, we will say that $K f$-covers $L$ if $f(K) \supset L$. We will use the notation $K \rightarrow L$ (or $K \stackrel{f}{\rightarrow} L$ if we want to specify the map) to denote that $K f$-covers $L$. In this setting, it makes sense to consider the (Markov) $f$-graph of $Q$, whose vertices are $Q$-basic intervals and, if $I, J$ are $Q$-basic intervals, there is an arrow $I \rightarrow J$ if 
and only if $I f$-covers $J$.

The results of this section are well known for interval and star maps and extend straightforwardly to the case of tree maps. However, we include some proofs for completeness.

Lemma 3.1. Let $f: T \longrightarrow T$ be a tree map. Assume that $f$ is $Q$-monotone for a set $Q$ containing $V(T)$. Let $K \subset T$ be a connected union of $Q$-basic intervals. Then for each $Q$-basic interval $J \subset f(K)$ there exists a $Q$-basic interval $I \subset K$ such that $I$ $f$-covers $J$.

Proof. Note that $\operatorname{Int}(J) \cap V(T)=\emptyset$ because $J$ is a $Q$-basic interval and $V(T) \subset Q$. Since $f$ is continuous and $T$ is a tree, it follows that there exists an interval $I^{\prime} \subset K$ such that $f\left(I^{\prime}\right)=J$. Furthermore, since $f$ is $Q$-monotone we can assume $\operatorname{Int}\left(I^{\prime}\right) \cap V(T)=\emptyset$. Thus the lemma follows by taking a $Q$-basic interval $I$ such that $I^{\prime} \subset I \subset K$.

Let $f: T \longrightarrow T$ be a $Q$-monotone tree map, where $Q$ is a set which contains $V(T)$. There is a certain correspondence between periodic points of $f$ and loops in the $f$-graph of $Q$. We will use the usual notions (see Chapter 1 of [Alsedà et al., 2000] or [Block et al., 1980]): the concatenation of two loops $\alpha$ and $\beta$ will be denoted by $\alpha \beta$, and $\alpha^{n}=$ $\alpha \alpha \ldots \alpha$ ( $n$ times) will be called an $n$-repetition of $\alpha$. A loop will be called elementary if it cannot be formed by concatenating two loops. A loop $\alpha$ is simple if it is not an $n$-repetition of any other loop with $n \geq 2$. The length of a loop $\alpha$ will be denoted by $|\alpha|$. If $J_{0} \rightarrow J_{1} \rightarrow \ldots \rightarrow J_{n-1} \rightarrow J_{0}$ is a loop $\alpha$ in the $f$-graph of $Q$ and $x \in \operatorname{Fix}\left(f^{n}\right)$ we say that $x$ and $\alpha$ are associated if $f^{i}(x) \in J_{i}$ for $0 \leq i<n$. In this case we also will say that $\operatorname{Orb}(x)$ and $\alpha$ are associated. We note that when $x$ and $\alpha$ are associated the period of $x$ can be a strict divisor of $|\alpha|$. As usual, to every arrow $I \rightarrow$ $J$ in the $f$-graph of $Q$ we associate a sign which is +1 if $\left.f\right|_{I}$ is non-decreasing and -1 if it is nonincreasing. Then we say that the loop $J_{0} \rightarrow J_{1} \rightarrow$ $\ldots \rightarrow J_{n-1} \rightarrow J_{0}$ is positive if the product of the signs of the arrows $J_{0} \rightarrow J_{1}, J_{1} \rightarrow J_{2}, \ldots, J_{n-1} \rightarrow$ $J_{0}$ is +1 and negative if it is -1 .

Lemma 3.2. Let $f: T \longrightarrow T$ be a tree map. Assume that $f$ is $Q$-monotone for a set $Q$ containing $V(T)$. If $P$ is a periodic orbit of $f$ such that
$P \cap Q=\emptyset$, then there exists a loop $\alpha$ in the $f$-graph of $Q$ such that $P$ and $\alpha$ are associated.

Proof. Let $x \in P$. For each $0 \leq i<|P|$, there exists a unique $Q$-basic interval $J_{i}$ such that $f^{i}(x) \in$ $\operatorname{Int}\left(J_{i}\right)$. Since $f$ is $Q$-monotone and $V(T) \subset Q$, it follows that $J_{i} f$-covers $J_{i+1}$ for $0 \leq i<|P|-1$ and $J_{|P|-1} f$-covers $J_{0}$.

The next result follows easily from the ideas of Lemma 1.4 of [Block et al., 1980]. See also Lemma 2.1 of [Alsedà et al., 2001].

Lemma 3.3. Let $f: T \longrightarrow T$ be a tree map. Assume that $f$ is $Q$-monotone for a set $Q$ containing $V(T)$. Let $\alpha$ be a loop $J_{0} \rightarrow J_{1} \rightarrow \ldots \rightarrow J_{n-1} \rightarrow J_{0}$ in the $f$-graph of $Q$. Then there exist closed intervals $K_{i} \subset J_{i}$ for $0 \leq i<n$ such that $f\left(K_{i}\right)=K_{i+1}$ for $0 \leq i<n-1$ and $f\left(K_{n-1}\right)=J_{0}$. Moreover, there exists $x \in \operatorname{Fix}\left(f^{n}\right)$ such that $f^{i}(x) \in K_{i}$ for $0 \leq i<n$. In particular, $x$ and $\alpha$ are associated.

Remark 3.4. With the notation of Lemma 3.3, it is not difficult to see that $f^{n}$ is monotone on $K_{0}$, and the loop is positive (respectively negative) if and only if $\left.f^{n}\right|_{K_{0}}$ is non-decreasing (respectively nonincreasing).

Under the hypotheses of Lemma 3.3, there exists a periodic point $x$ associated to $\alpha$. Therefore, the loops of the $f$-graph of $Q$ are useful to obtain periodic orbits of the map $f$. When doing this, the basic problem is to determine the exact period of the periodic point that one gets. The following result imposes some conditions on $\alpha$ in order to assure that the period of $x$ coincides with the length of $\alpha$.

Lemma 3.5. Let $f: T \longrightarrow T$ be a tree map. Assume that $f$ is $Q$-monotone for a set $Q$ containing $V(T)$. Let $\alpha$ be a simple loop $[a, b] \rightarrow J_{1} \rightarrow J_{2} \rightarrow$ $\ldots \rightarrow J_{n-1} \rightarrow[a, b]$ in the $f$-graph of $Q$. Let $x$ be the periodic point given by Lemma 3.3. If $x \in(a, b)$ then the period of $x$ is $n$. This happens, in particular, when any of the following statements holds:

(a) $a$ and $b$ are not fixed points of $f^{n}$.

(b) $\alpha$ is negative.

Proof. We use the notation of Lemma 3.3. A standard argument (see for instance the first part of the 
proof of Lemma 1.2.11 of [Alsedà et al., 2000]) assures that, since $x \in \operatorname{Int}\left(J_{0}\right)$ and $\alpha$ is simple, the period of $x$ coincides with $|\alpha|=n$. Now we will see that $x \in \operatorname{Int}\left(J_{0}\right)$ when either (a) or (b) is satisfied.

When (a) holds, obviously $x \notin\{a, b\}$ since $x$ is a fixed point of $f^{n}$.

Assume that (b) holds. Set $K_{0}=[y, z] \subset J_{0}$. Then $x \in[y, z]$ and $f^{n}([y, z])=J_{0}$. Since $\alpha$ is negative, by Remark $3.4 f^{n}$ is monotone and nonincreasing on $[y, z]$. Since $x \in \operatorname{Fix}\left(f^{n}\right)$, it follows that $x \neq y$ and $x \neq z$. Thus $x \in(y, z) \subset \operatorname{Int}\left(J_{0}\right)$.

In view of Lemma 3.5, simple loops are specially useful to calculate periodic orbits. The following lemma gives a tool to obtain a simple loop from a given one.

Lemma 3.6. For each loop $\gamma$ which is not a repetition of an elementary loop there exists a simple loop which can be obtained by permuting the elements of $\gamma$.

Proof. Clearly $\gamma$ can be written as $\alpha^{l} \beta$ for some $l \in \mathbb{N}$, a non-empty elementary loop $\alpha$ and $|\beta|>$ 0 . If $\beta=\beta^{\prime} \alpha \beta^{\prime \prime}$ (where either $\beta^{\prime}$ or $\beta^{\prime \prime}$ can be empty) then the loop $\gamma^{\prime}=\alpha^{l+1} \beta^{\prime} \beta^{\prime \prime}$ is obtained by permuting the elements of $\gamma$. By iterating this procedure, if necessary, we obtain a loop $\tilde{\gamma}=\alpha^{r} \tilde{\beta}$ which is a permutation of the elements of $\gamma$ such that $r \in \mathbb{N},|\tilde{\beta}|>0$ and $\tilde{\beta}$ does not contain $\alpha$. Clearly $\tilde{\gamma}$ is simple.

\section{Periodic Orbits in $y$-expansive Monotone Models}

In this section we introduce a particular class of monotone models, which will be called $y$-expansive. This kind of models satisfy certain properties of expansivity around a fixed point $y$. We study the Markov graph of $y$-expansive models and derive the structure of the set of periods.

Let $T$ be a tree. Given a point $y \in T$, a (partial) ordering among the points of $T$ may be defined: for $z, z^{\prime} \in T$, we write $z \prec_{y} z^{\prime}$ if and only if $z \in\left[y, z^{\prime}\right)$. We remark that if $\left(z, z^{\prime}\right) \cap(V(T) \cup\{y\})=\emptyset$ then either $z \prec_{y} z^{\prime}$ or $z^{\prime} \prec_{y} z$. The notations ${ }_{y} \succ, \preceq_{y}$ and $y \succeq$ will be understood in the natural way, and for simplicity we will omit the subindex $y$ when no confusion seems possible. If $I, J$ are subsets of $T$, we will write $I \prec_{y} J$ if $z \prec_{y} z^{\prime}$ for each $z \in \operatorname{Int}(I)$ and $z^{\prime} \in \operatorname{Int}(J)$.

Given a finite set $Q \subset T$ and a point $y \in T$, we shall denote by $Z^{\star}(Q)$ the connected component of $(T \backslash Q) \cup\{y\}$ which contains $y$. Let $n$ be the number of connected components of $T \backslash Z^{\star}(Q)$. These connected components will be denoted by $Z(Q)_{i}$ for $1 \leq i \leq n$ and we will call them $y$ branches. The set $\mathrm{Cl}\left(Z^{\star}(Q)\right) \cap Z(Q)_{i}$ consists of a single point which belongs to $Q$. This point will be denoted by $x(Q)_{i}$. We remark that, for each $z \in T, z \in Z(Q)_{i}$ if and only if $x(Q)_{i} \preceq_{y} z$. Finally we set $X(Q)=\left\{x(Q)_{i}\right\}_{i=1}^{n}$.

Let $f: T \longrightarrow T$ be a tree map, $y \in \operatorname{Fix}(f)$ and let $Q \subset T$ be a finite $f$-invariant set. We will say that $Q$ is $y$-typifiable if $f(X(Q)) \cap Z^{\star}(Q)=\emptyset$.

Remark 4.1. If $y \notin Q$ then $Q$ is $y$-typifiable. If $y \in$ $Q$ then $Q$ is $y$-typifiable if and only if $f\left(x(Q)_{i}\right) \neq y$ for $1 \leq i \leq|X(Q)|$. Moreover, it follows that $Q$ is $y$-typifiable if and only if $Q \cup\{y\}$ is $y$-typifiable.

If $Q$ is $y$-typifiable then we consider the map

$$
\Phi_{Q}: X(Q) \longrightarrow X(Q)
$$

defined by $\Phi_{Q}\left(x(Q)_{i}\right)=x(Q)_{j}$ if and only if $f\left(x(Q)_{i}\right) \in Z(Q)_{j}$. Observe that $\Phi_{Q}$ is well defined and, since it acts on a finite set, it has periodic orbits. The period $p$ of a periodic orbit of $\Phi_{Q}$ will be called a type of $Q$ (note that the type of a $y$-typifiable set is not necessarily unique).

Given a type $p$ of $Q$, in what follows we will assume that the $y$-branches are indexed in such a way that $f\left(x(Q)_{i}\right) \in Z(Q)_{i+1} \bmod p$ for $1 \leq i \leq p$.

Observe that all the definitions introduced up to now in this section depend on the chosen point $y$. For simplicity, this dependence is not made explicit in the notation.

Lemma 4.2. Let $f: T \longrightarrow T$ be a tree map. Let $y \in \operatorname{Fix}(f)$ and let $Q \subset T$ be a $y$-typifiable set. If $p$ is a type of $Q$ then $p \in \operatorname{Per}(f)$.

Proof. Let $r: T \longrightarrow \mathrm{Cl}\left(Z^{\star}\right)$ be the natural retraction. Clearly $r\left(f\left(x(Q)_{i}\right)\right)=x(Q)_{i+1} \bmod p$ for $i=1,2, \ldots, p$. Then $x(Q)_{1}$ is a $p$-periodic point of $r \circ f$ and thus $p \in \operatorname{Per}(r \circ f)$. The lemma follows because $\operatorname{Per}(r \circ f) \subset \operatorname{Per}(f)$ (see, for instance, Corollary 4.2 of [Baldwin, 1991]). 


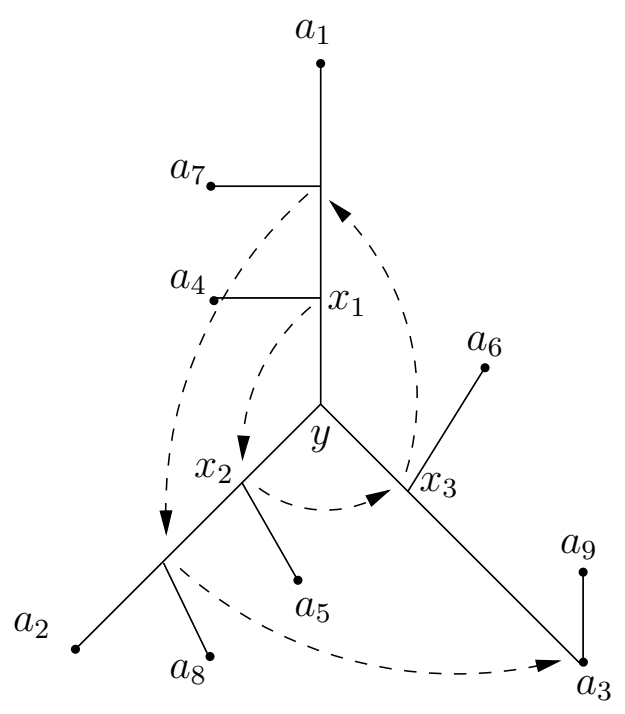

Fig. 2. A $y$-expansive model $(T, A, f)$ with $A=\left\{a_{i}\right\}_{i=1}^{9}$ and $f\left(a_{i}\right)=a_{i+1}$ mod 9 . For this model, $\mathrm{Cl}\left(Z^{\star}\right)=$ $\left\langle\left\{x_{1}, x_{2}, x_{3}\right\}\right\rangle, A^{y}$ has a unique type $p=3$ and $q_{1}=5, q_{2}=4, q_{3}=3$. Therefore, the rotation index associated to the type is 3 .

Let $(T, A, f)$ be a monotone model. It is not difficult to prove that if $B \subset T$ is finite and $f$ invariant then $f$ is $(A \cup B)$-monotone. Thus, since $A \cup V(T)$ is an $f$-invariant set by Remark 2.1, $f$ is also $(A \cup V(T))$-monotone. We will say that a monotone model $(T, A, f)$ is $y$-expansive for $y \in$ $\operatorname{Fix}(f) \backslash A$ if $\operatorname{Orb}_{f}(v)$ is not contained in $Z^{\star}(A)$ for every $v \in V(T) \backslash\{y\}$. This sort of models will play an important role in this paper. Lemma 4.3 states that on $y$-expansive models it is possible to define the type of some "natural" invariant sets.

Lemma 4.3. Let $(T, A, f)$ be a y-expansive model. Let $P \subset T$ be a finite (or empty) $f$-invariant set such that $y \notin P$. Then the sets $A, A \cup\{y\}, P$, $P \cup\{y\}, A \cup V(T) \cup P$ and $A \cup V(T) \cup P \cup\{y\}$ are $y$-typifiable.

Proof. Let $Q=A \cup V(T) \cup P$. By Remark 4.1, to prove the lemma it suffices to show that the sets $A, P$ and $Q$ are $y$-typifiable. Since $y \notin A$ and $y \notin P$, it follows that $A$ and $P$ are $y$-typifiable. If in addition $y \notin V(T)$ then $y \notin Q$ and we are done. Assume that $y \in V(T)$. Then $y \in Q$ and we must prove that if $i \in\{1,2, \ldots,|X(Q)|\}$ then $f\left(x(Q)_{i}\right) \neq y$. This is obvious if $x(Q)_{i} \in$ $A \cup P$. Assume that $x(Q)_{i} \in V(T) \backslash(A \cup P)$. We note that $\left(y, x(Q)_{i}\right) \cap Q=\emptyset$ and, in particular, $\left(y, x(Q)_{i}\right) \cap A=\emptyset$. Then, as an immediate consequence of the fact that $\operatorname{En}(T) \subset A$, we have that $x(Q)_{i} \prec x(A)_{j}$ for some $j \in\{1,2, \ldots,|X(A)|\}$. This is equivalent to $x(Q)_{i} \in Z^{\star}(A)$, and then $f\left(x(Q)_{i}\right) \neq y$ since $(T, A, f)$ is $y$-expansive.

Let $(T, A, f)$ be a $y$-expansive model. The set $A \cup V(T) \cup\{y\}$ will be denoted by $A^{y}$, and $\left|X\left(A^{y}\right)\right|$ will be denoted by $n^{\star}$. Furthermore, from now on we will write $Z^{\star}, Z_{i}$ and $x_{i}$ instead of $Z^{\star}\left(A^{y}\right)$, $Z\left(A^{y}\right)_{i}$ and $x\left(A^{y}\right)_{i}$, for $1 \leq i \leq n^{\star}$.

By Lemma 4.3, $A^{y}$ is $y$-typifiable. Let $p$ be a type of $A^{y}$. For each $i \in\{1,2, \ldots, p\}$ there exists a non-negative number, which we will denote by $q_{i}$, such that $\left[y, f^{j}\left(x_{i}\right)\right] \cap A=\emptyset$ for $0 \leq j<q_{i}$ and $\left[y, f^{q_{i}}\left(x_{i}\right)\right] \cap A \neq \emptyset$ (recall that $Z^{\star}=Z^{\star}\left(A^{y}\right) \subset$ $Z^{\star}(A)$ ). Note that $x_{i} \in A$ if and only if $q_{i}=0$. The non-negative integer $\min \left\{q_{1}, q_{2}, \ldots, q_{p}\right\}$ will be called a rotation index of $(T, A, f)$ associated to the type $p$. Observe that the rotation index associated to a type $p$ of $A^{y}$ is not unique, since it depends on the chosen $p$-periodic orbit of $\Phi_{A^{y}}$.

The following technical lemma concerns the dynamical behavior of a $y$-expansive model near the fixed point $y$. See Fig. 2 for an example.

Lemma 4.4. Let $(T, A, f)$ be a y-expansive model and let $p$ be a type of $A^{y}$. Let $k \in\{1,2, \ldots, p\}$ be such that $q_{k}>0$. Then $x_{k+i} \bmod p \preceq_{y} f^{i}\left(x_{k}\right)$ for $1 \leq i \leq q_{k}$ and $f^{i-p}\left(x_{k}\right) \prec_{y} f^{i}\left(x_{k}\right)$ for $p<i \leq q_{k}$. 
Proof. In the whole proof, the subindexes will be considered modulo $p$.

We will prove the first statement by induction on $i$. From the definition of type, it follows that $x_{k+1} \preceq_{y} f\left(x_{k}\right)$. Hence, the first statement holds for $i=1$. Now take $1<i \leq q_{k}$ and assume that $y \prec_{y} x_{k+i-1} \preceq_{y} f^{i-1}\left(x_{k}\right)$. Since $i-1<q_{k}$, the definition of $q_{k}$ implies that $\left[y, f^{i-1}\left(x_{k}\right)\right] \cap A=\emptyset$. Thus, from the $A$-monotonicity of $f$ it follows that $f\left(x_{k+i-1}\right) \preceq_{y} f\left(f^{i-1}\left(x_{k}\right)\right)=f^{i}\left(x_{k}\right)$. Since from the definition of type $x_{k+i} \preceq_{y} f\left(x_{k+i-1}\right)$, the first statement is proved.

Let us prove the second statement also by induction on $i$. Since $q_{k}>0$, we have that $x_{k} \in V(T)$. We assume that $p<q_{k}$ since otherwise there is nothing to prove. For $i=p+1$ we must show that $f\left(x_{k}\right) \prec_{y} f^{p+1}\left(x_{k}\right)$. Since $p<q_{k}$, we know from the first statement that $y \prec_{y} x_{k} \preceq_{y} f^{p}\left(x_{k}\right)$. The fact that $f$ is $A$-motonone implies, as above, that $f\left(x_{k}\right) \preceq_{y} f^{p+1}\left(x_{k}\right)$. If $f\left(x_{k}\right)=f^{p+1}\left(x_{k}\right)$, since $p<q_{k}$ it follows that $\operatorname{Orb}\left(x_{k}\right)$ is a finite $f$-invariant set contained in $\left(Z^{\star}(A) \cap V(T)\right) \backslash\{y\}$. This contradicts the fact that $(T, A, f)$ is $y$-expansive and proves that $f\left(x_{k}\right) \prec_{y} f^{p+1}\left(x_{k}\right)$.

Now take $p+1<i \leq q_{k}$ and assume that $f^{i-1-p}\left(x_{k}\right) \prec_{y} f^{i-1}\left(x_{k}\right)$. Then we obtain that $f^{i-p}\left(x_{k}\right) \prec_{y} f^{i}\left(x_{k}\right)$ in the same way as above.

Let $(T, A, f)$ be a $y$-expansive model. Let $p$ be a type of $A^{y}$. For $i \in\{1,2, \ldots, p\}$, we write $I_{i}$ for $\left[y, x_{i}\right]$. We note that these sets are $A^{y}$-basic intervals, and they are contained in $\mathrm{Cl}\left(Z^{\star}\right)$. Moreover, by the definition of $p$, the $f$-graph of $A^{y}$ contains the loops $I_{i \bmod p} \rightarrow I_{i+1} \bmod p \rightarrow \ldots \rightarrow I_{i+p} \bmod p$, which will be called typical loops. The intervals $I_{1}, I_{2}, \ldots, I_{p}$ will be called typical intervals.

Remark 4.5. Assume that a typical interval $I_{i} f$ covers an interval $J$ which is not typical. Since $f(y)=y$ and $\left.f\right|_{I_{i}}$ is monotone, it follows that $I_{i+1} \bmod p \prec_{y} J$.

The periods of $f$ obtained in this section (see Lemma 4.6 and Theorem 4.7) will be computed by linking the typical loops with some special loops of the Markov $f$-graph of $A^{y}$. A loop in the $f$-graph of $A^{y}$ will be called external if it starts and ends at a typical interval and it contains an element which is not a typical interval. We denote by $\mathcal{E}(T, A, f)$ the set of external loops in the $f$-graph of $A^{y}$. Observe that the notions of typical interval and external loop depend on the point $y$, the type $p$ and the chosen $p$ periodic orbit of $\Phi_{A^{y}}$. For simplicity, the notations do not take it into account.

Next we state and prove two results that allow us to obtain periodic orbits in the context of $y$ expansive models.

Lemma 4.6. Let $(T, A, f)$ be a y-expansive model and let $p$ be a type of $A^{y}$. If $\beta \in \mathcal{E}(T, A, f)$ then $\{|\beta| i+p j: i, j \geq 1\} \subset \operatorname{Per}(f)$.

Proof. Since $\beta$ is external, $\beta$ starts and ends at a typical interval $I_{t}$. Let $\alpha$ be the typical loop starting and ending at $I_{t}$. Set $k=|\beta| i+p j$ with $i, j \geq 1$. We consider the loop $\alpha^{j} \beta^{i}$, whose length is $k$. Since $\beta$ is external, $\alpha^{j} \beta^{i}$ is not a repetition of $\alpha$. So, Lemma 3.6 gives us a simple loop $\gamma$ obtained by permuting the elements of $\alpha^{j} \beta^{i}$. By Lemma 3.3, there is a point $x \in T$ associated to $\gamma$ such that $f^{k}(x)=x$. Since $j \geq 1$, we can assume that $x \in I_{t}$ and $f^{n}(x) \in I_{t+n} \bmod p$ for $1 \leq n \leq p$.

By Lemma 3.5, it is enough to prove that $x \in \operatorname{Int}\left(I_{t}\right)$. First we show that $x \neq y$. Since $\beta$ is external, $\gamma$ contains an arrow $I_{r} \rightarrow J$ for some $r \in\{1,2, \ldots, p\}$ and some $J$ which is not a typical interval. Then by Remark $4.5 I_{r+1} \bmod p \prec_{y} J$ and thus $y \notin J$. Since some iterate of $x$ belongs to $J$, it follows that $x \neq y$. To end the proof of the claim we must show that $x \neq x_{t}$. Suppose that $x=x_{t}$. Then clearly $f^{n}(x)=x_{t+n} \bmod p$ for $1 \leq n \leq p$ and thus $f^{p}(x)=x$. Since $f$ is monotone on each typical interval, it follows that for each $1 \leq n \leq p$, $I_{n+1} \bmod p$ is the only $A^{y}$-basic interval $f$-covered by $I_{n}$. This contradicts the existence of the arrow $I_{r} \rightarrow J$.

Let $(T, A, f)$ be a monotone model. We say that $(T, A, f)$ is orbital if $A$ contains a unique periodic orbit which is not a fixed point and there is at most one endpoint of $T$ that does not belong to this periodic orbit. Observe that there exists $n \geq 0$ such that, for each $x \in A, f^{n}(x)$ belongs to the periodic orbit. Then we will also say that $(T, A, f)$ is $n$-orbital. We note that an $n$-orbital model is also $(n+k)$-orbital for all $k \geq 0$. Obviously if $A$ is a periodic orbit then $(T, A, f)$ is 0 -orbital.

Given a map $f$ and an $f$-invariant set $A$ containing a unique periodic orbit, we will denote this periodic orbit by $A^{\circ}$. 
Theorem 4.7. Let $(T, A, f)$ be a y-expansive $n$ orbital model. Let $p$ be a type of $A^{y}$ and let $q$ be a rotation index associated to the type $p$. If $\left|A^{\circ}\right| \notin p \mathbb{N}$ then $\mathcal{E}(T, A, f) \neq \emptyset$ and $\operatorname{Per}(f) \supset\left\{\left(\left|A^{\circ}\right|+l p\right) i+p j\right.$ : $i, j \geq 1\}$ for some $0 \leq l \leq\left|A^{\circ}\right|+q+n-1$. Furthermore, if $n=0$ then $l p \leq p+q-(q \bmod p)$.

Proof. In the whole proof, the subindexes will be considered modulo $p$. Let $\alpha$ be the typical loop starting at $I_{p}$. We can assume without loss of generality (by reindexing, if necessary) that $q=q_{p}$. Note that the assumption $\left|A^{\circ}\right| \notin p \mathbb{N}$ implies, in particular, that $p>1$.

Since $(T, A, f)$ is $y$-expansive and $n$-orbital, there exists $z \in f^{r}\left(\left[y, x_{p}\right]\right) \cap A^{\circ}$ for some $r \leq q+n$. Furthermore, $\left|\operatorname{En}(T) \backslash A^{\circ}\right| \leq 1$ and thus each $y$-branch $Z_{1}, Z_{2}, \ldots, Z_{p}$ (except, at most, one of them) contains at least one endpoint of $T$ which belongs to $A^{\circ}$. Since $z \in A^{\circ}$ and $A^{\circ}$ is a periodic orbit, it follows easily that there exists $s \leq\left|A^{\circ}\right|-p$ such that $f^{s}(z) \succeq x_{j}$ for some $j \in\{1,2, \ldots, p\}$.

Since $y \prec x_{j} \preceq f^{s}(z)$, we have that $I_{j}=$ $\left[y, x_{j}\right] \subset\left[y, f^{s}(z)\right]$ and therefore $f\left(\left[y, f^{s}(z)\right]\right) \supset$ $f\left(I_{j}\right) \supset I_{j+1}$. In other words, $\left[y, f^{s}(z)\right] f$-covers $I_{j+1}$. Furthermore, $I_{p} f^{r}$-covers $[y, z],[y, z] f^{s}$ covers $\left[y, f^{s}(z)\right]$ and $\left[y, f^{s}(z)\right] f^{\left|A^{\circ}\right|}$-covers itself. Therefore we have the following sequence of coverings:

$$
\begin{gathered}
I_{p} \stackrel{f^{r}}{\longrightarrow}[y, z] \stackrel{f^{s}}{\longrightarrow}\left[y, f^{s}(z)\right] \stackrel{f^{\left|A^{\circ}\right|}}{\longrightarrow}\left[y, f^{s}(z)\right] \rightarrow \\
\rightarrow I_{j+1} \rightarrow I_{j+2} \rightarrow \ldots \rightarrow I_{p} .
\end{gathered}
$$

Then, by using Lemma 3.1 by backwards induction, we obtain a loop $\gamma$ in the $f$-graph of $A^{y}$ such that $|\gamma|=r+s+\left|A^{\circ}\right|+p-j$. On the other hand, we can also consider the following sequence of coverings:

$$
\begin{aligned}
& I_{p} \stackrel{f^{r}}{\longrightarrow}[y, z] \stackrel{f^{s}}{\longrightarrow}\left[y, f^{s}(z)\right] \rightarrow \\
& \rightarrow I_{j+1} \rightarrow I_{j+2} \rightarrow \ldots \rightarrow I_{p} .
\end{aligned}
$$

Again by using Lemma 3.1 by backwards induction we obtain a loop $\sigma$ in the $f$-graph of $A^{y}$ such that $|\sigma|=r+s+p-j$. Let $\beta$ be the loop $\gamma \sigma^{p-1}$, whose length is $\left|A^{\circ}\right|+l p$ with $l=r+s+p-j$. Note that $l \leq q+n+\left|A^{\circ}\right|-p+p-j \leq q+n+$ $\left|A^{\circ}\right|-1$. We claim that $\beta$ is external. Indeed, if all the intervals of $\beta$ were typical, by Remark 4.5, $\beta$ would be a repetition of $\alpha$ and then $|\beta| \in p \mathbb{N}$, in contradiction with the fact that $\left|A^{\circ}\right| \notin p \mathbb{N}$. This proves the claim. By Lemma 4.6 we obtain that $\operatorname{Per}(f) \supset\left\{\left(\left|A^{\circ}\right|+l p\right) i+p j: i, j \geq 1\right\}$.

Finally note that, when $n=0, A=A^{\circ}$. Moreover, it is not difficult to see that Lemma 4.4 gives $r=q, s=0, z=x_{r}$ and $j=r \bmod p$ in the above construction of the loop $\beta$. Hence $|\beta|=\left|A^{\circ}\right|+l p=\left|A^{\circ}\right|+q+p-(q \bmod p)$.

\section{Canonical and Monotone Models}

In this section we use the notion of a canonical model introduced in [Alsedà et al., 1997]. From a monotone model $(S, B, g)$, a canonical model $(T, A, f)$ can be constructed, essentially, by collapsing the $V(S)$-basic intervals whose orbit does not intersect $B$. We prove that $\operatorname{Per}(f) \subset \operatorname{Per}(g)$ and that $\operatorname{Per}(g) \backslash \operatorname{Per}(f)$ is finite.

We start by recalling the definition of a canonical model. Let $(S, B, g)$ be a monotone model. We will say that $v_{1}, v_{2} \in V(S) \backslash B$ are $g$-identifiable if either:

(i) $\left[g^{i}\left(v_{1}\right), g^{i}\left(v_{2}\right)\right] \cap B=\emptyset$ for all $i \geq 0$, or

(ii) if $\left[g^{n}\left(v_{1}\right), g^{n}\left(v_{2}\right)\right] \cap B \neq \emptyset$ for some $n \geq 0$ then $g^{n}\left(v_{1}\right)=g^{n}\left(v_{2}\right)$.

Since $g$ is $B$-monotone, it is easy to check that the $g$-identifiability is an equivalence relation. Moreover, since $V(S)$ is finite, there are finitely many equivalence classes.

Remark 5.1. From Remark 2.1 it follows that:

(i) If $v_{1}, v_{2}$ are $g$-identifiable then $g^{i}\left(v_{1}\right), g^{i}\left(v_{2}\right)$ are $g$-identifiable for each $i \geq 0$ such that $g^{i}\left(v_{1}\right), g^{i}\left(v_{2}\right) \in V(S) \backslash B$.

(ii) If $v_{1}$ and $v_{2}$ are $g$-identifiable and $v_{3} \in$ $\left[v_{1}, v_{2}\right] \cap V(S)$ then $v_{1}, v_{2}, v_{3}$ are pairwise $g$ identifiable.

A monotone model $(T, A, f)$ such that every class of the $f$-identifiability relation contains exactly one point will be called a canonical model.

The following technical lemma is used in the proof of Theorem 5.3.

Lemma 5.2. Let $(S, B, g)$ be a monotone model and let $\left[v, v^{\prime}\right]$ be a $V(S)$-basic interval such that $v, v^{\prime} \in V(S) \backslash B$ are g-identifiable. Let $x \in\left(v, v^{\prime}\right)$. Then either $x$ is not periodic or there exist $k, n, n^{\prime}$ such that $g^{k}(v)$ is $n$-periodic, $g^{k}\left(v^{\prime}\right)$ is $n^{\prime}$-periodic 
and $x \in \operatorname{Fix}\left(g^{m}\right)$, where $m$ is the least common multiple of $n$ and $n^{\prime}$.

Proof. Since $v$ and $v^{\prime}$ are $g$-identifiable, $\left[v, v^{\prime}\right] \cap$ $B=\emptyset$. Furthermore, the $B$-monotonicity of $g$ implies that $g^{i}$ is monotone on $\left[v, v^{\prime}\right]$ for every $i \geq 0$ such that $\left[g^{i}(v), g^{i}\left(v^{\prime}\right)\right] \cap B=\emptyset$. In particular, $g^{i}\left(\left[v, v^{\prime}\right]\right)=\left[g^{i}(v), g^{i}\left(v^{\prime}\right)\right]$ and thus $g^{i}(x) \in$ $\left[g^{i}(v), g^{i}\left(v^{\prime}\right)\right]$. Hence, if there exists $n \geq 1$ such that $g^{n}(v)=g^{n}\left(v^{\prime}\right) \in B$ then $g^{n}\left(\left[v, v^{\prime}\right]\right)$ reduces to a point of $B$. Therefore, there are no periodic points in $\left(v, v^{\prime}\right)$ and we are done.

Assume now that $\left[g^{i}(v), g^{i}\left(v^{\prime}\right)\right] \cap B=\emptyset$ for all $i \geq 0$. Since $V(S)$ is finite, there exist $r, r^{\prime} \geq 0$ such that $g^{r}(v)$ and $g^{r^{\prime}}\left(v^{\prime}\right)$ are periodic points. Take $k=\max \left\{r, r^{\prime}\right\}$. Then $g^{k}(v)$ and $g^{k}\left(v^{\prime}\right)$ are periodic points. Let $n$ and $n^{\prime}$ be their respective periods, and let $m$ be the least common multiple of $n$ and $n^{\prime}$. Then $g^{k}(v)$ and $g^{k}\left(v^{\prime}\right)$ are fixed points of $g^{m}$. Since $g^{m}$ is monotone on $\left[g^{k}(v), g^{k}\left(v^{\prime}\right)\right]$, it follows that $\operatorname{Per}\left(\left.g^{m}\right|_{\left[f^{k}(v), f^{k}\left(v^{\prime}\right)\right]}\right)=\{1\}$. Therefore, either $g^{k}(x)$ is not periodic or $g^{k}(x)$ is a fixed point of $g^{m}$. Observe that if $x$ is periodic then the periods of $x$ and $g^{k}(x)$ are the same. Thus either $x$ is not periodic or it is a fixed point of $g^{m}$. This ends the proof.

Theorem 5.3. Let $(S, B, g)$ be a monotone model. There exists a canonical model $(T, A, f)$ and a (possibly empty) finite set $\mathcal{V}$ such that

$$
\operatorname{Per}(g)=\operatorname{Per}(f) \cup \mathcal{V}
$$

and each element of $\mathcal{V}$ divides the least common multiple of the periods of all periodic orbits of $g$ contained in $V(S)$. Moreover, $|A|=|B|$ and if $(S, B, g)$ is $k$-orbital then $(T, A, f)$ is $k$-orbital.

Proof. Let $K$ be the union of the convex hulls of all the classes of the $g$-identifiability relation. We remark that $K$ has finitely many connected components, each of them contained in a connected component of $S \backslash B$. Let $T$ be the tree obtained by contracting each connected component of $K$ to a point and let $\phi: S \longrightarrow T$ be the standard projection. That is, $\phi$ is injective in a neighborhood of each point which does not belong to $K$, and the image of each point in a connected component $C$ of $K$ is the point to which $C$ is contracted.

We define $f: T \longrightarrow T$ by $f(x)=\phi\left(g\left(x^{\prime}\right)\right)$, where $x^{\prime} \in \phi^{-1}(x)$. By Remark 5.1, $f$ is well defined. Set $A=\phi(B)$. Then $|A|=|B|$ and the fact that $g$ is $B$-monotone implies that $f$ is $A$ monotone. Furthermore, if $v, v^{\prime} \in V(T) \backslash A$ are $f$ identifiable then $v=v^{\prime}$. Hence $(T, A, f)$ is a canonical model. Moreover, since $\operatorname{En}(T)=\phi(\operatorname{En}(S))$ and $f \circ \phi=\phi \circ g$, we easily get that if $(S, B, g)$ is $k$-orbital then $(T, A, f)$ is $k$-orbital.

To end the proof of the theorem, it remains to show that $\operatorname{Per}(g)=\operatorname{Per}(f) \cup \mathcal{V}$ for a finite set $\mathcal{V}$ verifying the prescribed properties. To do it, we claim that $B \cup K$ is $g$-invariant. Let us prove the claim. Since $B$ is $g$-invariant, it is enough to show that the orbit of each point of $K$ lies in $B \cup K$. Let $x \in K$. Assume first that $x \in V(S)$. Then $f^{i}(x) \in V(S) \cup B$ for all $i \geq 0$. Since each vertex of $S$ belongs either to $B$ or to its own $g$-identifiability class, we have that $V(S) \subset B \cup K$. Thus the claim follows in this case. Assume now that $x \notin V(S)$. Then there exist $v, v^{\prime} \in V(S) \backslash B$ such that $v$ and $v^{\prime}$ are $f$-identifiable and $x \in\left(v, v^{\prime}\right)$. By Remark 5.1, $g^{i}(v)$ and $g^{i}\left(v^{\prime}\right)$ are $f$-identifiable for each $i \geq 0$ such that $\left[g^{i}(v), g^{i}\left(v^{\prime}\right)\right] \cap B=\emptyset$. So, $\left[g^{i}(v), g^{i}\left(v^{\prime}\right)\right] \subset$ $K$. Furthermore, since $g$ is $B$-monotone, $g^{i}(x) \in$ $\left[g^{i}(v), g^{i}\left(v^{\prime}\right)\right]$. Then it is clear that $g^{i}(x) \in K \cup B$ for all $i \geq 0$. Thus the claim is proved.

Since $B \cup K$ is $g$-invariant and $f \circ \phi=\phi \circ g$, $\phi(B \cup K)$ is $f$-invariant. Clearly, if $x \in S \backslash(K \cup B)$ is a periodic point of $g$ then $\operatorname{Orb}_{g}(v) \subset S \backslash(B \cup$ $K)$. Furthermore, $\phi(x)$ is a periodic point of $f$ of the same period, and $\operatorname{Orb}_{f}(\phi(x)) \subset T \backslash \phi(K \cup B)$. Conversely, if $x \in T \backslash \phi(K \cup B)$ is a periodic point of $f$ then $\operatorname{Orb}_{f}(x) \subset T \backslash \phi(K \cup B), \phi^{-1}(x)$ is a periodic point of $g$ of the same period and $\operatorname{Orb}_{g}\left(\phi^{-1}(x)\right) \subset$ $S \backslash(K \cup B)$. Therefore, in order to complete the proof it is enough to show that

$$
\operatorname{Per}\left(\left.g\right|_{K \cup B}\right)=\operatorname{Per}\left(\left.f\right|_{\phi(K \cup B)}\right) \cup \mathcal{V}
$$

for some finite (or empty) set $\mathcal{V}$ satisfying the prescribed properties. From Lemma 5.2 and the fact that $V(S)$ is finite, we easily get that $\operatorname{Per}\left(\left.g\right|_{K \cup B}\right)$ is finite. Furthermore, for each $n$-periodic orbit of $\left.g\right|_{K \cup B}$ there exist two periodic orbits of $g$ contained in $V(S)$ in such a way that $n$ divides the least common multiple of their periods. Thus it suffices to show that $\operatorname{Per}\left(\left.f\right|_{\phi(K \cup B)}\right) \subset \operatorname{Per}\left(\left.g\right|_{K \cup B}\right)$.

Since $\left.f \circ \phi\right|_{B}=\left.g\right|_{B}$, it is enough to show that for each $n$-periodic point of $f$ in $\phi(K)$ there exists an $n$-periodic point of $g$ in $K$. Let $x \in \phi(K)$ be 
an $n$-periodic point of $f$. Let $K_{i}=\phi^{-1}\left(f^{i}(x)\right)$ for $i=1,2, \ldots, n$. By the definition of $\phi$, each $K_{i}$ is the convex hull of a class of $g$-identifiability and contains points of $V(S) \backslash B$. Furthermore, $K_{i} \neq K_{j}$ if $i \neq j$ since $f^{i}(x) \neq f^{j}(x)$. By the definition of $f$, for $i=1,2, \ldots, n$ we have that $f^{i+1}(x)=f\left(f^{i}(x)\right)=$ $\phi\left(g\left(x_{i}\right)\right)$ for some $x_{i} \in \phi^{-1}\left(f^{i}(x)\right)=K_{i}$. We choose $x_{i} \in V\left(K_{i}\right)$ for each $i$. Take $i \in\{1,2, \ldots, n\}$. Then we have that $\phi\left(g\left(x_{i}\right)\right)=f\left(\phi\left(x_{i}\right)\right)=f\left(f^{i}(x)\right)=$ $f^{i+1}(x)$. Therefore, $g\left(x_{i}\right) \in K_{i+1} \bmod n$. Moreover, $g\left(K_{i}\right) \subset K_{i+1} \bmod n$. Indeed, for each $z \in K_{i}$ there exists $v \in \operatorname{En}\left(K_{i}\right)$ such that $z \in\left[v, x_{i}\right]$ and $g$ is monotone on $\left[v, x_{i}\right]$. Since $g\left(x_{i}\right) \in K_{i+1} \bmod n$ and $g(v)$ and $g\left(x_{i}\right)$ are $g$-identifiable, $g(v) \in K_{i+1} \bmod n$ and thus $g(z) \in K_{i+1} \bmod n$.

From above we have $g^{i}\left(K_{1}\right) \subset K_{i+1} \bmod n$ for $i \geq 0$, and hence $g^{n}\left(K_{1}\right) \subset K_{1}$. Then there exists a fixed point of $g^{n}$ in $K_{1}$, which is obviously a point of period $n$ of $g$.

Let $(S, P, g)$ be a monotone model and let $(T, A, f)$ be the canonical model constructed from $(S, P, g)$ as in the proof of Theorem 5.3. We will say that $(S, P, g)$ and $(T, A, f)$ are associated to each other. With this notion, Theorem 5.3 can be restated as follows: each monotone model admits an associated canonical model. This theorem allows us to restrict our attention to the study of the set of periods of canonical models rather than to generic monotone models.

The following proposition says that a canonical model is $y$-expansive, and therefore all the results of Sec. 4 can be applied to canonical models. This fact will be used in the rest of the paper.

Proposition 5.4. If $(T, A, f)$ is an orbital canonical model, then there exists a fixed point $y$ of $f$ such that $(T, A, f)$ is y-expansive.

Proof. Since $(T, A, f)$ is orbital, $A$ does not contain fixed points and therefore $\operatorname{Fix}(f) \backslash A \neq \emptyset$. If $V(T) \cap \operatorname{Fix}(f) \neq \emptyset$, we take $y \in V(T) \cap \operatorname{Fix}(f)$. Otherwise we take any $y \in \operatorname{Fix}(f)$. Let $v \in(V(T) \cap$ $\left.Z^{\star}(A)\right) \backslash\{y\}$ (if $v$ does not exist then $(T, A, f)$ is obviously $y$-expansive). By Remark $2.1, \operatorname{Orb}(v) \subset$ $A \cup V(T)$. Assume that $\operatorname{Orb}(v) \subset Z^{\star}(A)$ (in particular, $\operatorname{Orb}(v) \cap A=\emptyset$ and hence $\operatorname{Orb}(v) \subset V(T))$ and we will arrive to a contradiction. If $v \in \operatorname{Fix}(f)$ then the choice of $y$ implies that $y \in V(T)$. Since $f$ is $A$ monotone and $[y, v] \cap A=\emptyset,[y, v]=\left[f^{i}(y), f^{i}(v)\right]$ for each $i \geq 0$. Thus $y$ and $v$ are $f$-identifiable, a contradiction with the fact that $(T, A, f)$ is a canonical model.

Assume now that there exist $z, z^{\prime} \in \operatorname{Orb}(v) \subset$ $V(T)$ such that $z \neq z^{\prime}$. Then, as above, the $A$ monotonicity of $f$ implies that $\left[f^{i}(z), f^{i}\left(z^{\prime}\right)\right] \cap A=\emptyset$ for each $i \geq 0$. So $z$ and $z^{\prime}$ are $f$-identifiable, a contradiction with the fact that $(T, A, f)$ is a canonical model.

\section{Reduction of Monotone Models}

When the Markov graph of a canonical model $(T, A, f)$ contains external loops, we can calculate the set of periods of $f$ by means of Lemma 4.6 and Theorem 4.7. If the Markov graph of $(T, A, f)$ has no external loops, we will perform the strategy described in Sec. 2. This is done in Theorem 6.7, where we construct a sequence of partial reductions associated to the model $(T, A, f)$. The proof of this theorem depends strongly on the notion of twist model and makes use of Propositions 6.4 and 6.5.

Let $(T, A, f)$ be a $y$-expansive model. We will say that $(T, A, f)$ is twist around $y$ if $f\left(Z_{i}\right) \cap Z^{\star}=\emptyset$ for $i \in\left\{1,2, \ldots, n^{\star}\right\}$. Otherwise we will say that $(T, A, f)$ is non-twist around $y$.

Note that if $(T, A, f)$ is twist around $y$ and $p$ is a type of $A^{y}$ then, from the definition of a type and the $A^{y}$-monotonicity of $f$, it follows that $f\left(Z_{i}\right) \subset$ $Z_{i+1 \bmod p}$ for each $1 \leq i \leq p$. Since $A^{y}$ contains the set of vertices of $T, \mathrm{Cl}\left(Z^{\star}\right)$ is a star whose set of endpoints contains $\left\{x_{1}, x_{2}, \ldots, x_{p}\right\}$, and a unique $y$-branch hangs from each of these endpoints. This rotational behavior of $f$ around the fixed point $y$ justifies the terminology of a twist model around $y$.

Remark 6.1. When $(T, A, f)$ is twist around a fixed point $y$, each $A^{y}$-basic interval contained in a $y$ branch does not $f$-cover any typical interval. Consequently, there cannot exist external loops in the Markov $f$-graph of $A^{y}$. That is, $\mathcal{E}(T, A, f)=\emptyset$.

Given an orbital $y$-expansive model $(T, A, f)$, by definition, there is at most one $y$-branch containing no points of $A^{\circ}$. Such a $y$-branch (if it exists) contains exactly one endpoint of $T$ and so it is an interval. We will call it the residual branch. From now on, the number of $y$-branches containing points of $A^{\circ}$ will be denoted by $n^{\circ}$. 
Remark 6.2. Let $(T, A, f)$ be an orbital $y$-expansive model. By definition, $A$ does not contain fixed points and thus $y \notin A$. Furthermore, since $(T, A, f)$ is a monotone model, $\operatorname{En}(T) \subset A$. Therefore, $y \notin \operatorname{En}(T)$ and it follows that $n^{\star} \geq 2$. On the other hand, from the fact that $(T, A, f)$ is orbital we have that $n^{\star}$ is either $n^{\circ}$ or $n^{\circ}+1$, and $n^{\star}=n^{\circ}+1$ if and only if there exists a residual branch. In summary, we have:

(i) $n^{\star} \geq 2$.

(ii) $n^{\star} \in\left\{n^{\circ}, n^{\circ}+1\right\}$, and there exists a residual branch if and only if $n^{\star}=n^{\circ}+1$.

The next lemma establishes some properties of the type of $A^{y}$ when $(T, A, f)$ is a twist model around $y$.

Lemma 6.3. Let $(T, A, f)$ be a y-expansive orbital model which is twist around $y$. Then $A^{y}$ has a unique type and it coincides with $n^{\circ}$.

Proof. Assume that $X\left(A^{y}\right)$ contains two different periodic orbits of $\Phi_{A^{y}}$ of periods $p$ and $q$. Then, since $(T, A, f)$ is twist around $y$, there exist two subsets $\mathcal{Z}=\left\{Z_{1}, Z_{2}, \ldots, Z_{p}\right\}$ and $\mathcal{W}=$ $\left\{W_{1}, W_{2}, \ldots, W_{q}\right\}$ of the set of $y$-branches such that $\mathcal{Z} \cap \mathcal{W}=\emptyset, f\left(Z_{i}\right) \subset Z_{i+1} \bmod p$ for $i=1,2, \ldots, p$ and $f\left(W_{i}\right) \subset W_{i+1} \bmod q$ for $i=1,2, \ldots, q$. Furthermore, by the definition of the $y$-branches we have that $Z_{i} \cap W_{j}=\emptyset$ for $1 \leq i \leq p$ and $1 \leq j \leq q$. Let $z \in A \cap Z_{p}$. Then $f^{i}(z) \in Z_{i \bmod p}$ for every $i \geq 0$. Since $(T, A, f)$ is orbital, there is a $k \geq 0$ such that $f^{k}(z) \in A^{\circ}$. Consequently, $A^{\circ} \subset$ $Z_{1} \cup Z_{2} \cup \ldots \cup Z_{p}$. But analogously, by taking some $w \in A \cap W_{q}$, we get that $A^{\circ} \subset W_{1} \cup W_{2} \cup \ldots \cup W_{q}$, a contradiction.

Let $P$ be the the unique periodic orbit of $\Phi_{A^{y}}$ and let $p=|P|$. Then $p \leq n^{\star}$. By Remark 6.2, $n^{\star} \in$ $\left\{n^{\circ}, n^{\circ}+1\right\}$. Now we claim that $p \leq n^{\circ}$. Indeed, assume that $p=n^{\star}=n^{\circ}+1$. Then there is one residual branch $S$ and the unique point $z$ of $X\left(A^{y}\right) \cap$ $S$ belongs to $P$. By Remark 6.2, $p \geq 2$. Therefore, there exists another $y$-branch $S^{\prime}$ such that if $z^{\prime}$ is the only point of $X\left(A^{y}\right) \cap S^{\prime}$ then $\Phi_{A^{y}}\left(z^{\prime}\right)=z$. In other words, $f\left(z^{\prime}\right) \in S$. Therefore, since $(T, A, f)$ is twist around $y, f\left(S^{\prime}\right) \subset S$. In particular, $f\left(A^{\circ} \cap S^{\prime}\right) \subset S$, in contradiction with the fact that $S$ is the residual branch. This proves the claim.

To prove $n^{\circ}=p$ we must see that $n^{\circ} \leq p$. It is enough to show that, given a $y$-branch $S$ such that $S \cap A^{\circ} \neq \emptyset$, then $z \in P$ where $z$ is the unique point of $X\left(A^{y}\right) \cap S$. On the contrary, since $P$ is the unique periodic orbit of $\Phi_{A^{y}}, \Phi_{A^{y}}^{i}(z) \neq z$ for all $i>0$. Since $(T, A, f)$ is twist around $y$, it follows that $f^{i}(S) \cap S=\emptyset$ for all $i>0$. Take $z^{\prime} \in A^{\circ} \cap S$. Then $f^{\left|A^{\circ}\right|}\left(z^{\prime}\right)=z^{\prime} \in S$, a contradiction.

Proposition 6.4. Let $(T, A, f)$ be an n-orbital canonical model which is twist around a fixed point $y$ and let $p$ be the type of $A^{y}$. Then there exist a $y$-branch $S$ and a finite set $B \subset S$ such that the following properties hold for $g=\left.f^{p}\right|_{S}$ :

(a) $(S, B, g)$ is a canonical model.

(b) $B$ contains a unique periodic orbit of $g$. Furthermore, $B^{\circ}=A^{\circ} \cap S$ and $\left|B^{\circ}\right|=\left|A^{\circ}\right| / p$.

(c) If $\left|B^{\circ}\right|>1$ then $(S, B, g)$ is $(n+1)$-orbital if $n \in\{0,1\}$ and $n$-orbital if $n \geq 2$.

(d) $\operatorname{Per}(f) \supset p \cdot \operatorname{Per}(g)$.

(e) If $p=1$ then $A^{\circ} \subset B \varsubsetneqq A$.

Proof. By Lemma 6.3 and Remark 6.2 we have $p=$ $n^{\circ}, n^{\star} \geq 2$ and $n^{\star} \in\left\{n^{\circ}, n^{\circ}+1\right\}$. Since $\mid \operatorname{En}(T) \backslash$ $A^{\circ} \mid \leq 1$ and $n^{\star} \geq 2$, we can choose $S$ to be a $y$ branch such that $\operatorname{En}(T) \cap S \subset A^{\circ}$. Without loss of generality, we can assume that $S=Z_{1}$.

In order to prove (d) it is enough to see that each $k$-periodic point of $g$ is a $k p$-periodic point of $f$. This is a direct consequence of the fact that $(T, A, f)$ is twist around $y$ and the definitions of $g$ and $S$.

Now we prove the other statements when $p=$ $n^{\circ}=1$. In this case, there are two $y$-branches: $S$ and the residual one. Moreover, $A^{\circ} \subset S$. Since $(T, A, f)$ is twist around $y, f(S) \subset S$. We take $B=$ $A \cap S$. Thus $A^{\circ}$ is the only periodic orbit contained in $B$, and (b) and (e) hold. Since $\operatorname{En}(T) \cap S \subset$ $A^{\circ}$, the only endpoint of $S$ which possibly does not belong to $A^{\circ}$ is $x_{1}$. Hence $\left|\operatorname{En}(S) \backslash A^{\circ}\right| \leq 1$. It is obvious that $(S, B, g)$ is $n$-orbital and thus (c) is satisfied. Finally, it is not difficult to prove that $(S, B, g)$ is a canonical model. Thus (a) holds and we are done in this case.

Now we consider the case $p=n^{\circ} \geq 2$. Observe that the set $f^{-p}(A) \cap S$ is not necessarily finite, but the $A$-monotonicity of $f$ implies that it has finitely many connected components, each of them being either a point or a subtree on which $f^{p}$ is constant. Note that $A \cap S \subset f^{-p}(A) \cap S$. Then we construct 
the set $B$ by taking all the points of $A \cap S$ and all vertices $V(K)$ for each connected component $K$ of $f^{-p}(A) \cap S$. Thus $B$ is finite and $A \cap S \subset B$.

Since $A^{\circ}$ is a periodic orbit and $(T, A, f)$ is twist around $y$, we get that $\left|A^{\circ} \cap Z_{i}\right|=\left|A^{\circ}\right| / p$ for $i \in\{1,2, \ldots, p\}$. Moreover, $g(S) \subset S, A^{\circ} \cap S$ is a periodic orbit of $g$ of period $\left|A^{\circ}\right| / p$ and $B \subset f^{-p}(A \cap$ $S) \cap S=g^{-1}(A \cap S)$. Thus $g(B) \subset A \cap S \subset B$ and hence $B$ is $g$-invariant. On the other hand, $A^{\circ} \cap S$ is the only periodic orbit of $g$ contained in $B$. Therefore, $B^{\circ}=A^{\circ} \cap S$ and (b) holds.

Next we prove (c). Assume that $\left|B^{\circ}\right|>1$. Since $\operatorname{En}(T) \cap S \subset A^{\circ}$, the only element of $\operatorname{En}(S)$ which possibly does not belong to $B^{\circ}$ is $x_{1}$, and so we have that $\left|\operatorname{En}(S) \backslash B^{\circ}\right| \leq 1$. To finish the proof of (c) we claim that for each $x \in B, g^{n+1}(x) \in B^{\circ}$ if $n \in\{0,1\}$ and $g^{n}(x) \in B^{\circ}$ if $n \geq 2$. To prove the claim, set $n=p q+r$ with $q \geq 0$ and $0 \leq r<p$. Since $x \in B, f^{p}(x) \in A \cap S$. Therefore, since $S=Z_{1}$ and $(T, A, f)$ is $n$-orbital and twist, we have that $f^{n}\left(f^{p}(x)\right)=f^{n+p}(x)=f^{(q+1) p+r}(x) \in A^{\circ} \cap Z_{r+1}$. Hence, for $i \geq 0$ we have

$$
f^{n+p+i}(x) \in A^{\circ} \cap Z_{r+1+i} \bmod p .
$$

When $n=0$ we have $r=0$, and by taking $i=0$ in (2) we get that $g(x)=f^{p}(x) \in A^{\circ} \cap Z_{1}=B^{\circ}$. If $n=1$, since $p>1$ we have $q=0$ and $r=1$. Then, by taking $i=p-1$ in (2) we get that $g^{2}(x)=$ $f^{2 p}(x) \in A^{\circ} \cap Z_{1}=B^{\circ}$. Finally, when $n \geq 2$ we take $i=p n-n-p$. Since $p \geq 2$ and $n \geq 2$, it follows that $i \geq 0$. Then from (2) we obtain that $g^{n}(x)=f^{p n}(x) \in A^{\circ} \cap Z_{r+1+(p n-p q-r-p) \bmod p}=$ $A^{\circ} \cap Z_{1}=B^{\circ}$. This ends the proof of the claim, and hence (c) follows.

Finally we must prove (a), i.e. that $(S, B, g)$ is a canonical model. First we will show that $g$ is $B$-monotone. Let $[x, z]$ be an interval such that $[x, z] \cap B=\{x, z\}$. Since $g=f^{p}$, we must see that $f^{p}([x, z])=\left[f^{p}(x), f^{p}(z)\right]$ and $\left.f^{p}\right|_{[x, z]}$ is monotone. From the definition of $B$, we have that either $[x, z]$ is contained in a connected component of $f^{-p}(A) \cap S$ and thus $f^{p}([x, z])$ reduces to a point of $A$, or $(x, z) \cap f^{-p}(A)=\emptyset$. In the first case it is obvious that $f^{p}([x, z])=\left[f^{p}(x), f^{p}(z)\right]$ and $\left.f^{p}\right|_{[x, z]}$ is monotone. Now assume that $(x, z) \cap f^{-p}(A)=\emptyset$. Since $A$ is $f$-invariant, $(x, z) \cap f^{-i}(A)=\emptyset$ for $0 \leq i<p$. Since $(x, z) \cap A=\emptyset$ and $\operatorname{En}(T) \subset A$, there exists a minimal interval in $T$ (with respect to the inclusion relation) containing $[x, z]$ whose endpoints belong to $A$. Since $f$ is $A$-monotone we have that $\left.f\right|_{[x, z]}$ is monotone. In particular, $f([x, z])=[f(x), f(z)]$. Moreover, $(f(x), f(z)) \cap A=\emptyset$, since otherwise $(x, z) \cap f^{-1}(A) \neq \emptyset$, a contradiction. In the same way, it can be proved inductively that $\left.f^{i}\right|_{[x, z]}$ is monotone for each $1<i \leq p$. Therefore, $g$ is $B$ monotone.

In order to complete the proof we must show that there are no $g$-identifiable vertices. On the contrary, assume that there exist $v_{1}, v_{2} \in V(S) \backslash B$ that are $g$-identifiable. Since the only possible point of $V(S) \backslash V(T)$ is the unique point of $X\left(A^{y}\right) \cap S$, which belongs to $B$, we have that $v_{1}, v_{2} \in V(T)$. We consider two cases.

In the first case we assume that $\left[g^{i}\left(v_{1}\right), g^{i}\left(v_{2}\right)\right] \cap$ $B=\emptyset$ for $i \geq 0$. In other words, $\left[f^{i p}\left(v_{1}\right), f^{i p}\left(v_{2}\right)\right] \cap$ $B=\emptyset$ for $i \geq 0$. Moreover, since $g$ is $B$-monotone, we have $\left[g^{i}\left(v_{1}\right), g^{i}\left(v_{2}\right)\right]=g^{i}\left(\left[v_{1}, v_{2}\right]\right)$ for $i \geq 0$. Since $(T, A, f)$ is a canonical model, $v_{1}$ and $v_{2}$ are not $f$ identifiable. Therefore, there exists $j \geq 1$ (which we take as small as possible) such that $\left[f^{j}\left(v_{1}\right), f^{j}\left(v_{2}\right)\right] \cap$ $A \neq \emptyset$ and $f^{j}\left(v_{1}\right) \neq f^{j}\left(v_{2}\right)$. Since $f$ is $A$-monotone, $\left[f^{j}\left(v_{1}\right), f^{j}\left(v_{2}\right)\right]=f^{j}\left(\left[v_{1}, v_{2}\right]\right)$. Take $k \in \mathbb{N}$ such that $k p>j$. Since $A$ is $f$-invariant, $f^{k p}\left(\left[v_{1}, v_{2}\right]\right) \cap A \neq \emptyset$. Then $\emptyset \neq A \cap g^{k}\left(\left[v_{1}, v_{2}\right]\right)=A \cap\left[g^{k}\left(v_{1}\right), g^{k}\left(v_{2}\right)\right] \subset$ $B \cap\left[g^{k}\left(v_{1}\right), g^{k}\left(v_{2}\right)\right]$, a contradiction. This ends the proof of the proposition in this case.

Secondly, assume that there is a $j \geq 1$ such that $\left[g^{i}\left(v_{1}\right), g^{i}\left(v_{2}\right)\right] \cap B=\emptyset$ for $0 \leq i<j$ and $g^{j}\left(v_{1}\right)=$ $g^{j}\left(v_{2}\right) \in B$. In other words, $\left[f^{i p}\left(v_{1}\right), f^{i p}\left(v_{2}\right)\right] \cap B=$ $\emptyset$ for $0 \leq i<j$ and $f^{j p}\left(v_{1}\right)=f^{j p}\left(v_{2}\right) \in B$. Moreover, since $g$ is $B$-monotone, we have that $\left[f^{i p}\left(v_{1}\right), f^{i p}\left(v_{2}\right)\right]=f^{i p}\left(\left[v_{1}, v_{2}\right]\right)$ for $0 \leq i \leq j$. In particular,

$$
f^{j p}\left(\left[v_{1}, v_{2}\right]\right)=\left\{f^{j p}\left(v_{1}\right)\right\}=\left\{f^{j p}\left(v_{2}\right)\right\} .
$$

Since $\left[f^{j p-p}\left(v_{1}\right), f^{j p-p}\left(v_{2}\right)\right] \cap B=\emptyset$, from the definition of $B$ it follows that $\left[f^{j p-p}\left(v_{1}\right), f^{j p-p}\left(v_{2}\right)\right]$ does not intersect any connected component of $f^{-p}(A) \cap S$. Thus

$$
f^{j p}\left(v_{1}\right)=f^{j p}\left(v_{2}\right) \in B \backslash A .
$$

Since $(T, A, f)$ is a canonical model, $v_{1}$ and $v_{2}$ are not $f$-identifiable. Therefore, there exists some $k \geq 1$ (which we take as small as possible) such that $\left[f^{k}\left(v_{1}\right), f^{k}\left(v_{2}\right)\right] \cap A \neq \emptyset$ and $f^{k}\left(v_{1}\right) \neq$ $f^{k}\left(v_{2}\right)$. From (4) it follows that $k<j p$. Since $f$ is $A$-monotone, $\left[f^{k}\left(v_{1}\right), f^{k}\left(v_{2}\right)\right]=f^{k}\left(\left[v_{1}, v_{2}\right]\right)$. Then, since $\left[f^{k}\left(v_{1}\right), f^{k}\left(v_{2}\right)\right] \cap A \neq \emptyset$, we have that 
$\emptyset \neq f^{j p-k}\left(\left[f^{k}\left(v_{1}\right), f^{k}\left(v_{2}\right)\right]\right) \cap A=f^{j p}\left(\left[v_{1}, v_{2}\right]\right) \cap A$. Therefore, (4) and (3) are in contradiction to each other.

To compute the set of periods of a canonical model we will use a subclass of the external loops whose length satisfies certain properties. Now we establish a notation for this kind of loops. Let $(T, A, f)$ be a a $y$-expansive $n$-orbital model. Let $p$ be a type of $A^{y}$ and let $q$ be a rotation index associated to $p$. Then we define

$$
\begin{gathered}
\tilde{\mathcal{E}}(T, A, f)=\{\beta \in \mathcal{E}(T, A, f):|\beta| \in p \mathbb{N} \\
\left.|\beta| \leq\left|A^{\circ}\right|+p+q+n+1\right\}
\end{gathered}
$$

Proposition 6.5. Let $(T, A, f)$ be an n-orbital canonical model which is non-twist around a fixed point $y$. Let $p$ be a type of $A^{y}$ and let $q$ be a rotation index of $(T, A, f)$ associated to $p$. Then at least one of the following statements hold:

(a) There exist a tree $S \subset T$ and a finite set $B \subset$ $S$ such that $A^{\circ} \subset B \varsubsetneqq A$ and $\left(S, B,\left.f\right|_{S}\right)$ is an $n$-orbital canonical model;

(b) $\tilde{\mathcal{E}}(T, A, f) \neq \emptyset$.

In particular, (b) holds if $\operatorname{En}(T) \subset A^{\circ}$

Proof. Let $W$ be the set of points $z \in A^{y}$ that satisfy the following two properties:

(i) There exists $N \in\{1,2, \ldots, p\}$ such that $z \in$ $Z_{N}$ but $f(z) \notin Z_{N+1} \bmod p$

(ii) There exist $z^{\prime} \in A^{y} \backslash\{z\}$ and $w \in A^{\circ}$ such that $z^{\prime} \preceq w$ and $z \preceq f\left(z^{\prime}\right)$.

A sufficient condition for (ii) is the following property:

(ii') There exists $z^{\prime \prime} \in A^{\circ}$ such that $z \preceq z^{\prime \prime}$.

To see it, take $w=z^{\prime}$ as the unique point of $f^{-1}\left(z^{\prime \prime}\right) \cap A^{\circ}$ when $\{z\} \neq f^{-1}\left(z^{\prime \prime}\right) \cap A^{\circ}$, and take $w=z^{\prime}$ as the unique point of $f^{-1}(z) \cap A^{\circ}$ otherwise.

We start by claiming that if $\operatorname{En}(T) \subset A^{\circ}$ then $W \neq \emptyset$. To prove the claim assume that $W=\emptyset$. Since in this case (ii') holds for every $z \in A^{y}$, we see that (i) does not hold for any $z \in Z\left(A^{y}\right)$. Thus $f\left(A^{y} \cap Z_{i}\right) \subset Z_{i+1} \bmod p$ for $i=1,2, \ldots, p$. Then, by the $A^{y}$-monotonicity of $f$, we have $f\left(Z_{i}\right) \subset$ $Z_{i+1 \bmod p}$ for $i=1,2, \ldots, p$. Since $A^{\circ}$ is a periodic orbit and $\operatorname{En}(T) \subset A^{\circ}$, we easily get that $n^{\star}=n^{\circ}=p$. Therefore $f\left(Z_{i}\right) \cap Z^{\star}=\emptyset$ for $i=1,2, \ldots, n^{\star}$ and so $(T, A, f)$ is twist around $y$, in contradiction with the hypotheses. This proves the claim.

To prove the proposition we consider two cases. First we assume that $W \neq \emptyset$ and we prove that (b) holds. In the proof of this case, the subindexes will be considered modulo $p$. Let $k \in\{1,2, \ldots, p\}$ be such that $q=q_{k}$. By the $A$-monotonicity of $f$ and the definition of $q, f\left(\left[y, f^{i-1}\left(x_{k}\right)\right]\right)=\left[y, f^{i}\left(x_{k}\right)\right]$ for $1 \leq i \leq q$ and $\left[y, f^{q}\left(x_{k}\right)\right] \cap A \neq \emptyset$. We have $f^{q}\left(x_{k}\right) \in Z_{k+q}$. This is obvious if $q=0$ and it follows from Lemma 4.4 if $q>0$.

Let $a \in\left[y, f^{q}\left(x_{k}\right)\right] \cap A$. Take $z \in W$ and let $N \in\{1,2, \ldots, p\}, w \in A^{\circ}$ and $z^{\prime} \in A^{y} \backslash\{z\}$ be such that $z \in Z_{N}, f(z) \notin Z_{N+1}, z^{\prime} \preceq w$ and $z \preceq f\left(z^{\prime}\right)$. Since $(T, A, f)$ is $n$-orbital and $w \in A^{\circ}$, there exists $s \leq n+\left|A^{\circ}\right|-1$ such that $f^{s}(a)=w$. Thus $z^{\prime} \preceq$ $f^{s}(a)$. If

$$
f^{i}\left(\left[y, f^{q}\left(x_{k}\right)\right]\right) \subset\left[y, x_{k+q+i}\right] \cup Z_{k+q+i}
$$

is satisfied for each $0 \leq i \leq s+1$, then we have that $z^{\prime} \in f^{s}\left(\left[y, f^{q}\left(x_{k}\right)\right]\right), z \in f^{s+1}\left(\left[y, f^{q}\left(x_{k}\right)\right]\right)$, $Z_{N}=Z_{k+q+s+1}$ and $f(z) \notin Z_{k+q+s+2}$. Summarizing, there exists a minimum non-negative integer $t \leq s+1 \leq n+\left|A^{\circ}\right|$ such that (5) holds for each $0 \leq i \leq t$ and $f^{t}\left(\left[y, f^{q}\left(x_{k}\right)\right]\right)$ contains a point $u$ whose image does not belong to $Z_{k+q+t+1}$.

Since $f\left(x_{k+q+t}\right) \in Z_{k+q+t+1}$, there exists a $A^{y}$ basic interval $L=[b, c] \subset\left[x_{k+q+t}, u\right]$ such that $f(b) \in Z_{k+q+t+1}$ and $f(c) \notin Z_{k+q+t+1}$. Then $L$ $f$-covers $\left[y, x_{k+q+t+1}\right]=I_{k+q+t+1}$. By using $q+t$ times Lemma 3.1 by backwards induction we obtain the following loop $\beta$ in the $f$-graph of $A^{y}$ :

$$
\begin{aligned}
I_{k} \rightarrow J^{1} & \rightarrow J^{2} \rightarrow \ldots \rightarrow J^{q+t-1} \rightarrow L \\
& \rightarrow I_{k+q+t+1} \rightarrow I_{k+q+t+2} \rightarrow \ldots \rightarrow I_{k}
\end{aligned}
$$

where $J^{i}$ is a $A^{y}$-basic interval contained in $f^{i}\left(I_{k}\right)$ for each $1 \leq i \leq q+t-1$. Since $L$ is not a typical interval, $\beta$ is an external loop, and $|\beta|=q+t+$ $1+p-(q+t+1 \bmod p) \in p \mathbb{N}$. Observe that $|\beta| \leq q+t+1+p \leq q+n+\left|A^{\circ}\right|+1+p$. Hence, (b) holds when $W \neq \emptyset$ and, in particular, when $\operatorname{En}(T) \subset A^{\circ}$.

From now on we assume that $W=\emptyset$. From the above claim, $\operatorname{En}(T) \not \subset A^{\circ}$ and thus $\left|\operatorname{En}(T) \backslash A^{\circ}\right|=$ 1. Hence, there is a unique $y$-branch containing some endpoint which does not belong to $A^{\circ}$. By Remark 6.2, $n^{\star} \geq 2$ and $n^{\star} \in\left\{n^{\circ}, n^{\circ}+1\right\}$. We also recall that the $y$-branches are labeled in such a way 
that $f\left(x_{i}\right) \in Z_{i+1} \bmod p$ for $i=1,2, \ldots, p$. We shall consider the following cases:

Case 1. $p=1$.

Assume that $Z_{1} \cap A^{\circ} \neq \emptyset$. Each $z \in Z_{1} \cap A^{\circ}$ verifies (ii') and, since $W=\emptyset$, it does not verify (i). In consequence, $A^{\circ} \subset Z_{1}$ and $n^{\circ}=1$. Since $n^{\star} \in\left\{n^{\circ}, n^{\circ}+1\right\}$ and $n^{\star} \geq 2$, it follows that $n^{\star}=2$. Therefore $Z_{2}$ is the residual branch and $\operatorname{En}(T) \cap Z_{1} \subset A^{\circ}$. In particular, each point in $Z_{1}$ verifies (ii'). Since $W=\emptyset$, no point in $Z_{1} \cap A^{y}$ verifies (i) and thus $f\left(Z_{1} \cap A^{y}\right) \subset Z_{1}$. Since $f$ is $A^{y}$-monotone, it follows that $f\left(Z_{1}\right) \subset Z_{1}$. We set $S=Z_{1}$ and $B=A \cap S$. Then $B \varsubsetneqq A$. It is not difficult to prove that $\left(S, B,\left.f\right|_{S}\right)$ is an $n$-orbital canonical model. Therefore (a) holds and we are done in this case.

Now suppose that $Z_{1} \cap A^{\circ}=\emptyset$. Then, from the fact that $(T, A, f)$ is $n$-orbital, it follows that $f^{r}\left(x_{1}\right) \notin Z_{1}$ for some $r \leq n$ (which we take as small as possible). By the definition of type, $f\left(x_{1}\right) \in Z_{1}$ and hence $I_{1} f$-covers $\left[x_{1}, f\left(x_{1}\right)\right]$. Also $\left[f^{i-1}\left(x_{1}\right), f^{i}\left(x_{1}\right)\right] f$-covers $\left[f^{i}\left(x_{1}\right), f^{i+1}\left(x_{1}\right)\right]$ for $1 \leq$ $i \leq r-2$ and $\left[f^{r-2}\left(x_{1}\right), f^{r-1}\left(x_{1}\right)\right] f$-covers $I_{1} \subset$ $\left[f^{r-1}\left(x_{1}\right), f^{r}\left(x_{1}\right)\right]$. By using Lemma 3.1 by backwards induction, as above we obtain a loop in the $f$-graph of $A^{y}$ of length $r \leq n$. Since $\left[x_{1}, f\left(x_{1}\right)\right]$ does not contain typical intervals, this loop is external. Hence (b) holds in this case.

Case 2. $p>1$ and $n^{\star}=n^{\circ}+1$.

In this case there is a residual branch $Z_{i}$ for some $i \in$ $\left\{1,2, \ldots, n^{\star}\right\}$. We claim that $i>p$. Indeed, if $i \leq p$ then, since $p>1$, it follows that $i-1(\bmod p) \neq i$. Hence, $Z_{i-1} \bmod p$ is not residual. Since $\mid \operatorname{En}(T) \backslash$ $A^{\circ} \mid=1$, it follows that $Z_{i-1} \bmod p \cap \operatorname{En}(T) \subset A^{\circ}$. Thus each point in $A^{y} \cap Z_{i-1}$ mod $p$ verifies (ii') from the definition of $W$. On the other hand, each point $z \in A^{\circ} \cap Z_{i-1} \bmod p \subset A^{y} \cap Z_{i-1} \bmod p$ verifies $f(z) \notin$

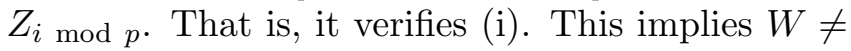
$\emptyset$, a contradiction. This proves the claim.

From above, it follows that $\operatorname{En}(T) \cap Z_{i} \subset A^{\circ}$ for $i=1,2, \ldots, p$. Therefore, each $z \in A^{y} \cap Z_{i}$ satisfies (ii') from the definition of $W$ and, since $W=\emptyset$, these points do not satisfy (i). Consequently, $f\left(A^{y} \cap Z_{i}\right) \subset Z_{i+1} \bmod p, n^{\circ}=p$ and $Z_{n^{\star}}=Z_{n^{\circ}+1}$ is the residual branch. By the $A^{y}$ monotonicity, $f\left(Z_{i}\right) \subset Z_{i+1} \bmod p$ and $f\left(\left[y, x_{i}\right]\right) \subset$ $\left[y, x_{i+1} \bmod p\right] \cup Z_{i+1} \bmod p$ for $i=1,2, \ldots, p$. Thus, if we define $S=\left\langle A^{\circ}\right\rangle_{T}=T \backslash\left(Z_{n^{\star}} \cup\left(y, x_{n^{\star}}\right]\right)$, then $f(S) \subset S$. Finally, if we define $B=A \cap S$ then $B \varsubsetneqq A$ and it is not difficult to prove that $\left(S, B,\left.f\right|_{S}\right)$ is an $n$-orbital canonical model. Hence (a) holds and we are done.

Case 3. $p>1$ and $n^{\star}=n^{\circ}$.

In this case, $A^{\circ} \cap Z_{i} \neq \emptyset$ for each $1 \leq i \leq n^{\star}$. We claim that, for some $N \in\{1,2, \ldots, p\}$, there exists $a \in A^{y} \cap Z_{N}$ such that $f(a) \notin Z_{N+1}$ mod $p$. Indeed, when $p=n^{\star}$ the claim follows since $(T, A, f)$ is nontwist around $y$. To end the proof of the claim we assume that $p<n^{\star}$ and $f\left(A^{y} \cap Z_{i}\right) \subset Z_{i+1} \bmod p$ for $i=1,2, \ldots, p$. Then $A^{\circ} \subset Z_{1} \cup Z_{2} \cup \ldots \cup Z_{p}$ and $Z_{n^{\star}} \cap A^{\circ}=\emptyset$, a contradiction. Thus the claim follows.

Since $a$ satisfies (i) from the definition of $W$ and $W=\emptyset$ we have:

$$
\left\{x \in A^{\circ}: x \succeq a\right\}=\emptyset .
$$

Therefore, since $\left|\operatorname{En}(T) \backslash A^{\circ}\right|=1$, the unique point $e$ in $\operatorname{En}(T) \backslash A^{\circ}$ must satisfy $a \preceq e,[a, e] \cap$ $V(T)=\{e\}$ and $[a, e] \cap A^{\circ}=\emptyset$. Since $Z_{N} \cap A^{\circ} \neq \emptyset$, there exists $v \in\left(V(T) \cup A^{\circ}\right) \cap Z_{N}$ such that $v \prec a$ and

$$
(v, e) \cap\left(V(T) \cup A^{\circ}\right)=\emptyset .
$$

Since $y \notin Z_{N}$, we get that $x \in(v, e) \cap A^{y}$ implies $x \in A \backslash A^{\circ}$. Let $z$ be the minimum (with respect to the $\prec$ ordering) of the points of $(v, a] \cap A^{y}$ such that $f(z) \notin Z_{N+1} \bmod p$ (this point exists since $f(a) \notin$ $Z_{N+1 \bmod p}$ ). We have $x_{N} \preceq v \prec z \preceq a \preceq e$ and

$$
f\left((v, z) \cap A^{y}\right) \subset Z_{N+1 \bmod p} .
$$

Set $R=T \backslash(v, e]$. Observe that $R=\left\langle A^{\circ}\right\rangle_{T} \supset$ $Z_{N+1 \bmod p}$. Clearly, for each point $z^{\prime} \in R$ there exists $w \in \operatorname{En}(T) \cap A^{\circ}$ such that $z^{\prime} \preceq w$. Consequently, if there exists $z^{\prime} \in R \cap A^{y}$ such that $f\left(z^{\prime}\right) \in[z, e]$ (that is, $z \preceq f\left(z^{\prime}\right)$ ), it follows that $z$ verifies (i) and (ii) from the definition of $W$; a contradiction since $W=\emptyset$. Therefore,

$$
f\left(R \cap A^{y}\right) \cap[z, e]=\emptyset .
$$

Furthermore, if the image of some $x \in R \cap A^{y}$ belongs to $(v, z)$, then by (8) we have that $f^{2}(x) \in$ $R \cap A^{y}$ and hence $f^{2}(x) \notin[z, e]$. This fact, together 
with (9), gives us that $f^{i}\left(R \cap A^{y}\right) \cap[z, e]=\emptyset$ for all $i \geq 0$. Since $f$ is $A^{y}$-monotone, it follows that

$$
f^{i}(R) \cap[z, e]=\emptyset \text { for } i \geq 0 .
$$

We define $S=\mathrm{Cl}\left(\bigcup_{i>0} f^{i}(R)\right)$. Since $f(R) \supset R, S$ is connected. That is, $S$ is a subtree of $T$. Clearly, $f(S) \subset S$ and $R \subset S$. Moreover, by (10), $S \subset$ $T \backslash(z, e]$. Thus there exists $v^{\prime} \in \operatorname{En}(S)$ such that $v \preceq v^{\prime} \preceq z$ and $S=T \backslash\left(v^{\prime}, e\right]$. From the definition of $S$ and from the $A^{y}$-monotonicity of $f$ we deduce immediately that $v^{\prime} \in A^{y}$ (in fact, $S=f^{\left|A^{y}\right|}(R)$ ). Hence, $v^{\prime} \in A \backslash A^{\circ}$. We define $B=A \cap S$. Observe that $A^{\circ} \subset B \varsubsetneqq A$, since at least $e$ does not belong to $B$. Clearly, $f(B) \subset B$. Moreover, it is not difficult to show that $\left(S, B,\left.f\right|_{S}\right)$ is a canonical model. This model is $n$-orbital, since $A^{\circ}$ is the unique periodic orbit contained in $B$, and $\operatorname{En}(S) \backslash\left\{v^{\prime}\right\} \subset A^{\circ}$.

In the rest of this section, we use recursively the above theorem to study the set of periods of a canonical model. To do it, we introduce the following notions.

Let $(T, A, f)$ be a canonical model and let $p \in$ $\mathbb{N}$. We say that a canonical model $\left(T^{\prime}, A^{\prime}, f^{\prime}\right)$ is a partial p-reduction of $(T, A, f)$ if $T^{\prime} \subset T, f^{\prime}=\left.f^{p}\right|_{T^{\prime}}$ and $\operatorname{Per}(f) \supset p \operatorname{Per}\left(f^{\prime}\right)$.

Let $(T, A, f)$ be a 2 -orbital canonical model. A sequence $\left\{\left(T_{i}, A_{i}, f_{i}\right), y_{i}, p_{i}\right\}_{i=1}^{m}$ will be called a $s e$ quence of partial reductions of $(T, A, f)$ if and only if:

(i) $\left(T_{1}, A_{1}, f_{1}\right)=(T, A, f)$

(ii) $\left(T_{i}, A_{i}, f_{i}\right)$ is a $y_{i}$-expansive 2-orbital canonical model for $1 \leq i<m$.

(iii) $\left(T_{i+1}, A_{i+1}, f_{i+1}\right)$ is a partial $p_{i}$-reduction of $\left(T_{i}, A_{i}, f_{i}\right)$ for $1 \leq i<m$.

(iv) $\left|A_{i}{ }^{\circ}\right|=p_{i}\left|A_{i+1}{ }^{\circ}\right|$ for $1 \leq i<m$. Moreover, $A_{i}{ }^{\circ} \subset A_{i+1} \varsubsetneqq A_{i}$ when $p_{i}=1$.

(v) $\tilde{\mathcal{E}}\left(T_{i}, A_{i}, f_{i}\right)=\emptyset$ for $1 \leq i<m$.

(vi) $\left(T_{m}, A_{m}, f_{m}\right)$ is a canonical model such that $A_{m}$ contains a unique periodic orbit and either

(vi.1) $\left|A_{m}{ }^{\circ}\right|=1$ and thus $\left(T_{m}, A_{m}, f_{m}\right)$ is a trivial model

or

(vi.2) $\left(T_{m}, A_{m}, f_{m}\right)$ is a $y_{m}$-expansive 2-orbital canonical model, $p_{m}$ is a type of $A_{m}^{y_{m}}$ and $\tilde{\mathcal{E}}\left(T_{m}, A_{m}, f_{m}\right) \neq \emptyset$.
Observe that if $m=1$ then, by (i) and (vi.2), $(T, A, f)$ is a $y_{1}$-expansive 2 -orbital model, $p_{1}$ is a type of $A^{y_{1}}$ and $\tilde{\mathcal{E}}(T, A, f) \neq \emptyset$.

Remark 6.6. Given a sequence of partial reductions $\left\{\left(T_{i}, A_{i}, f_{i}\right), y_{i}, p_{i}\right\}_{i=1}^{m}$ of $(T, A, f)$, from (iv) it follows that $|A|=p_{1} p_{2} \cdots p_{m-1}\left|A_{m}^{\circ}\right|$. Moreover, since $\operatorname{Per}\left(f_{i}\right) \supset p_{i} \operatorname{Per}\left(f_{i+1}\right) \cup\{1\}$ for $1 \leq i<m$, it follows that $\operatorname{Per}(f) \supset\left\{1, p_{1}, p_{1} p_{2}, \ldots, p_{1} p_{2} \cdots p_{m-1}\right\}$ $\cup p_{1} p_{2} \ldots p_{m-1} \operatorname{Per}\left(f_{m}\right)$.

The next theorem and corollary are the main results of this section.

Theorem 6.7. Each 2-orbital canonical model admits a sequence of partial reductions.

Proof. Let $(T, A, f)$ be a 2-orbital canonical model. During this proof, we will use the notation from the definition of a sequence of partial reductions. In particular, the roman numerals (i-vi) refer to the properties of that definition. We formally denote $\left\{\left(T_{i}, A_{i}, f_{i}\right), y_{i}, p_{i}\right\}_{i=1}^{k}$ by $\mathcal{S}^{k}$ for any $k \geq 0$ (note that $\left.\mathcal{S}^{0}=\emptyset\right)$.

We start by setting $\left(T_{1}, A_{1}, f_{1}\right)=(T, A, f)$. Therefore $\left(T_{1}, A_{1}, f_{1}\right)$ is a 2-orbital canonical model. Moreover, (i-v) hold (with 1 instead of $m$ ). Now we proceed by induction on $k$.

Let $k \geq 1$ and assume that we have constructed a sequence $\mathcal{S}^{k-1}$ and a canonical model $\left(T_{k}, A_{k}, f_{k}\right)$ such that:

(a) $A_{k}$ contains a unique periodic orbit of $f_{k}$ and $\left(T_{k}, A_{k}, f_{k}\right)$ is 2-orbital if $\left|A_{k}{ }^{\circ}\right|>1$.

(b) (i-v) hold (with $k$ instead of $m$ ).

Observe that if, in addition, there exist $y_{k}$ and $p_{k}$ such that (vi) holds (with $k$ instead of $m$ ) then $\mathcal{S}^{k}$ is a sequence of partial reductions.

Now we must define $y_{k}$ and $p_{k}$ and then decide whether $\mathcal{S}^{k}$ is a sequence of partial reductions (in this case we stop by setting $m=k$ ) or we construct a canonical model $\left(T_{k+1}, A_{k+1}, f_{k+1}\right)$ such that $\mathcal{S}^{k}$ and $\left(T_{k+1}, A_{k+1}, f_{k+1}\right)$ verify (a) and (b) (with $k+1$ instead of $k$ ).

Assume that $\left|A_{k}{ }^{\circ}\right|=1$. We set $p_{k}=1$ and define $y_{k}$ to be the unique element of $A_{k}{ }^{\circ}$. Then $\mathcal{S}^{k}$ verifies (vi.1) and thus $\mathcal{S}^{k}$ is a sequence of partial reductions. In this case we are done by setting $m=$ $k$.

Assume that $\left|A_{k}{ }^{\circ}\right|>1$. Then $\left(T_{k}, A_{k}, f_{k}\right)$ is 2- 
orbital since (a) holds. By Proposition 5.4, there exists $y_{k} \in \operatorname{Fix}\left(f_{k}\right)$ such that $\left(T_{k}, A_{k}, f_{k}\right)$ is $y_{k^{-}}$ expansive.

Let $p$ be a type of $A_{k}^{y_{k}}$. If $\tilde{\mathcal{E}}\left(T_{k}, A_{k}, f_{k}\right) \neq \emptyset$ then we define $p_{k}=p$ and (vi.2) holds (with $k$ instead of $m$ ). Hence $\mathcal{S}^{k}$ is a sequence of partial reductions and we are done by setting $m=k$.

From now on we assume that $\tilde{\mathcal{E}}\left(T_{k}, A_{k}, f_{k}\right)=\emptyset$. Since $\left|A_{k}{ }^{\circ}\right|>1,\left(T_{k}, A_{k}, f_{k}\right)$ does not verify neither (vi.1) nor (vi.2) and $\mathcal{S}^{k}$ is not a sequence of partial reductions. In order to iterate the argument we will define a model $\left(T_{k+1}, A_{k+1}, f_{k+1}\right)$ such that $\mathcal{S}^{k}$ and $\left(T_{k+1}, A_{k+1}, f_{k+1}\right)$ verify (a) and (b) with $k+1$ instead of $k$. We consider two cases.

Case 1. $\left(T_{k}, A_{k}, f_{k}\right)$ is twist around $y_{k}$.

We define $p_{k}=p$. By Proposition 6.4, there exists a $y_{k}$-branch $T_{k+1}$ and a finite set $A_{k+1} \subset$ $T_{k+1}$ such that if we define $f_{k+1}=\left.\left(f_{k}\right)^{p_{k}}\right|_{T_{k+1}}$ then $\left(T_{k+1}, A_{k+1}, f_{k+1}\right)$ is a canonical model and $\operatorname{Per}\left(f_{k}\right) \supset p_{k} \operatorname{Per}\left(f_{k+1}\right)$. Hence, $\left(T_{k+1}, A_{k+1}, f_{k+1}\right)$ is a partial $p_{k}$-reduction of $\left(T_{k}, A_{k}, f_{k}\right)$. Furthermore, $A_{k+1}$ contains a unique periodic orbit and $\left(T_{k+1}, A_{k+1}, f_{k+1}\right)$ is 2-orbital if $\left|A_{k+1}{ }^{\circ}\right|>1$. Finally,

$$
\begin{gathered}
\left|A_{k}{ }^{\circ}\right|=p_{k}\left|A_{k+1}{ }^{\circ}\right| \text { and } \\
\text { if } p_{k}=1 \text { then } A_{k}^{\circ} \subset A_{k+1} \varsubsetneqq A_{k} .
\end{gathered}
$$

Summarizing, we have constructed a canonical model $\left(T_{k+1}, A_{k+1}, f_{k+1}\right)$ in such a way that $\mathcal{S}^{k}$ and $\left(T_{k+1}, A_{k+1}, f_{k+1}\right)$ verify (a) and (b) (with $k+1$ instead of $k$ ).

Case 2. $\left(T_{k}, A_{k}, f_{k}\right)$ is non-twist around $y_{k}$.

Since $\tilde{\mathcal{E}}\left(T_{k}, A_{k}, f_{k}\right)=\emptyset$, by Proposition 6.5 there exists a tree $T_{k+1} \subset T_{k}$ and a finite set $A_{k+1} \subset$ $T_{k+1}$ such that $\left(T_{k+1}, A_{k+1},\left.f_{k}\right|_{T_{k+1}}\right)$ is a 2-orbital canonical model and

$$
A_{k}^{\circ} \subset A_{k+1} \varsubsetneqq A_{k} .
$$

We set $p_{k}=1$ and $f_{k+1}=\left.f_{k}\right|_{T_{k+1}}$. Thus $\operatorname{Per}\left(f_{k}\right) \supset$ $p_{k} \operatorname{Per}\left(f_{k+1}\right)$ and $\left(T_{k+1}, A_{k+1}, f_{k+1}\right)$ is a partial $p_{k^{-}}$ reduction of $\left(T_{k}, A_{k}, f_{k}\right)$. As above, we have constructed a canonical model $\left(T_{k+1}, A_{k+1}, f_{k+1}\right)$ such that $\mathcal{S}^{k}$ and $\left(T_{k+1}, A_{k+1}, f_{k+1}\right)$ verify (a) and (b) (with $k+1$ instead of $k$ ).

Finally we must prove that this iterative construction stops after a finite number of steps. This is a direct consequence of (11), (12) and the finiteness of $A_{1}$.

Next we will use the notion of a sequence of partial reductions to estimate the set of periods of a canonical model. A serious drawback of this notion is that it is only defined for canonical models, whereas we are interested in studying the set of periods of the more general monotone models. However, by means of Theorem 5.3, for each monotone model $(S, P, g)$ we can construct a canonical model $(T, A, f)$ associated to it (see page 15). Then we can use a sequence of partial reductions to get an estimation of $\operatorname{Per}(f)$, which differs from $\operatorname{Per}(g)$ only in finitely many periods. This motivates the following definition.

Let $(S, P, g)$ be a monotone model such that $P$ is a periodic orbit which does not consist of a fixed point. A pair $\{\mathcal{R}, K\}$, where $\mathcal{R}$ is a canonical model and $K \subset \mathbb{N}$, is said to be a complete reduction of $(S, P, g)$ if there exists a sequence of partial reductions $\left\{\left(T_{i}, A_{i}, f_{i}\right), y_{i}, p_{i}\right\}_{i=1}^{m}$ such that $K=$ $\left\{1, p_{1}, p_{1} p_{2}, \ldots, p_{1} p_{2} \cdots p_{m-1}\right\}, \mathcal{R}=\left(T_{m}, A_{m}, f_{m}\right)$ and $\left(T_{1}, A_{1}, f_{1}\right)$ is a canonical model associated to $(S, P, g)$. When $\mathcal{R}$ is non-trivial, we define the three non-negative numbers which play the central role in the characterization of $\operatorname{Per}(g)$ given by Theorem A. In this case, $p(\mathcal{R})$ will denote the type $p_{m}$ of $A_{m}^{y_{m}}$, $q(\mathcal{R})$ will denote a rotation index of $\mathcal{R}$ associated to the type $p_{m}$, and $n(\mathcal{R})$ will denote the least $n$ such that $\mathcal{R}$ is $n$-orbital. Observe that $n(\mathcal{R}) \in\{0,1,2\}$ since $\mathcal{R}$ is 2-orbital.

From this definition and Theorem 6.7 we obtain the following corollary.

Corollary 6.8. Let $(S, P, g)$ be a monotone model such that $P$ is a periodic orbit with $|P|>1$. Then $(S, P, g)$ admits a complete reduction. Given a complete reduction $\{(\bar{S}, \bar{P}, \bar{g}), K\}$ of $(S, P, g)$, there exists a (possibly empty) finite set $\mathcal{V}$ such that

$$
\operatorname{Per}(g) \supset \mathcal{V} \cup K \cup(\max K) \operatorname{Per}(\bar{g})
$$

and each element of $\mathcal{V}$ divides the least common multiple of the periods of all periodic orbits of $g$ contained in $V(S)$. Moreover, $|P|=(\max K)\left|\bar{P}^{\circ}\right|$.

Proof. Since $(S, P, g)$ is 0-orbital, by Theorem 5.3 there exists a 0 -orbital (and thus 2-orbital) canonical model $(T, A, f)$ associated to $(S, P, g)$. By Theorem $6.7,(T, A, f)$ admits a sequence of partial re- 
ductions $\left\{\left(T_{i}, A_{i}, f_{i}\right), y_{i}, p_{i}\right\}_{i=1}^{m}$. In consequence, $\left\{\left(T_{m}, A_{m}, f_{m}\right),\left\{1, p_{1}, p_{1} p_{2}, \ldots, p_{1} p_{2} \cdots p_{m-1}\right\}\right\}=$ $\{(\bar{S}, \bar{P}, \bar{g}), K\}$ is a complete reduction of $(S, P, g)$.

Since $(T, A, f)$ and $(S, P, g)$ are associated, $A$ is a periodic orbit of $f,|A|=|P|$ and there exists a (possibly empty) finite set $\mathcal{V}$ verifying the prescribed properties and such that $\operatorname{Per}(g)=\operatorname{Per}(f) \cup$ $\mathcal{V}$. By Remark 6.6, $\operatorname{Per}(f) \supset K \cup(\max K) \operatorname{Per}(\bar{g})$ and $|A|=(\max K)\left|\bar{P}^{\circ}\right|$.

\section{Proof of Theorem A}

The main results used in the proof of Theorem A are: Corollary 6.8 , which allows us to work with a complete reduction instead of the original model, and both Lemma 4.6 and Theorem 4.7 which are used to calculate the set of periods of the reduced model.

Proof of Theorem A. By the definition of a monotone model, $\operatorname{En}(S) \subset P$. Therefore, $S$ reduces to a point when $P$ consists of a fixed point, and in this case the theorem follows obviously.

Assume that $|P|>1$. The fact that there exist complete reductions of $(S, P, g)$ follows from Corollary 6.8. Moreover, given a complete reduction $\{\mathcal{R}, K\}$, we have $\operatorname{Per}(g) \supset K$. If $\mathcal{R}$ is trivial, we are done.

Assume that $\mathcal{R}$ is non-trivial and set $\mathcal{R}=$ $(\bar{S}, \bar{P}, \bar{g})$. By the definition of a complete reduction we have $\tilde{\mathcal{E}}(\mathcal{R}) \neq \emptyset$. Thus there exists $\beta \in \mathcal{E}(\mathcal{R})$ such that $|\beta| \in p \mathbb{N}$ and $|\beta| \leq\left|\bar{P}^{\circ}\right|+p+q+n+1$. We define $\lambda=|\beta| / p$. Since $\beta$ is external, by Lemma 4.6 we get $\operatorname{Per}(\bar{g}) \supset\{\lambda p i+p j: i, j \geq 1\}$. Moreover, by Lemma 4.2, $p \in \operatorname{Per}(\bar{g})$. Consequently, from Corollary 6.8 we have

$$
\begin{aligned}
\operatorname{Per}(g) & \supset K \cup\{k p\} \cup\{\lambda k p i+k p j: i, j \geq 1\} \\
& =K \cup k p \mathbb{N} \backslash\{2 k p, 3 k p, \ldots, \lambda k p\},
\end{aligned}
$$

and $|P|=k\left|\bar{P}^{\circ}\right|$. Hence, when $\left|\bar{P}^{\circ}\right| \in p \mathbb{N}$ it follows that for each $l \geq 0$ (see Remark 2.3) we have $|P|+$ $l k p=k\left(\left|\bar{P}^{\circ}\right|+l p\right) \in k p \mathbb{N}$ and therefore $\mathcal{S}_{k p}^{*}(|P|+$ $l k p)=S_{k p}(3 k p)=\{1\} \cup k p \mathbb{N}$. Hence, from (13) we have

$$
\operatorname{Per}(g) \supset K \cup \mathcal{S}_{k p}^{*}(|P|+l k p) \backslash\{2 k p, 3 k p, \ldots, \lambda k p\}
$$

and the theorem follows in this case.

Assume now that $\left|\bar{P}^{\circ}\right| \notin p \mathbb{N}$. By Theorem 4.7 we have $\operatorname{Per}(\bar{g}) \supset\left\{\left(\left|\bar{P}^{\circ}\right|+l p\right) i+p j: i, j \geq 1\right\}$ for some $0 \leq l \leq\left|\bar{P}^{\circ}\right|+q+n-1$. Then, $l \leq|P| / k+q+1$ because $n \in\{0,1,2\}$. Furthermore, if $n=0$ then, again by Theorem $4.7, l p \leq p+q-(q \bmod p)$. Thus, from Corollary 6.8 it follows that

$$
\operatorname{Per}(g) \supset\{(|P|+l k p) i+k p j: i, j \geq 1\} .
$$

Since $|P|+l k p=\left|\bar{P}^{\circ}\right| k+l k p \notin k p \mathbb{N} \backslash\{1\}$, we have

$$
\begin{gathered}
\mathcal{S}_{k p}^{*}(|P|+l k p)=S_{k p}(|P|+l k p) \\
=\{1,|P|+l k p\} \cup \\
\{(|P|+l k p) i+k p j: i \geq 0, j \geq 1\} \\
=\{1\} \cup k p \mathbb{N} \cup\{(|P|+l k p) i+k p j: i, j \geq 1\} .
\end{gathered}
$$

Hence, from (13) and (14) we have

$\operatorname{Per}(g) \supset K \cup \mathcal{S}_{k p}^{*}(|P|+l k p) \backslash\{2 k p, 3 k p, \ldots, \lambda k p\}$.

\section{Upper bounds for the type and the ro- tation index}

This section is devoted to prove the inequalities (1), from Sec. 2. In the proof of Proposition 8.1 we use Lemma 4.4 .

Proposition 8.1. Let $(T, A, f)$ be a y-expansive $n$ orbital model such that $\mathcal{E}(T, A, f) \neq \emptyset$. Let $p$ be a type of $A^{y}$ and let $q$ be a rotation index of $(T, A, f)$ associated to $p$. Then:

(a) $p \leq\left|A^{\circ}\right|+1$.

(b) If $q>0$ then $2 p+q-2 \leq\left|A^{\circ}\right|$.

If, in addition, $n=0$ then:

(c) $p \leq|A|-1$.

(d) If $p=1$ then $q+4 \leq|A|$.

(e) If $q>0$ then $2 p+q+1 \leq|A|$.

Proof. Until the end of the proof, the subindexes will be considered modulo $p$. Since $p$ is a type of $A^{y}$ we have $p \leq|\operatorname{En}(T)|$ and, since $(T, A, f)$ is orbital, there is at most 1 endpoint which does not belong to $A^{\circ}$. Therefore,

$$
|\operatorname{En}(T)| \leq\left|A^{\circ}\right|+1,
$$

which proves (a). If $n=0$ then $A=A^{\circ}$. Moreover, if $\operatorname{En}(T)=A$ then from the fact that $(T, A, f)$ is a canonical model and the unicity of canonical 
models (see Theorem B of [Alsedà et al., 1997]) we get that $T$ is a $|A|$-star whose central point is $y$ and $f([y, x])=[y, f(x)]$ for each $x \in A$. Then $\mathcal{E}(T, A, f)=\emptyset$, a contradiction. Consequently, $\operatorname{En}(T) \varsubsetneqq A$ and

$$
|\operatorname{En}(T)| \leq|A|-1 \text { when } n=0,
$$

which proves (c).

Next we prove (b). By assumption we have $q>0$. So, $x_{i} \in V(T)$ for $1 \leq i \leq p$ and, hence, for each $1 \leq i \leq p$ there are at least 2 points of $X(A)$ in $Z_{i}$ (we recall that $Z_{i}$ stands for $Z\left(A^{y}\right)_{i}$ ). Therefore,

$$
\left|X(A) \cap \bigcup_{i=1}^{p} Z_{i}\right| \geq 2 p .
$$

Let $k \in\{1,2, \ldots, p\}$ be such that $q=q_{k}$ and set $Q=\left\{f^{i}\left(x_{k}\right)\right\}_{i=0}^{q-1}$. Clearly $Q \subset V(T) \backslash A$. By Lemma 4.4, $f^{i}\left(x_{k}\right) \in Z_{k+i}$ for $0 \leq i \leq q-1$. Moreover, since $(T, A, f)$ is $y$-expansive we have that $Q$ does not contain periodic orbits and thus $|Q|=q$.

We claim that $Q \cap\left\{x_{i}\right\}_{i=1}^{p}=\left\{x_{k}\right\}$. Indeed, assume that $f^{j}\left(x_{k}\right) \in\left\{x_{i}\right\}_{i=1}^{p}$ for some $1 \leq j \leq q-$ 1. Then $f^{j}\left(x_{k}\right)=x_{k+j}$. Since $Q$ does not contain periodic orbits, $x_{k+j} \neq x_{k}$ and then we easily get that $q_{k+j}=q_{k}-j$, in contradiction with the fact that $q_{k}=q=\min \left\{q_{1}, q_{2}, \ldots, q_{p}\right\}$. Thus the claim follows.

Given $v \in Q \backslash\left\{x_{k}\right\}$, we have that $v \in V(T) \cap Z_{i}$ for some $1 \leq i \leq p, v \neq x_{i}$ and $\left[x_{i}, v\right] \cap A=\emptyset$. Since $v \in V(T)$, there exists some point $w \in X(A) \cap Z_{i}$ with $v \prec w$ which has not been taken into account in (17). Therefore,

$$
\left|X(A) \cap \bigcup_{i=1}^{p} Z_{i}\right| \geq 2 p+q-1 \text { when } q>0 .
$$

Since $|\operatorname{En}(T)| \geq|X(A)|$, (b) follows from (15) and (18).

To prove (d), assume that $p=1$. Let $a \in$ $X(A) \cap Z_{1}$. Then $f(a) \neq a$ since $(T, A, f)$ is orbital. Since $x_{1} \prec f\left(x_{1}\right)$ and $x_{1}$ belongs to the $(A \cup\{y\})$ basic interval $[y, a]$, the $(A \cup\{y\})$-monotonicity of $f$ implies that $f(a) \in Z_{1}$. By Remark 6.2, there is at least one $y$-branch different from $Z_{1}$. Therefore, since $\operatorname{En}(T) \subset A$ there exists $b \in X(A) \backslash Z_{1}$.

Now we claim that $f(b) \notin Z_{1}$. Indeed, if $f(b) \in Z_{1}$ then the $A$-monotonicity of $f$ and the fact that $(a, b) \cap A=\emptyset$ imply that $f([a, b]) \subset Z_{1}$, in contradiction with the fact that $y \in[a, b]$ and hence $y \in f([a, b])$. Thus the claim follows.

Observe that $f(b) \neq b$ since $(T, A, f)$ is orbital. Also, by the previous claim, $f(b) \notin\{a, f(a)\}$, and thus $a, b, f(a)$ and $f(b)$ are 4 different points contained in $A$. In consequence, $|A| \geq 4$. Then, (d) holds when $q=0$. When $q>0$ we have $q=q_{1}$ since $p=1$. So, by Lemma $4.4, f^{i}\left(x_{1}\right) \in\left(V(T) \cap Z_{1}\right) \backslash A$ for $0 \leq i<q$. Let $S$ be the closure of the connected component of $Z_{1} \backslash X(A)$ which contains $x_{1}$. Then $S$ is a tree whose endpoints are the elements of $X(A) \cap Z_{1}$. From the definition of $q$ it follows that, for $0<i<q, f^{i}\left(x_{1}\right)$ are vertices of $S$ which are not endpoints of $S$. Since any tree with $n$ vertices has at least $n+2$ endpoints, we get $|\operatorname{En}(S)| \geq q+1$. As we noticed above, $f(a) \in Z_{1}$ and $f(a) \neq a$ for any $a \in X(A) \cap Z_{1}$. Thus $\left|A \cap Z_{1}\right|>\left|X(A) \cap Z_{1}\right|$ and, hence, $\left|A \cap Z_{1}\right| \geq q+2$. Therefore, taking into account $b$ and $f(b)$, which are in $A$ but not in $Z_{1}$, we have $|A| \geq q+4$ and (d) holds.

To end the proof of the proposition we must show that (e) holds. So we assume that $n=0$, that is, $A$ is a periodic orbit. By (d), it is enough to consider the case $p>1$. Since $|\operatorname{En}(T)| \geq|X(A)|$, from (16) and (18) it follows that $|A| \geq 2 p+q$. So, we must show that $|A| \neq 2 p+q$.

In the rest of the proof we assume that $|A|=$ $2 p+q$ (and $q>0$ and $p>1$ ) and we will arrive to a contradiction. If $A=X(A)$, as in the proof of (c) we get that $f$ is a rigid rotation of a $|A|$-star and since $\mathcal{E}(T, A, f) \neq \emptyset$ we get that $X(A) \varsubsetneqq A$. From (18) we have

$2 p+q-1 \leq\left|X(A) \cap \bigcup_{i=1}^{p} Z_{i}\right| \leq|X(A)|<|A|=2 p+q$.

Hence, there is exactly one point $w$ in $A \backslash X(A)$ and $\left|X(A) \cap \cup_{i=1}^{p} Z_{i}\right|=2 p+q-1$.

Now we claim that $n^{\star}=p$. Indeed, assume that there exists some $y$-branch $W$ different from $Z_{1}, Z_{2}, \ldots, Z_{p}$. Since $\operatorname{En}(T) \subset A$, we have $W \cap$ $A \neq \emptyset$. Since $\left|X(A) \cap \cup_{i=1}^{p} Z_{i}\right|=2 p+q-1$ and $|A|=2 p+q$, it follows that $W \cap A=\{w\}$. Thus $w \in X(A)$, a contradiction. So the claim follows.

Let $j \in\{1,2, \ldots, p\}$ be such that $w \in Z_{j}$. We have that $Z_{j} \cap A$ is the disjoint union of $\{w\}$ and $Z_{j} \cap X(A)$, while $Z_{i} \cap A=Z_{i} \cap X(A)$ when $i \neq j$.

Since $q>0, x_{i} \notin X(A)$ for $1 \leq i \leq p$. For each point $z \in X(A) \cap Z_{i}$, we have that $[y, z]$ is a $(A \cup\{y\})$-basic interval containing $x_{i}$. Therefore, 
since $f\left(x_{i}\right) \in Z_{i+1}$ and $f$ is $(A \cup\{y\})$-monotone, we get $f\left(X(A) \cap Z_{i}\right) \subset Z_{i+1}$ for $1 \leq i \leq p$. Moreover, $f\left(X(A) \cap Z_{i}\right)=X(A) \cap Z_{i+1}$ when $i \not \equiv j$ and $i \not \equiv j+$ $1(\bmod p)$. We set $N_{i}=\left|X(A) \cap Z_{i}\right|$ for $1 \leq i \leq p$. Since $X(A) \subset A$ and $A$ is a periodic orbit, it follows that $N_{i+1}=N_{i}$ when $i \not \equiv j$ and $i \not \equiv j+1(\bmod p)$. Consequently, we have

$$
N_{j-1}=N_{j-2}=\ldots=N_{j+2}=N_{j+1} .
$$

If $f(w) \in Z_{j+1}$ then $(T, A, f)$ is twist around $y$ and, by Remark $6.1, \mathcal{E}(T, A, f)=\emptyset$, a contradiction. Therefore, $f(w) \notin Z_{j+1}$. Then $X(A) \cap Z_{j+1}=$ $f\left(X(A) \cap Z_{j}\right)$ and it follows that $N_{j+1}=N_{j}$. Thus from (19) we get that $N_{j-1}=N_{j}$. On the other hand, since $A$ is a periodic orbit, there exists a unique point $w^{\prime} \in A \cap Z_{j-1}=X(A) \cap Z_{j-1}$ such that $f\left(w^{\prime}\right)=w$. Since $w \in Z_{j} \backslash X(A)$, we get $N_{j}=N_{j-1}-1$, a contradiction.

The following result states that the inequalities (1) hold for complete reductions of monotone models.

Corollary 8.2. Let $(S, P, g)$ be a monotone model such that $P$ is a periodic orbit of $g$ with $|P|>1$. Let $\{\mathcal{R}, K\}$ be a complete reduction of $(S, P, g)$ such that $\mathcal{R}$ is non-trivial and $n(\mathcal{R})=0$. Then

$$
\begin{gathered}
p \leq r-1, \\
q+4 \leq r \text { when } p=1 \text { and } \\
2 p+q+1 \leq r \text { when } q>0,
\end{gathered}
$$

where we denote $p(\mathcal{R}), q(\mathcal{R}), n(\mathcal{R})$ and $\frac{|P|}{\max K}$ by $p$, $q, n$ and $r$ respectively.

Proof. Set $(T, A, f)=\mathcal{R}$. Since $n=0$, we have $A^{\circ}=A$. By Corollary $6.8,|A|=r$. Since $\mathcal{R}$ is nontrivial, by the definition of a complete reduction we have that $\mathcal{R}$ is a $y$-expansive 0 -orbital model for some $y \in \operatorname{Fix}(f), p$ is a type of $A^{y}, q$ is a rotation index of $\mathcal{R}$ associated to $p$ and $\tilde{\mathcal{E}}(\mathcal{R}) \neq \emptyset$. In particular, $\mathcal{E}(\mathcal{R}) \neq \emptyset$. Therefore $\mathcal{R}$ verifies the hypotheses of Proposition 8.1 and the corollary follows.

\section{Some Examples. Proof of Theorem B}

This section is devoted to prove Theorem B. In fact, we prove the following stronger result from which Theorem B can be obviously derived. Since any tree can be imbedded in $\mathbb{R}^{2}$, in what follows we will consider each tree endowed with the topology induced by the topology of $\mathbb{R}^{2}$.

Theorem 9.1. Let $K \subset \mathbb{N}$ be a set of the form $\left\{1, k_{1}, k_{2}, \ldots, k_{m}\right\}$ such that $k_{1}>1$ and $k_{i}$ strictly divides $k_{i+1}$ for $1 \leq i<m$. Set $k=k_{m}$. Then:

(a) There exists a canonical model $(R, B, h)$ such that $|B|=k$ and $\operatorname{Per}(h)=K$.

(b) Given any $r>1, p \geq 1$ and $q \geq 0$ verifying

$$
\begin{gathered}
p \leq r-1, \\
q+4 \leq r \text { when } p=1 \text { and } \\
2 p+q+1 \leq r \text { when } q>0,
\end{gathered}
$$

there exists a canonical model $(S, P, g)$ and a complete reduction $\{(\bar{S}, \bar{P}, \bar{g}), K\}$ of $(S, P, g)$ with $\left|\bar{P}^{\circ}\right|=r, p(\bar{S}, \bar{P}, \bar{g})=p, q(\bar{S}, \bar{P}, \bar{g})=q$, $n(\bar{S}, \bar{P}, \bar{g})=0$ and $\operatorname{Per}(g)=K \cup \mathcal{C}$, where $\mathcal{C}$ is a set such that

$$
\begin{aligned}
\mathcal{S}_{k p}^{*}(|P|+l k p) \backslash\{2 k p, 3 k p, \ldots, \lambda k p\} \\
\subset \mathcal{C} \subset \mathcal{S}_{k p}^{*}(|P|)
\end{aligned}
$$

with $l p=p+q-(q \bmod p)$ and $\lambda p$ being the largest multiple of $p$ smaller than $r+p+q+1$.

In order to prove Theorem 9.1 we will use the following two technical results. For Proposition 9.2 see Fig. 3, which shows an example of the construction made in that proposition.

Proposition 9.2. Let $(T, A, f)$ be a canonical model such that $A$ is a periodic orbit and let $s \geq 2$ be an integer. Then there exists a canonical model $\left(T^{\prime}, A^{\prime}, f^{\prime}\right)$ such that:

(a) There exists $y \in \operatorname{Fix}\left(f^{\prime}\right)$ such that $s$ is a type of $A^{\prime y}$ around $y$ and $\left(T^{\prime}, A^{\prime}, f^{\prime}\right)$ is $y$-expansive and twist around $y$.

(b) $A^{\prime}$ is a periodic orbit with $\left|A^{\prime}\right|=s|A|$.

(c) $(T, A, f)$ is a partial s-reduction of $\left(T^{\prime}, A^{\prime}, f^{\prime}\right)$.

(d) $\operatorname{Per}\left(f^{\prime}\right)=\{1\} \cup s \cdot \operatorname{Per}(f)$.

Proof. Set $t=|A \cup V(T)|$ and $Q^{1}=A \cup V(T)=$ $\left\{v_{1}^{1}, v_{2}^{1}, \ldots, v_{t}^{1}\right\}$ in such a way that $v_{1}^{1} \in \operatorname{En}(T)$ and $A=\left\{v_{i}^{1}\right\}_{i=1}^{|A|}$. Next we will construct $T^{\prime}$ by attaching one copy of $T$ to each endpoint of an $s$-star.

For each $2 \leq i \leq s$ we consider a tree $T^{i}$, a finite set $Q^{i}=\left\{v_{1}^{i}, v_{2}^{i}, \ldots, v_{t}^{i}\right\} \subset T^{i}$ and a homeomorphism $h_{i}: T^{i} \longrightarrow T$ such that $h_{i}\left(v_{j}^{i}\right)=v_{j}^{1}$ for each $1 \leq j \leq t$. We also set $T^{1}=T$ and $h_{1}=\left.\mathrm{Id}\right|_{\mathrm{T}^{1}}$. 


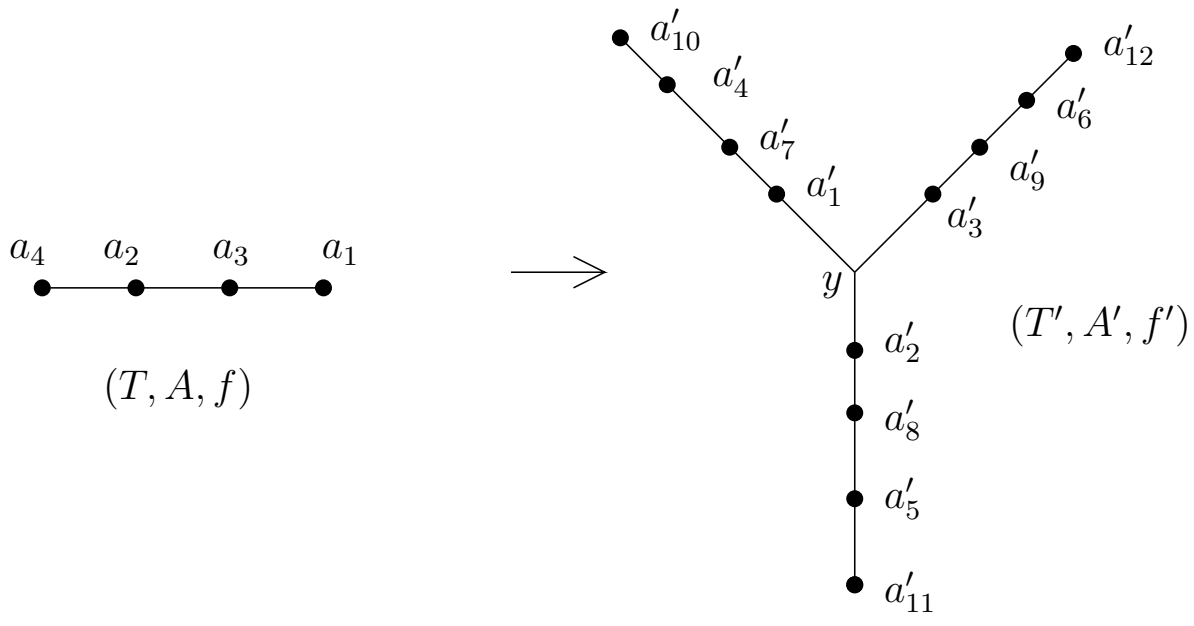

Fig. 3. An example of the construction made in Proposition 9.2, with $|A|=4$ and $s=3$. We assume $f\left(a_{i}\right)=a_{i+1} \bmod 4$ and $f^{\prime}\left(a_{i}^{\prime}\right)=a_{i+1 \bmod 12}^{\prime}$.

Now we define $T^{\prime}$ to be a tree which consists of the union of $T^{i}$ for $1 \leq i \leq s$ and an $s$-star $R$ such that $\operatorname{En}(R)=\left\{v_{1}^{1}, v_{1}^{2}, \ldots, v_{1}^{s}\right\}$. Thus $T^{\prime}$ consists of an $s$ star with one copy of $T$ attached to each endpoint. Let $y$ be the central point of $R$.

Now we are going to define the map $f^{\prime}$. First we define it on each $T^{i}$. We set $\left.f^{\prime}\right|_{T^{i}}=h_{i+1}^{-1} \circ h_{i}$ for each $1 \leq i<s$ and $\left.f^{\prime}\right|_{T^{s}}=f \circ h_{s}$. Note that $f^{\prime}$ is a homeomorphism between $T^{i}$ and $T^{i+1}$ and $f^{\prime}\left(v_{1}^{i}\right)=$ $v_{1}^{i+1}$ for each $1 \leq i<s$. Moreover, $f^{\prime}\left(v_{1}^{s}\right)=f\left(v_{1}^{1}\right) \in$ $A \cap T^{1}$.

Now we define $f^{\prime}$ on $R \backslash \operatorname{En}(R)$. We set $f^{\prime}(y)=$ $y$ and take $f^{\prime}$ to be an affine homeomorphism between $\left[y, v_{1}^{i}\right]$ and $\left[y, f\left(v_{1}^{i}\right)\right]$ for each $1 \leq i \leq s$.

Finally set $A^{\prime}=\cup_{i=1}^{s}\left\{v_{1}^{i}, v_{2}^{i}, \ldots, v_{|A|}^{i}\right\}$. Since $\left.f^{\prime}\right|_{T^{i}}$ is a homeomorphism for each $1 \leq i<s$ and $f$ is $A$-monotone, we easily get that $\left(T^{\prime}, A^{\prime}, f^{\prime}\right)$ is a monotone model. Moreover, since $(T, A, f)$ is canonical, there are no $f$-identifiable vertices in $T$. Then there are no $f^{\prime}$-identifiable vertices in $T^{\prime}$ and $\left(T^{\prime}, A^{\prime}, f^{\prime}\right)$ is a canonical model.

Now we prove (a). Set $Q=\{y\} \cup_{i=1}^{s} Q^{i}$. Clearly $A^{\prime y}=Q$ and for $1 \leq i \leq s$ we have $Z(Q)_{i}=T^{i}$ and $x(Q)_{i}=z_{i}$. Since $f^{\prime}\left(x(Q)_{i}\right)=x(Q)_{i+1}$ for $1 \leq i<$ $s$ and $f^{\prime}\left(x(Q)_{s}\right) \in Z(Q)_{1}$, it follows that $s$ is a type of $A^{\prime y}$. Moreover, $f^{\prime}\left(Z(Q)_{i}\right) \subset Z(Q)_{i+1} \bmod s$ for $1 \leq i \leq s$ and so $\left(S^{\prime}, P^{\prime}, g^{\prime}\right)$ is twist around $y$. Since there are no vertices of $T^{\prime}$ in $Z^{\star}\left(A^{\prime}\right) \backslash\{y\}$, obviously $\left(T^{\prime}, A^{\prime}, f^{\prime}\right)$ is $y$-expansive and (a) holds.

To prove the rest of the statements first we consider the case $|A|=1$. Then $T$ reduces to the unique point of $A$, that is $v_{1}^{1}$. Moreover, $T^{\prime}$ co- incides with $R$ and $A^{\prime}=\left\{v_{1}^{1}, v_{1}^{2}, \ldots, v_{1}^{s}\right\}$. Therefore $f^{\prime}$ is a rigid rotation of an $s$-star and (b), (c) and (d) follows obviously in this case.

Now we assume that $|A|>1$. We claim that

$$
f^{\prime s}(z)=f(z) \text { for each } z \in T .
$$

Indeed, take $z \in T$. We have that $f^{\prime s-1}(z)=h_{s}^{-1} \circ$ $h_{s-1} \circ h_{s-1}^{-1} \circ h_{s-2} \circ \ldots \circ h_{2} \circ h_{2}^{-1} \circ h_{1}(z)$. Since $h_{1}=\mathrm{Id}$, we get $f^{\prime s-1}(z)=h_{s}^{-1}(z) \in T^{s}$. Therefore $f^{\prime s}(z)=f^{\prime}\left(f^{\prime s-1}(z)\right)=f\left(h_{s}\left(h_{s}^{-1}(z)\right)\right)=f(z)$ and the claim follows.

Now we prove (b) and (c). Let $x \in T$ be an $n$-periodic point of $f$. Since $f^{\prime i}(x) \in T^{i+1}$ for $1 \leq$ $i<s$, from (20) it follows that $\left\{f^{\prime i}(x)\right\}_{i=0}^{s n}$ is an $s n$ periodic orbit of $f^{\prime}$. In particular, (b) holds. Thus we have $\{1\} \cup s \operatorname{Per}(f) \subset \operatorname{Per}\left(f^{\prime}\right)$. This inclusion, together with (20) and the fact that $T \subset T^{\prime}$, proves that $(T, A, f)$ is a partial $s$-reduction of $\left(T^{\prime}, A^{\prime}, f^{\prime}\right)$ and (c) holds.

Finally we prove (d). It is enough to show that $\operatorname{Per}\left(f^{\prime}\right) \subset\{1\} \cup s \operatorname{Per}(f)$. Let $P$ be an $n$-periodic orbit of $f^{\prime}$ with $n>1$. The definition of $f^{\prime}$ on $R$ implies that $y$ is a repelling fixed point of $f^{\prime s}$ on each edge of $R$. It follows that the unique periodic orbit of $f^{\prime}$ on $R$ is $\{y\}$. Therefore, $P \subset T^{\prime} \backslash R$. Moreover, since $\left(T^{\prime}, A^{\prime}, f^{\prime}\right)$ is twist we have that $n=r s$ for some $r \geq 1$ and there exists $x \in P \cap T$ such that $f^{\prime i}(x) \in T^{i+1} \bmod s$ for all $1 \leq i \leq r s$. From (20) we get that $\left\{f^{\prime i s}(x)\right\}_{i=0}^{r-1}$ is an $r$-periodic orbit of $f$. Thus $n \in s \operatorname{Per}(f)$.

By convention, a tree $T$ will be a 1-star if $T$ 


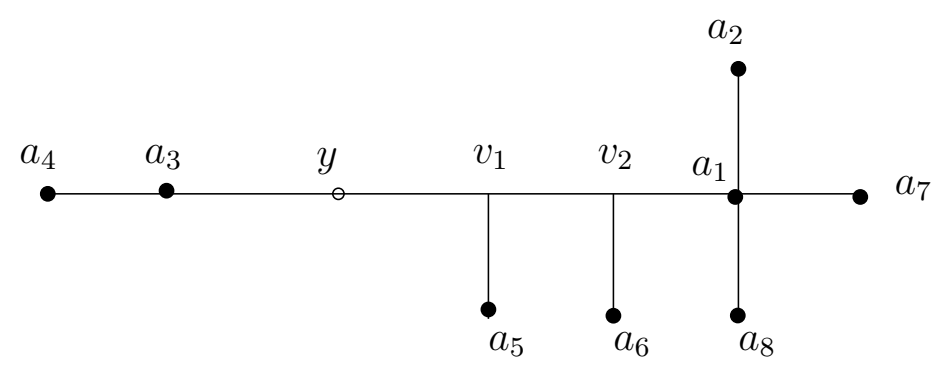

Fig. 4. A $(8,1,2)$-model

reduces to a single point, which in addition will be called the central point of $T$.

In the next proposition we will construct canonical models which exhibit prescribed sets of periods. It is the main tool for the proof of Theorem 9.1.

Proposition 9.3. Given integers $r>1, p \geq 1$ and $q \geq 0$ verifying

$$
\begin{gathered}
p \leq r-1, \\
q+4 \leq r \text { when } p=1 \text { and } \\
2 p+q+1 \leq r \text { when } q>0,
\end{gathered}
$$

there exists a monotone model $(T, A, f)$ satisfying:

(a) $(T, A, f)$ is a canonical model and $A$ is a periodic orbit with $|A|=r$.

(b) There exists $y \in \operatorname{Fix}(f)$ such that $(T, A, f)$ is $y$-expansive and non-twist around $y$.

(c) $p$ is a type of $A^{y}$ around $y$ and $q$ is a rotation index of $(T, A, f)$ associated to $p$.

(d) $\mathcal{S}_{p}^{*}(r+l p) \backslash\{2 p, 3 p, \ldots, \lambda p\} \subset \operatorname{Per}(f) \subset \mathcal{S}_{p}^{*}(r)$ with $l p=p+q-(q \bmod p)$ and $\lambda p$ being the largest multiple of $p$ smaller than $r+p+q+1$.

A canonical model which satisfies the properties (a-d) stated in Proposition 9.3 will be called an $(r, p, q)$-model.

Proof of Proposition 9.3. We are going to make the construction of $(T, A, f)$ according to four cases. In all cases, we set $A=\left\{a_{1}, a_{2}, \ldots, a_{r}\right\}$ and $f\left(a_{i}\right)=$ $a_{i+1} \bmod r$ for $i=1,2, \ldots, r$. Thus $A$ is a periodic orbit and $|A|=r$. In each case the construction will consist of two steps. First we will choose $T$ to be a tree whose endpoints are contained in $A$. We will describe $T$ by enumerating all its vertices and edges. Secondly, we will choose a point $y \in T$ to be a fixed point of $f$ and will define $f$ on each vertex of $T$.
In all cases, it is assumed that we define $f$ on the whole tree by taking an $A^{y}$-extension (recall that $A^{y}=A \cup V(T) \cup\{y\}$ ). It is not difficult to check that the images of the vertices of $T$ will be chosen in such a way $f$ is monotone on each $A$ basic interval. Therefore, by construction $f$ is $A$ monotone and $(T, A, f)$ is a monotone model.

Case 1. $p=1$.

Definition of $T$. Assume first that $q=0$. By assumption, $r \geq 4$. If $r=4$ we take $T$ as a closed interval $\left[a_{4}, a_{2}\right]$ with $a_{4}<a_{3}<a_{1}<a_{2}$. If $r>4$ then we choose $T$ to be a closed interval $\left[a_{4}, a_{r}\right]$ with $a_{4}<a_{3}<a_{1}<a_{2}<a_{5}<a_{6} \ldots<a_{r}$. We also choose a point $y \in\left(a_{3}, a_{1}\right)$.

Now assume that $q>0$. By assumption, $r \geq$ $q+4$. We choose $T$ and a point $y \in T$ such that there are two $y$-branches and:

(i) $\mathrm{Cl}\left(Z^{\star}(A)\right)$ has $q+2$ endpoints and contains the edges: $\left[y, a_{3}\right],\left[y, v_{1}\right],\left[v_{i}, v_{i+1}\right]$ for $1 \leq i \leq$ $q-1,\left[v_{q}, a_{1}\right]$ and $\left[v_{j}, a_{4+j}\right]$ for $1 \leq j \leq q$.

(ii) $Z(A)_{1}$ is a star containing the edges $\left[a_{1}, a_{2}\right]$ and $\left[a_{1}, a_{i}\right]$ for $q+5 \leq i \leq r$.

(iii) $Z(A)_{2}=\left[a_{3}, a_{4}\right]$.

See Fig. 4 for an example with $r=8$ and $q=2$.

Images of the vertices. We define $f(y)=y$. Since $V(T) \backslash A=\emptyset$ when $q=0$, we only have to consider the case $q>0$. We define $f\left(v_{i}\right)=v_{i+1}$ for $1 \leq i<q$ and $f\left(v_{q}\right)=a_{1}$.

(a) In both cases, the orbit of each vertex of $T \backslash\{y\}$ intersects $A$. Therefore, there are no $f$ identifiable vertices in $T$ and $(T, A, f)$ is a canonical model. 


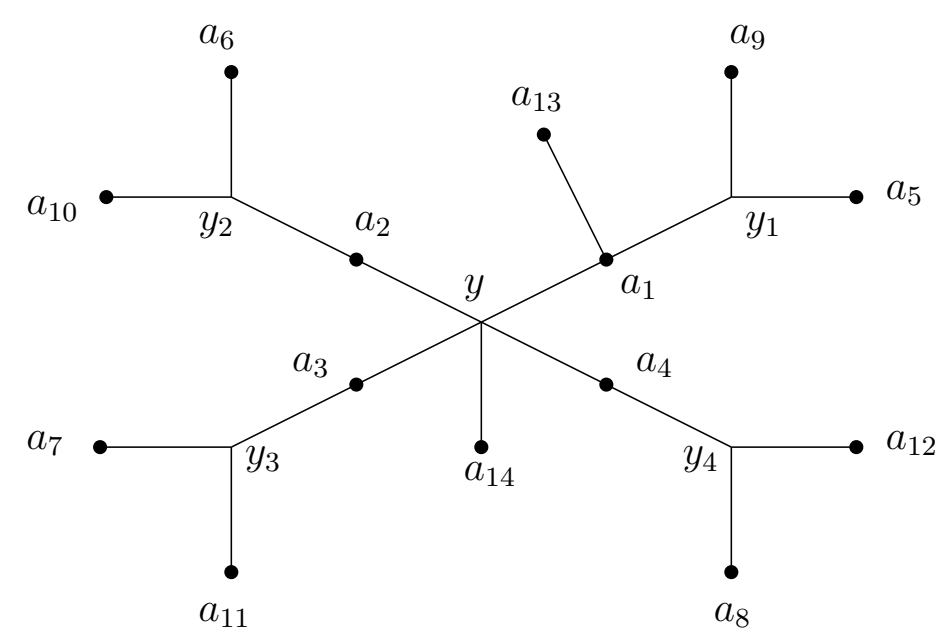

Fig. 5. A (14,4,0)-model

(b) There exists $y \in \operatorname{Fix}(f) \cap\left(a_{3}, a_{1}\right)$ and a $y$ branch $S$ such that $a_{1}, a_{2} \in S$ and $a_{3} \notin S$. Therefore $f\left(a_{1}\right) \in S, f\left(a_{2}\right) \notin S$ and $(T, A, f)$ is non-twist around $y$. Moreover, there are no periodic orbits of vertices contained in $Z^{\star}(A)$ and thus $(T, A, f)$ is $y$-expansive.

(c) In the case $q=0$, we have $x\left(A^{y}\right)_{1}=a_{1}$. When $q>0$, we have $x\left(A^{y}\right)_{1}=v_{1}$. In both cases, $f\left(x\left(A^{y}\right)_{1}\right) \succ x\left(A^{y}\right)_{1}$ and thus 1 is a type of $A^{y}$. Moreover, since $f\left(v_{i}\right)=v_{i+1}$ for $1 \leq i<q$ and $f\left(v_{q}\right)=a_{1}$, it follows that $q$ is a rotation index of the type 1 .

(d) Assume first that $q=0$. In this case, the $f$ graph of $A^{y}$ contains the loop $\left[y, a_{1}\right] \rightarrow\left[a_{1}, a_{2}\right] \rightarrow$ $\left[y, a_{1}\right]$, which is external. Thus from Lemma 4.6 we get $\operatorname{Per}(f) \supset \mathbb{N} \backslash\{2\}$. Moreover, the loop $\left[a_{1}, a_{2}\right] \rightarrow$ $\left[y, a_{1}\right] \rightarrow\left[a_{1}, a_{2}\right]$ is simple and $a_{1}$ and $a_{2}$ are not fixed points of $f^{2}$. Thus, by Lemma 3.5(a), $f$ has a point of period 2. Therefore, $\operatorname{Per}(f)=\mathbb{N}=\mathcal{S}_{p}^{*}(r)$ and (d) holds.

Assume now that $q>0$. The $f$-graph of $A^{y}$ contains the loops $\left[y, v_{1}\right] \rightarrow\left[y, v_{1}\right]$, which we take as the typical loop $\alpha$, and

$$
\begin{aligned}
{\left[v_{i}, v_{i+1}\right] \rightarrow } & {\left[v_{i+1}, v_{i+2}\right] \rightarrow \ldots } \\
& \ldots \rightarrow\left[v_{q}, a_{1}\right] \rightarrow\left[a_{1}, a_{2}\right] \rightarrow\left[v_{i}, v_{i+1}\right]
\end{aligned}
$$

which we call $\beta_{i}$ for $0 \leq i \leq q$ (where $v_{0}$ stands for $y$ ). Note that $\beta_{i}$ is negative and $\left|\beta_{i}\right|=q+$ $2-i$ for each $0 \leq i \leq q$. Since $y \in \operatorname{Fix}(f)$ and each $\beta_{i}$ satisfies (b) of Lemma 3.5, we get $\operatorname{Per}(f) \supset$
$\{1,2,3, \ldots, q+2\}$. Moreover, $\beta_{0}$ is an external loop of length $q+2$ and thus, from Lemma 4.6, we get $\operatorname{Per}(f) \supset\{q+3, q+4, \ldots\}$. Therefore, $\operatorname{Per}(f)=$ $\mathbb{N}=\mathcal{S}_{p}^{*}(r)$ and $(\mathrm{d})$ holds.

Case 2. $p>1$ and $q=0$.

Definition of $T$. Since $p<r$, we can write $r=$ $s p+k$ for some $s \geq 1$ and $0<k \leq p$. We choose $T$ and a point $y \in T$ such that there are $p y$-branches and:

(i) $\mathrm{Cl}\left(Z^{\star}(A)\right)$ is a $(p+k-1)$-star whose endpoints are $a_{1}, a_{2}, \ldots, a_{p}$ and $a_{s p+2}, a_{s p+3}, \ldots, a_{s p+k}$ and whose central point is $y$.

(ii) $Z(A)_{1}$ is the union of $\left(a_{1}, a_{s p+1}\right]$ and an $s$-star whose endpoints are $a_{1}, a_{1+p}, \ldots, a_{1+(s-1) p}$.

(iii) For each $2 \leq i \leq s, Z(A)_{i}$ is an $s$-star whose endpoints are $a_{i}, a_{i+p}, \ldots, a_{i+(s-1) p}$.

Observe that, when $s=1, Z(A)_{i}$ reduces to the point $a_{i}$ for each $2 \leq i \leq p$. When $s>1$, we denote the central point of $Z(A)_{i}$ by $y_{i}$, and the central point of $Z(A)_{1} \backslash\left(a_{1}, a_{s p+1}\right]$ by $y_{1}$. See Fig. 5 for an example with $r=14$ and $p=4$.

Images of the vertices. We define $f(y)=y$ and $f\left(y_{i}\right)=y_{i+1} \bmod p$ for each $1 \leq i \leq p$.

(a) Since there are no $A^{y}$-basic intervals with both endpoints contained in $V(T) \backslash A$, there are no $f$-identifiable vertices and thus $(T, A, f)$ is a canonical model. 


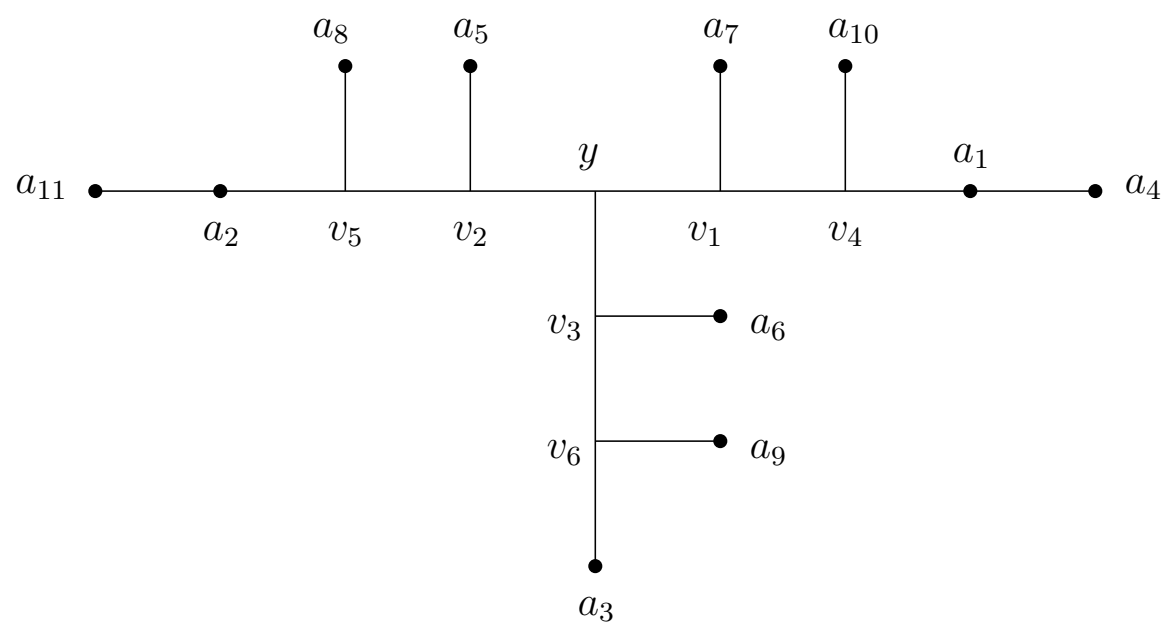

Fig. 6. An (11,3,4)-model

(b) Since $a_{1}$ and $a_{s p+1}$ are contained in $Z(A)_{1}$ and $Z^{\star}(A) \cap\left[f\left(a_{1}\right), f\left(a_{s p+1}\right)\right] \neq \emptyset$, it follows that $(T, A, f)$ is non-twist around $y$. Moreover, $(T, A, f)$ is obviously $y$-expansive since there are no vertices of $T$ in $Z^{\star}(A) \backslash\{y\}$.

(c) Observe that $\left(y, a_{i}\right) \cap A^{y}=\emptyset$ for $1 \leq i \leq p$. Therefore, $\left\{a_{i}\right\}_{i=1}^{p}=X\left(A^{y}\right)$. Since $f\left(a_{p}\right)=a_{p+1} \succ$ $a_{1}$, it follows that $p$ is a type of $A^{y}$ and the rotation index of $(T, A, f)$ associated to this type is 0 .

(d) We set $I_{i}=\left[y, a_{i}\right]$ and $K_{i}=\left[a_{i}, y_{i}\right]$ for $1 \leq i \leq p$ (recall that $K_{i}$ reduces to a single point when $s=1)$. We also set $I_{i}=\left[y_{i} \bmod p, a_{i}\right]$ for $p+1 \leq i \leq s p, I_{j}=\left[y, a_{j}\right]$ for $s p+2 \leq j \leq r$ and $I_{s p+1}=\left[a_{1}, a_{s p+1}\right]$. All these intervals are $A^{y}$-basic intervals. Moreover, the $f$-graph of $A^{y}$ contains exactly the following paths:

$$
\begin{aligned}
& I_{1} \rightarrow I_{2} \rightarrow \ldots \rightarrow I_{p} \rightarrow I_{1}, \\
& I_{1} \rightarrow I_{2} \rightarrow \ldots \rightarrow I_{r} \rightarrow I_{1}, \\
& I_{2} \rightarrow I_{3} \rightarrow \ldots \rightarrow I_{p s+1} \rightarrow I_{2},
\end{aligned}
$$

which we call $\alpha, \beta$ and $\gamma$, respectively, and

$$
\begin{aligned}
& K_{1} \rightarrow K_{2} \rightarrow \ldots \rightarrow K_{p} \rightarrow I_{p+1}, \\
& I_{p} \rightarrow K_{1}, \\
& I_{p s} \rightarrow K_{1} .
\end{aligned}
$$

We take $\alpha$ as the typical loop. Hence, $\beta$ and $\gamma$ are external loops. Since $|\beta|=r$ and $|\gamma|=s p$, from Lemma 4.6 it follows that $\operatorname{Per}(f) \supset\{i r+j p: i, j \geq$
1\} $\cup\{i s p+j p: i, j \geq 1\}$. On the other hand, $\gamma$ is negative. Thus it verifies (b) of Lemma 3.5 and, consequently, $s p \in \operatorname{Per}(f)$. Moreover, $p \in \operatorname{Per}(f)$ by Lemma 4.2. Summarizing, we have

$$
\begin{aligned}
\operatorname{Per}(f) \supset\{i r+j p: & i, j \geq 1\} \cup \\
& p \mathbb{N} \backslash\{2 p, 3 p, \ldots,(s-1) p\} .
\end{aligned}
$$

It is not difficult to check that there are no loops of other lengths. Since, by Lemma 3.2, each periodic orbit which does not intersect $A^{y}$ is associated to a loop in the $f$-graph of $A^{y}$, it follows that

$$
\begin{aligned}
\operatorname{Per}(f)=\{1, r\} \cup\{i r+j p: i, j \geq 1\} \cup \\
p \mathbb{N} \backslash\{2 p, 3 p, \ldots,(s-1) p\} .
\end{aligned}
$$

Since $q=0$, by assumption $l=0$ and $\lambda p$ is the largest multiple of $p$ smaller than $r+p+1$. Since $(s-1) p<r<r+p+1$, it follows that $(s-1) p \leq \lambda p$. When $r \notin p \mathbb{N}$, from (21) we get

$$
\operatorname{Per}(f)=\mathcal{S}_{p}^{*}(r) \backslash\{2 p, 3 p, \ldots,(s-1) p\}
$$

and thus (d) holds. On the other hand, if $r \in p \mathbb{N}$ then $\{i r+j p: i, j \geq 1\}=\{m \in p \mathbb{N}: m>r\} \subset$ $\{m \in p \mathbb{N}: m>(s-1) p\}$. Therefore (22) holds and (d) follows.

Case 3. $p>1, q>0$ and $r \notin p \mathbb{N}$.

Definition of $T$. By assumption, $r \geq 2 p+q+1$. Set $r=2 p+q+s p+k$ with $0 \leq k<p$. For simplicity, we assume $s>0$. The same construction (even simpler) can be done in the case $s=0$. The details 


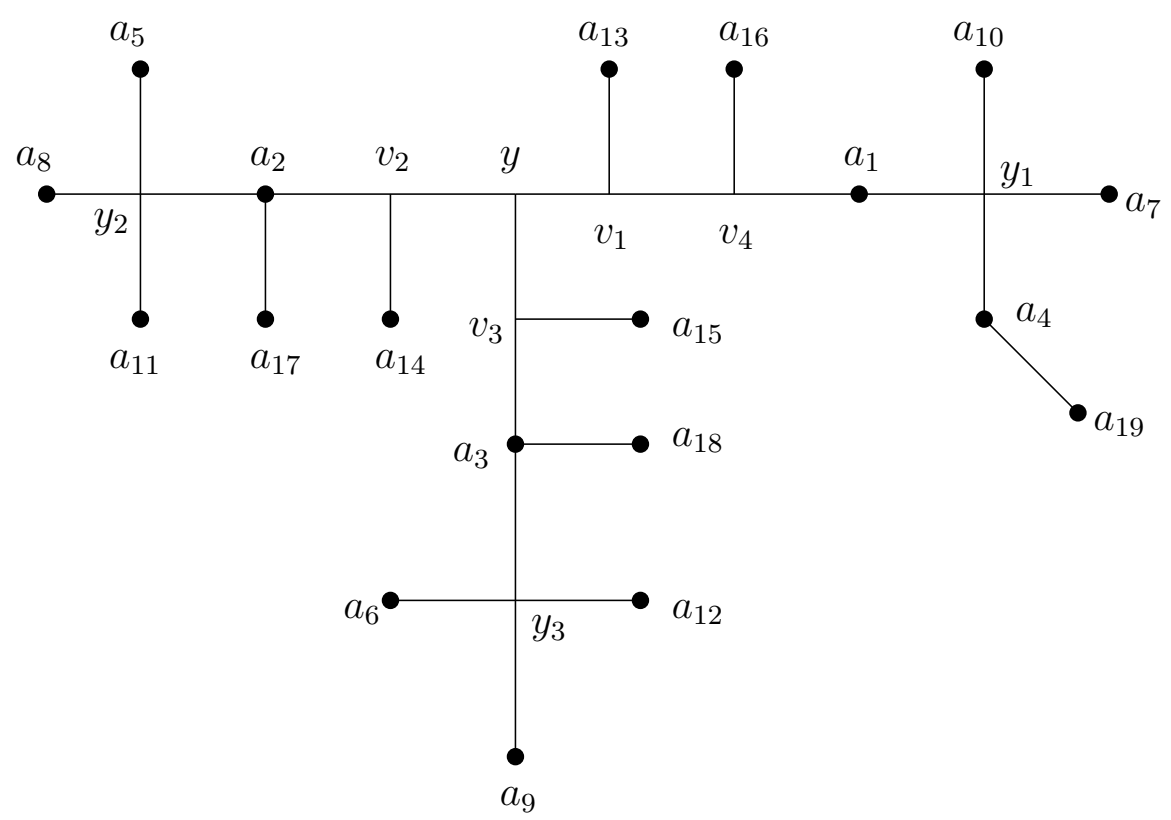

Fig. 7. A (19,3,2)-model

are left to the reader (see Fig. 6 for an example with $r=11, p=3$ and $q=4$, for which $s=0$ ). Under this assumption, we choose $T$ and a point $y \in T$ such that there are $p y$-branches and:

(i) $\mathrm{Cl}\left(Z^{\star}(A)\right)$ has $2 p+q-1$ endpoints.

(ii) For $1 \leq i \leq p, Z(A)_{i}$ contains an $(s+1)$-star whose endpoints are $a_{i}, a_{i+p}, \ldots, a_{i+s p}$. When $s>1$, we denote the central point of this star by $y_{i}$.

(iii) $Z^{\star}(A) \backslash\{y\}$ contains $p+q-1$ vertices of $T$, which we call $v_{1}, v_{2}, \ldots, v_{p+q-1}$.

(iv) Set $t=q \bmod p$. Now we define $r+p+q-t$ $A^{y}$-basic intervals, which are also edges of $T$ :

$$
\begin{gathered}
I_{i}=\left[y, v_{i}\right] \text { for } 1 \leq i \leq p, \\
I_{p+i}=\left[v_{i}, v_{p+i}\right] \text { for } 1 \leq i \leq q-1, \\
I_{p+q+i}=\left[v_{q+i}, a_{t+i}\right] \text { for } 0 \leq i \leq p-t, \\
I_{2 p+q-t+i}=\left[y_{i} \bmod p, a_{p+i}\right] \\
\text { for } 1 \leq i \leq s p, \\
I_{2 p+q-t+s p+i}=\left[v_{i}, a_{p+s p+i}\right] \\
\text { for } 1 \leq i \leq p+q-1 \text { and } \\
I_{3 p+2 q-t+s p+i}=\left[a_{2 p+s p+q+i}, a_{t+i}\right] \\
\text { for } 0 \leq i \leq k .
\end{gathered}
$$

Finally, $T$ contains also the following edges: $K_{i}=$ $\left[a_{i}, y_{i}\right]$ for $1 \leq i \leq p$ (recall that $K_{i}$ is not defined when $s<2)$ and $\left[v_{i}, a_{i \bmod p}\right]$ for $p+q-t+1 \leq i \leq$ $p+q-1$. See Fig. 7 for an example with $r=19$, $p=3$ and $q=2$.

Images of the vertices. We define $f(y)=y$, $f\left(v_{i}\right)=v_{i+1}$ for each $1 \leq i<p+q-1, f\left(v_{p+q-1}\right)=$ $a_{t}$ and $f\left(y_{i}\right)=y_{i+1} \bmod p$ for each $1 \leq i \leq p$.

(a) There is a unique periodic orbit $P=\left\{y_{i}\right\}_{i=1}^{p}$ of vertices in $T \backslash\{y\}$. Moreover, for each $A^{y}$-basic interval $\left[v, v^{\prime}\right]$ such that $v \in P$ we have that $v^{\prime} \in$ $A$. Thus there are no $f$-identifiable vertices and $(T, A, f)$ is a canonical model.

(b) Observe that $a_{i} \in Z\left(A^{y}\right)_{i} \bmod p$ for each $1 \leq$ $i \leq r$. In particular, the edge $I_{r+p+q-t}=\left[a_{r}, a_{t+k}\right]$ is contained in the $y$-branch $Z\left(A^{y}\right)_{r} \bmod p$, which is different from $Z_{p}$ since, by assumption, $r \notin p \mathbb{N}$. Then $f\left(a_{t+k}\right) \in Z\left(A^{y}\right)_{r+1} \bmod p \neq Z\left(A^{y}\right)_{1}$ and $f\left(a_{r}\right)=a_{1} \in Z\left(A^{y}\right)_{1}$. In consequence, $(T, A, f)$ is non-twist around $y$. Moreover, since the orbit of each vertex of $Z^{\star}(A) \backslash\{y\}$ contains $a_{t} \in A$, it follows that $(T, A, f)$ is $y$-expansive.

(c) Since $x\left(A^{y}\right)_{i}=v_{i}$ and $f\left(v_{i}\right)=v_{i+1}$ for $1 \leq$ $i \leq p$, we get that $p$ is a type of $A^{y}$. Furthermore, $\min \left\{q_{1}, q_{2}, \ldots, q_{p}\right\}=q_{p}$. Since $\left[y, f^{i}\left(v_{p}\right)\right] \cap A=$ $\left[y, v_{p+i}\right] \cap A=\emptyset$ for $1 \leq i \leq q-1$ and $\left[y, f\left(v_{p+q-1}\right)\right] \cap$ 
$A=\left\{a_{t}\right\}$, it follows that $q$ is the rotation index of the type $p$.

(d) The $f$-graph of $A^{y}$ contains exactly the following elementary loops:

(i) $I_{1} \rightarrow I_{2} \rightarrow \ldots \rightarrow I_{p} \rightarrow I_{1}$, which we denote by $\alpha$ and we take as the typical loop.

(ii) $I_{i} \rightarrow I_{i+1} \rightarrow \ldots \rightarrow I_{r+p+q-t} \rightarrow I_{i}$ for each $1 \leq i \leq 2 p+q-t$ such that $i \equiv 1(\bmod p)$, which we denote by $\beta_{i}$. Each $\beta_{i}$ is positive and $\left|\beta_{i}\right|=r+p+q-t-i+1 \in r+p \mathbb{N} \cup\{r\}$. Note that $\beta_{i}$ is external only when $i=1$.

(iii) $I_{i} \rightarrow I_{i+1} \rightarrow \ldots \rightarrow I_{r+p+q-t} \rightarrow I_{i}$ for each $1 \leq i<3 p+q-t$ such that $i \equiv r+1(\bmod p)$, which we denote by $\gamma_{i}$. Each $\gamma_{i}$ is negative and $\left|\gamma_{i}\right|=r+p+q-t-i+1 \in p \mathbb{N}$. The maximum of the lengths of $\gamma_{i}$ is $\left|\gamma_{r+1} \bmod p\right|=$ $r+p+q-t-(r \bmod p)$ and the minimum is $r-(r \bmod p)-p$. Note that $\gamma_{i}$ is external only when $i=r+1 \bmod p$.

(iv) $K_{1} \rightarrow K_{2} \rightarrow \ldots \rightarrow K_{p} \rightarrow I_{2 p+q-t+1} \rightarrow \ldots \rightarrow$ $I_{2 p+q-t+s p} \rightarrow K_{1}$, which we denote by $\delta$. Note that $\delta$ is negative and $|\delta|=(s+1) p$.

(v) $K_{r+1} \bmod p \rightarrow K_{r+1} \bmod p+1 \rightarrow \ldots \rightarrow K_{p} \rightarrow$ $I_{2 p+q-t+1} \rightarrow \ldots \rightarrow I_{r+p+q-t} \rightarrow K_{r+1 \bmod p}$, which we denote by $\epsilon$. Note that $\epsilon$ is positive and $|\epsilon|=r+1-(r+1 \bmod p) \in p \mathbb{N}$.

(vi) $I_{i} \rightarrow I_{i+1} \rightarrow \ldots \rightarrow I_{2 p+q-t+s p} \rightarrow I_{i}$ for each $p+1 \leq i<2 p+q-t$ such that $i \equiv 1(\bmod$ $p$ ), which we denote by $\sigma_{i}$. Note that $\sigma_{i}$ is negative and $\left|\sigma_{i}\right|=2 p+q-t+s p-i+1 \in p \mathbb{N}$.

(vii) $I_{2 p+q-t} \rightarrow K_{1} \rightarrow K_{2} \rightarrow \ldots \rightarrow K_{p} \rightarrow$ $I_{2 p+q-t+1} \rightarrow \ldots \rightarrow I_{r+p+q-t} \rightarrow I_{1} \rightarrow \ldots \rightarrow$ $I_{2 p+q-t}$, which we denote by $\tau$. Note that $\tau$ is negative and $|\tau|=r+2 p+q-t \in r+p \mathbb{N}$.

Since $p+q-t=l p$, we have $\left|\beta_{1}\right|=r+l p$. By Lemma 3.3 , there is a fixed point $x \in I_{1}=\left[y, v_{1}\right]$ of $f^{r+l p}$ associated to $\beta_{1}$. Since $v_{1}$ is not periodic, $x \neq v_{1}$. Moreover, $x \neq y$ since $y \in \operatorname{Fix}(f)$ and some iterate of $x$ belongs to $I_{r+l p}$, which does not contain $y$. Therefore, $x \in\left(y, v_{1}\right)$. Since $\beta_{1}$ is simple, from Lemma 3.5 it follows that the period of $x$ is $r+l p$. On the other hand, $y \in \operatorname{Fix}(f)$ and $p \in \operatorname{Per}(f)$ by Lemma 4.2. Therefore,

$$
\operatorname{Per}(f) \supset\{1, p, r+l p\} .
$$

Since $\beta_{1}$ and $\gamma_{r+1 \bmod p}$ are external, by Lemma 4.6 we get $\operatorname{Per}(g) \supset\{(r+l p) i+p j: i, j \geq 1\} \cup\{(r-$ $(r \bmod p)+l p) i+p j: i, j \geq 1\} . \quad$ Furthermore, since each $\gamma_{i}$ is simple and negative it satisfies (b) of Lemma 3.5 and, hence, $f$ has periodic orbits of periods $\{r-(r \bmod p)-p, r-(r \bmod p), \ldots, r-$ $(r \bmod p)+l p\}$. So we have

$$
\begin{aligned}
\operatorname{Per}(f) \supset\{(r+l p) i+p j: i, j \geq 1\} \cup \\
p \mathbb{N} \backslash\{2 p, 3 p, \ldots, r-(r \bmod p)-2 p\} .
\end{aligned}
$$

From this and (23) it follows that

$$
\begin{aligned}
\operatorname{Per}(f) \supset & \mathcal{S}_{p}^{*}(r+l p) \backslash \\
& \{2 p, 3 p, \ldots, r-(r \bmod p)-2 p\} .
\end{aligned}
$$

Since $\lambda p$ is defined to be the larger multiple of $p$ smaller than $r+p+q+1$, we have $\lambda p>r-(r \bmod$ $p)-2 p$ and from (24) we get

$$
\operatorname{Per}(f) \supset \mathcal{S}_{p}^{*}(r+l p) \backslash\{2 p, 3 p, \ldots, \lambda p\} .
$$

To end the proof we must show that $\operatorname{Per}(f) \subset$ $\mathcal{S}_{p}^{*}(r)$. The only periodic orbits contained in $A^{y}$ are $\{y\}, A$ and $\left\{y_{1}, y_{2}, \ldots, y_{p}\right\}$. On the other hand, by Lemma 3.2 each periodic orbit which does not intersect $A^{y}$ is associated to a loop. Each loop in the $f$ graph of $A^{y}$ is either elementary or a concatenation of some elementary loops, which are $\alpha, \beta_{i}, \gamma_{i}, \delta, \epsilon$, $\sigma_{i}, \tau$ and their respective shifts. The length of each of these elementary loops belongs to $p \mathbb{N} \cup(r+p \mathbb{N})$. Given $i, j \in \mathbb{N}$ and $m, n \in p \mathbb{N} \cup(r+p \mathbb{N})$, we have $i m+j n \in \mathcal{S}_{p}^{*}(r)$. It follows that the length of each loop in the $f$-graph of $A^{y}$ belongs to $\mathcal{S}_{p}^{*}(r)$ and thus (d) holds.

Case 4. $p>1, q>0$ and $r \in p \mathbb{N}$.

The construction is very similar to that of the previous case. We have $a_{i} \in Z\left(A^{y}\right)_{i \bmod p}$ for $1 \leq i<r$, but in this case we replace the edge $\left[a_{r}, a_{t+k}\right]$ by an edge $\left[y, a_{r}\right]$ contained in $\mathrm{Cl}\left(Z^{*}(A)\right)$. The details are left to the reader. See Fig. 8 for an example with $r=20, p=4$ and $q=1$.

Proof of Theorem 9.1. In order to prove (a) we use Proposition 9.2 iteratively. Set $k_{i}=p_{1} p_{2} \cdots p_{i}$ with $p_{i}>1$ for $1 \leq i \leq m$. Let us consider a tree $R_{0}$ consisting of a single point $x$ and the map $h_{0}(x)=x$. Then $\left(R_{0},\{x\}, h_{0}\right)$ is a canonical model such that $\operatorname{Per}\left(h_{0}\right)=\{1\}$. We use Proposition 9.2 with $s=p_{m}$ and obtain a canonical model $\left(R_{1}, B_{1}, h_{1}\right)$ such that $\operatorname{Per}\left(h_{1}\right)=\{1\} \cup p_{m} \operatorname{Per}\left(h_{0}\right)=\left\{1, p_{m}\right\}$ and $\left|B_{1}\right|=$ 


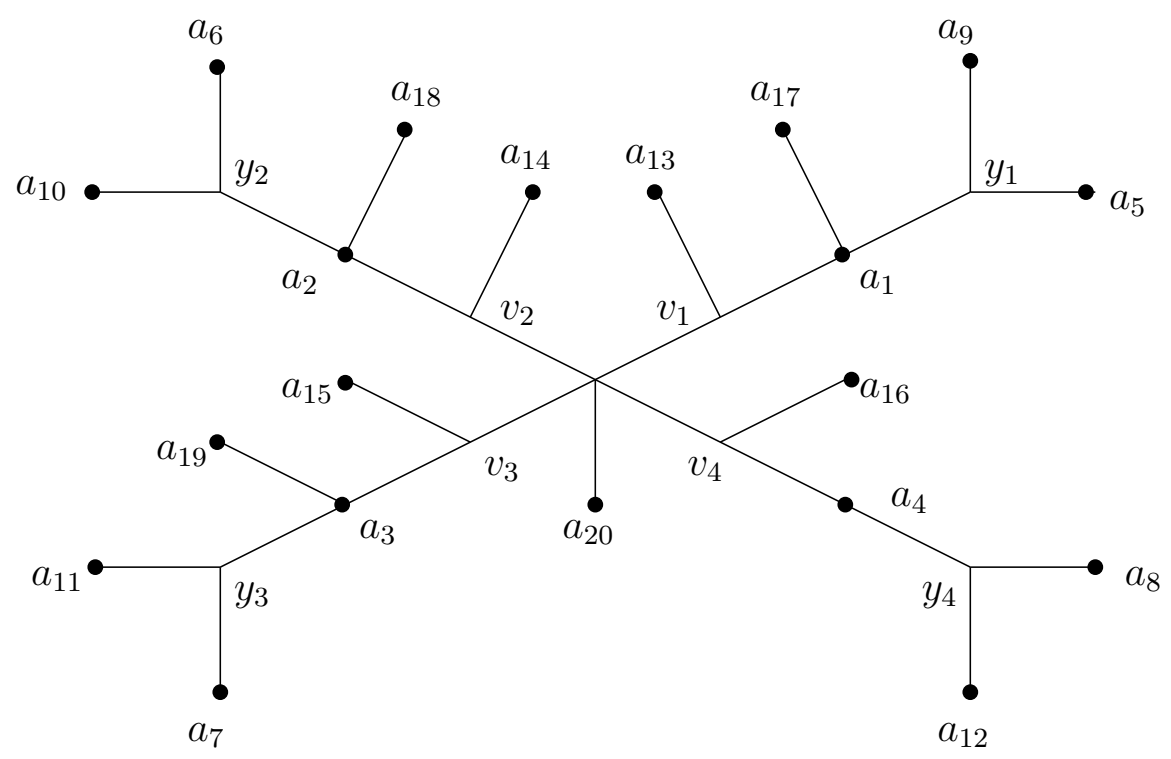

Fig. 8. A (20,4,1)-model

$p_{m}$. We use again Proposition 9.2 with $s=p_{m-1}$ and obtain a canonical model $\left(R_{2}, B_{2}, h_{2}\right)$ such that $\operatorname{Per}\left(h_{2}\right)=\{1\} \cup p_{m-1} \operatorname{Per}\left(h_{1}\right)=\left\{1, p_{m-1}, p_{m-1} p_{m}\right\}$ and $\left|B_{2}\right|=p_{m} p_{m-1}$. We can iterate this argument $m-1$ times and finally obtain a canonical model $\left(R_{m-1}, B_{m-1}, h_{m-1}\right)$, which we denote as $(R, B, h)$. Then $|B|=p_{1} p_{2} \cdots p_{m-1}$ and $\operatorname{Per}(h)=$ $\left\{p_{1}, p_{1} p_{2}, \ldots, p_{1} p_{2} \cdots p_{m}\right\}=K$. Thus (a) is proved.

Now we prove (b) analogously. We must construct a canonical model $(S, P, g)$ with all the prescribed properties. By Proposition 9.3, we can consider an $(r, p, q)$-model, which we denote by $\left(T_{m}, A_{m}, f_{m}\right)$. Since $A_{m}$ is a periodic orbit, $\left(T_{m}, A_{m}, f_{m}\right)$ is 0 -orbital. As above, we use $m-1$ times Proposition 9.2 to obtain a sequence of canonical models $\left(T_{i}, A_{i}, f_{i}\right)$ for $1 \leq i<m$ such that

(i) For $1 \leq i<m$ there exists $y_{i} \in \operatorname{Fix}\left(f_{i}\right)$ such that $\left(T_{i}, A_{i}, f_{i}\right)$ is a $y_{i}$-expansive 0 -orbital canonical model.

(ii) $\left(T_{i+1}, A_{i+1}, f_{i+1}\right)$ is a partial $p_{i}$-reduction of $\left(T_{i}, A_{i}, f_{i}\right)$ for each $1 \leq i<m$. Furthermore, $\operatorname{Per}\left(f_{i}\right)=\{1\} \cup p_{i} \operatorname{Per}\left(f_{i+1}\right)$.

(iii) $\left|A_{i}\right|=p_{i}\left|A_{i+1}\right|$ for $1 \leq i<m$.

(iv) $\left(T_{i}, A_{i}, f_{i}\right)$ is twist around $y_{i}$ for $1 \leq i<m$. Thus, by Remark 6.1, $\mathcal{E}\left(T_{i}, A_{i}, f_{i}\right)=\emptyset$. In particular, $\tilde{\mathcal{E}}\left(T_{i}, A_{i}, f_{i}\right)=\emptyset$.

By Proposition 9.3(a-c), $\left|A_{m}\right|=r$, there exists $y_{m} \in \operatorname{Fix}\left(f_{m}\right)$ such that $\left(T_{m}, A_{m}, f_{m}\right)$ is non-twist around $y_{m}$ and $p$ is a type of $A_{m}^{y_{m}}$. Furthermore, since $\operatorname{En}\left(T_{m}\right) \subset A_{m}$, from Proposition 6.5 we get $\tilde{\mathcal{E}}\left(T_{m}, A_{m}, f_{m}\right) \neq \emptyset$.

So if we set $p_{m}=p$ then $\left\{\left(T_{i}, A_{i}, f_{i}\right), y_{i}, p_{i}\right\}_{i=1}^{m}$ is a sequence of partial reductions of $\left(T_{1}, A_{1}, f_{1}\right)$. Therefore, if we define $(S, P, g)=\left(T_{1}, A_{1}, f_{1}\right)$ and $(\bar{S}, \bar{P}, \bar{g})=\left(T_{m}, A_{m}, f_{m}\right)$ then $\{(\bar{S}, \bar{P}, \bar{g}), K\}$ is a complete reduction of $(S, P, g)$.

From the properties of an $(r, p, q)$-model listed in Proposition 9.3 we get that $|\bar{P}|=\left|\bar{P}^{\circ}\right|=r$ and $q$ is a rotation index associated to the type $p$. It follows that $p(\bar{S}, \bar{P}, \bar{g})=p$ and $q(\bar{S}, \bar{P}, \bar{g})=q$. Moreover, $n(\bar{S}, \bar{P}, \bar{g})=0$ since $(\bar{S}, \bar{P}, \bar{g})$ is 0-orbital. Finally,

$$
\begin{gathered}
\mathcal{S}_{p}^{*}(r+l p) \backslash\{2 p, 3 p, \ldots, \lambda p\} \\
\subset \operatorname{Per}(\bar{g}) \subset \mathcal{S}_{p}^{*}(r),
\end{gathered}
$$

where $l p=p+q-(q \bmod p)$ and $\lambda p$ is the largest multiple of $p$ smaller than $r+p+q+1$. From (ii) it follows that

$$
\operatorname{Per}(g)=K \cup k \operatorname{Per}(\bar{g})
$$

By Remark 2.2, the theorem follows from (25) and (26) by taking $\mathcal{C}=k \operatorname{Per}(\bar{g})$.

\section{Acknowledgements}

All the authors have been partially supported by the DGES grant number PB96-1153. The first author has been partially supported by the CONACIT 
grant number 1999SGR-00349 and the third author by CIRIT grant number 2000SGR-00027.

\section{References}

Alsedà, Ll., Guaschi, J., Los, J., Mañosas, F., Mumbrú, P. [1997] "Canonical representatives for patterns of tree maps", Topology 36, 11231153.

Alsedà, Ll., Juher, D., Mumbrú, P. [2001] "A note on the periodic orbits and topological entropy of graph maps", Proc. Amer. Math. Soc. 129, no. 10, 2941-2946.

Alsedà, Ll., Llibre, J., Misiurewicz, M. [1989] "Periodic orbits of maps of Y", Trans. Amer. Math. Soc. 313, 475-538.

Alsedà, Ll., Llibre, J., Misiurewicz, M. [2000] Combinatorial dynamics and entropy in dimension one, Advanced Series in Nonlinear Dynamics 5, World Scientific, second edition.

Alsedà, Ll., Ye, X. [1995] "No division and the set of periods for tree maps", Ergod. Th. \& Dynam. Sys. 15, 221-237.

Baldwin, S. [1987] "Generalizations of a theorem of Sharkovskii on orbits of continuous real-valued functions", Discrete Math. 67, 111-127.

Baldwin, S. [1991] "An extension of Sharkovskii's Theorem to the n-od", Ergod. Th. \&5 Dynam. Sys. 11, 249-271.

Block, L. [1981] "Periods of periodic points of maps of the circle which have a fixed point", Proc. Amer. Math. Soc. 82, 481-486.

Block, L., Guckenheimer, J., Misiurewicz, M., Young, L.S. [1980] "Periodic points and topological entropy of one-dimensional maps", Global theory of dynamical systems, pp. 18-34, SLNM 819, Springer, Berlin.

Blokh, A.M [1991] "On some properties of graph maps: spectral decomposition, Misiurewicz conjecture and abstract sets of periods", preprint, Max Planck Institut für Mathematik, Bonn.

Blokh, A.M [1992] "Periods implying almost all periods for tree maps", Nonlinearity 5, 1375-1382.
Denker, M., Grillenberger, C., Sigmund, K. [1976] Ergodic theory on compact spaces, SLNM 527, Springer, Berlin.

Efremova, L.S. [1978] "Periodic orbits and a degree of a continuous map of a circle" (in Russian), Diff. and Integr. Equations (Gor'kii) 2, 109-115.

Imrich, W., Kalinowski, R. [1985 a] "Periodic points of small periods of continuous mappings of trees", Ann. Discrete Math. 27, 443-446.

Imrich, W., Kalinowski, R. [1985 b] "Periodic points of continuous mappings of trees", Ann. Discrete Math. 27, 447-460.

Li, T.-Y., Misiurewicz, M., Pianigiani, G., Yorke, J.A [1982] "No division implies chaos", Trans. Amer. Math. Soc. 273 191-199.

Llibre, J., Misiurewicz, M. [1993] "Horseshoes, entropy and periods for graph maps", Topology 32, 649-664.

Misiurewicz, M [1982] "Periodic points of maps of degree one of a circle", Ergod. Th. \&5 Dynam. Sys. 2, 221-227.

Misiurewicz, M., Nitecki, Z. [1991] "Combinatorial patterns for maps of the interval", Mem. Amer. Math. Soc. 94, no. 456

Sharkovskii, A.N. [1964] "Co-existence of the cycles of a continuous mapping of the line into itself" (in russian), Ukrain. Math. Zh. 16 (1), 61-71. English translation in Proceedings of the Conference "Thirty Years after Sharkovskii's Theorem: New Perspectives" (Murcia, 1994), Internat. J. Bifur. Chaos Appl. Sci. Engrg. 5 (1995), 12631273. 